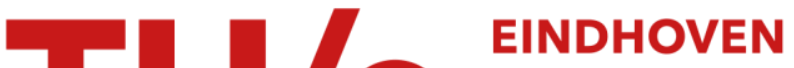

\section{Strong normalization in a typed lambda calculus with lambda structured types}

\section{Citation for published version (APA):}

Nederpelt, R. P. (1973). Strong normalization in a typed lambda calculus with lambda structured types. [Phd Thesis 1 (Research TU/e / Graduation TU/e), Mathematics and Computer Science]. Technische Hogeschool Eindhoven. https://doi.org/10.6100/IR145802

DOI:

10.6100/IR145802

Document status and date:

Published: 01/01/1973

\section{Document Version:}

Publisher's PDF, also known as Version of Record (includes final page, issue and volume numbers)

\section{Please check the document version of this publication:}

- A submitted manuscript is the version of the article upon submission and before peer-review. There can be important differences between the submitted version and the official published version of record. People interested in the research are advised to contact the author for the final version of the publication, or visit the $\mathrm{DOI}$ to the publisher's website.

- The final author version and the galley proof are versions of the publication after peer review.

- The final published version features the final layout of the paper including the volume, issue and page numbers.

Link to publication

\section{General rights}

Copyright and moral rights for the publications made accessible in the public portal are retained by the authors and/or other copyright owners and it is a condition of accessing publications that users recognise and abide by the legal requirements associated with these rights.

- Users may download and print one copy of any publication from the public portal for the purpose of private study or research.

- You may not further distribute the material or use it for any profit-making activity or commercial gain

- You may freely distribute the URL identifying the publication in the public portal.

If the publication is distributed under the terms of Article $25 \mathrm{fa}$ of the Dutch Copyright Act, indicated by the "Taverne" license above, please follow below link for the End User Agreement:

www.tue.nl/taverne

Take down policy

If you believe that this document breaches copyright please contact us at:

openaccess@tue.nl

providing details and we will investigate your claim. 


\section{STRONG NORMALIZATION IN}

\section{A TYPED LAMBDA CALCULUS WITH LAMBDA STRUCTURED TYPES}


STRONG NORMALIZATION IN

A TYPED LAMBDA-CALCULUS WITH

LAMBDA-STRUCTURED TYPES 


\title{
STRONG NORMALIZATION IN A TYPED LAMBDA CALCULUS WITH LAMBDA STRUCTURED TYPES
}

\author{
PROEFSCHRIFT
}

TER VERKRIJGING VAN DE GRAAD VAN DOCTOR IN DE TECHNISCHE WETENSCHAPPEN AAN DE TECHNISCHE HOGESCHOOL EINDHOVEN, OP GEZAG VAN DE RECTOR MAGNIFICUS, PROF, DR, IR, G, VOSSERS, VOOR EEN COMMISSIE AANGEWEZEN DOOR HET COLLEGE VAN DEKANEN IN HET OPENBAAR TE VERDEDIGEN OP DINSDAG 12 JUNI 1973 TE 16,00 UUR

DOOR

ROBERT PIETER NEDERPELT LAZAROM

GEBOREN TE 'S-GRAVENHAGE

C 1973 by R.P. Nederpelt, Eindhoven, The Netherlands 
DIT PROEFSCHRIFT IS GOEDGEKEURD

DOOR DE PROMOTOREN

PROF.DR. N.G. DE BRUIJN

en

PROF.DR. W. PEREMANS 
CONTENTS

Conventions as regards references

VII

CHAPTER I. Introduction and summary

\$1. Lambda-calculus

\$ 2. Normalization and strong normalization 3

5 3. Normalization in systems of typed lambda-calculus 5

\$ 4. The relation to the mathematical language Automath 8

5 5. Change of notational conventions 11

$\$ 6$. Summary of the contents of this thesis 12

CHAPTER II. The formal system $\Delta$

\$ 1. Alphabet and syntactical variables 17

\$ 2. Expressions 19

5 3. Bound expressions 23

5 4. Replacement, renovation and $\alpha$-reduction 29

\$ 5. Substitution and $\beta$-reduction 33

\$ 6. Other B-reductions 42

\$ 7. n-reduction, reduction and lambda-equivalence 60

\$ 8. Type and degree $\quad 72$

CHAPTER III. The formal system $\Lambda$

\$ 1. Legitimate expressions 76

\$ 2. The normalization theorem 81

\$ 3. Strong normalization 90

$\begin{array}{ll}\text { References } & 97\end{array}$

$\begin{array}{ll}\text { Samenvatting } & 100\end{array}$

$\begin{array}{ll}\text { Curriculum Vitae } & 102\end{array}$ 
CONVENTIONS AS REGARDS REEERENCES

References to literature are denoted by the name(s) of the author(s) and a number in square brackets which is sometimes followed by a further indication (e.g. Church $[2, \mathrm{p}, 36]$ ). These references are listed at the end of this thesis.

This thesis contains three chapters, which are denoted by capital Roman numerals. Each chapter consists of a number of sections. In referring, for example, to \& 3 of Chapter II, we write section II.3. In each section we number definitions, theorems and notation rules consecutively, indicating the section number in front of the serial number. Thus, in Section II. 3 we may propose Theorem 3.5. A reference to this theorem in the pertaining chapter is written as Th. 3.5 , in other chapters as Th. II.3.5. 


\section{$\S 1$. LAMBDA-CALCULUS}

The lambda-notation was originally introduced as a useful notation by Church in two papers developing a system of formal logic [2]. He extended this notation in his calculus of lambdaconversion (Zambda-calculus). This calculus was meant to describe a general class of functions which have the feature that they can be applied to functions of this same class.

For historical comment see Curry and Feys [3, Ch. $0, \S \mathrm{D}$ and Ch. 3, $\S \mathrm{S} 1]$ and Barendregt $[1, \mathrm{Ch}, 1, \S 1.1]$. In the latter reference the importance of lambda-calculus for the development of recursive functions is mentioned. The calculus has also been brought into relation with the theory of ordinal numbers, predicate calculus and other theories. From the very beginning, lambda-calculus was strongly linked to the theory of combinatory logic.

We shall later mention some major results achieved concerning lambda-calculus. Right here we stress the contribution of lambdacalculus to ordinary mathematics at a purely notational level. The mathematical custom to use the notation $f(x)$, both for the function itself and for the value of this function at an undetermined argument $x$, obscures the mathematical notion "function". According to Curry and Feys "this defect is especially striking in theories which employ functional operations (functions which admit other functions as arguments)". For an example showing that the usual mathematical function notation is defective not only for understanding, but also in use, see Curry and Feys [3, Ch. $3, \S$ A2].

We shall give an example of the lambda-notation. Consider the function which assigns to $x$ the value $x+2$. This function is denoted in lambda-notation as $\lambda x \cdot x+2$. We can apply the function to an argument, say 3 . The application of this function to the argument 3 is denoted as $(\lambda x \cdot x+2) 3$. The result of this application must clear$1 y$ be $3+2$. 
This suggests that there exists an order between the terms $(\lambda x \cdot x+2) 3$ and $3+2$ (the latter term is "closer to the outcome"). The transitive and reflexive relation corresponding to such an order is called a reduction. In the above case it is called a Breduction, often denoted by $z_{\beta}$. Thus we have the relation $(\lambda x \cdot x+2) 3 \geq_{B} 3+2$.

The reduction relation is also monotonous, i.e.: if term $S$ reduces to term $T$, then $\lambda x \cdot S$ reduces to $\lambda x \cdot T,(U) S$ to (U) T and (S)U to (T)U. So from the relation $(\lambda x \cdot x+2) 3 \geq_{B} 3+2$ follows, for example, that $\lambda y \cdot((\lambda x \cdot x+2) 3) \geq_{\beta} \lambda y \cdot(3+2)$.

The relation compares two terms (viz. $(\lambda x * x+2) 3$ and $3+2)$; the fact that these terms have the common value 5 in the usual interpretation, plays no rôle here.

If we do not take 3 , but $x$ as argument for the above function, then we obtain $(\lambda x \cdot x+2) x \geq_{\beta} x+2$. So lambda-calculus makes a clear distinction between the function: $\lambda x \cdot x+2$ and the value of this function for an undetermined argument: $x+2$.

We are used to the fact that the terms $\lambda x * x+2$ and $\lambda y \cdot y+2 d e-$ note the same function. The two terms are called $\alpha$-equivazent, and the passage of the one into the other is called $\alpha$-reduction, often denoted by $\geq_{\alpha}$. In this way we also have the relation $\lambda x \cdot x+2 \geq_{\alpha} \lambda y \cdot y+2$. It is quite a nuisance that this a-reduction, which is simply a renaming of variables, plays a rôle in the lambda-notation. One can avoid this by considering $\alpha$-equivalence classes instead of separate terms. Another nice and practical way out is given by De Bruijn [8], who completely suppresses the use of names of variables by means of a notational system referring to the positions of a variable in a term.

We wish to state that the desire to eliminate variables is one of the things giving rise to combinatory logic. The method used in combinatory logic to obtain this elimination is, however, different from De Bruijn's.

A third reduction, which is comonly used and strongly related to extensionality (see Barendregt $[1, \mathrm{Th}, 1,1.17$ and $\mathrm{Th}, 1,1.18]$ ), 
is called $n$-reduction. This relation, commonly denoted by $\geq \eta^{\prime}$ is based on the following rule: If $x$ is not free in the term $M$, then $\lambda x \cdot(M) x \geq_{\eta} M$. An intuitive justification is that, for any argument $X$, the sides of the relation have comparable values: this value is $(\lambda \mathrm{x} \cdot(M) \mathrm{x}) \mathrm{X}$ for the left-hand side and $(M) \mathrm{X}$ for the right-hand side, and $(\lambda x \cdot(M) x) x \geq_{B}(M) X$.

A sequence of reductions obtained by successive application of reductions is called a reduction sequence.

For each of the reduction relations explained above, the corresponding symmetric and transitive closure is called a conversion relation. One of the first important results in lambda-calculus concerns the dependence between conversion and reduction. This is called the Church-Rosser theorem, which states: If $X$ converts to $Y$, then there is a $Z$ such that $X$ reduces to $Z$ and $Y$ reduces to $Z$ (see Curry and Feys $[3, \mathrm{Ch} .4])$. For interesting historical comments see Barendregt [1, Th, 1.2 .9 and the remarks in 1.2 .18 plus footnote]. In Appendix II of the latter reference the latest and nicest proof of the Church-Rosser theorem is given (1971 by W.W. Tait and P. Martin-Löf). For a precise description see Schulte Mönting [24].

In this thesis we shall use the name "Church-Rosser property" for the following statement: If $A$ reduces to $B$ and to $C$, then there is a $D$ such that $B$ and $C$ reduce to $D$. This property is equivalent to the Church-Rosser theorem.

\section{2. NORMALIZATION AND STRONG NORMALIZATION}

An important issue in lambda-calculus is the question of the normalization of terms. This is a termination problem. For example, a $\beta$-reduction such as $(\lambda x \cdot x) y \geq_{\beta} y$ cannot be continued in a nontrivial manner: there is no reduction for $y$, except those trivial on account of the reflexivity of $\alpha-, \beta-$ and $n$-reduction. In this case $(\lambda x \cdot x) y$ is said to normalize into a normal form $y$. 
In lambda-calculus, which allows all functions as arguments of functions, such a termination of the reduction is not guaranteed. See Church's nice example: $\omega_{2}=(\lambda x \cdot x x)(\lambda x \cdot x x)$. There is a nontrivial $\beta$-reduction, by applying the rule $(\lambda x \cdot x x) A \geq_{B}$ (A)A with $A=\lambda x \cdot x x$. This produces $\omega_{2} \geq_{\beta} \omega_{2}$. It is clear that the reduction of $\omega_{2}$ by repeated use of the above non-trivial B-reduction will never come to an end.

There are more and stranger examples of such terms, the reduction of which never terminates. For example: put $\omega_{3}=\lambda x \cdot x x x$. Then $\omega_{3} \omega_{3} \geq_{8} \omega_{3} \omega_{3} \omega_{3} \geq_{B} \ldots$. Barendregt even constructed a universal generator with the property that it has a reduction sequence in which all terms of lambda-calculus occur as subterms.

A term in lambda-calculus is called nomalizable if there is some reduction sequence which terminates. A term is strongly normalizable if each of its reduction sequences terminates. The last term of a terminating sequence is called a nomal form.

It is obvious that strong normalization implies normalization. The reverse implication does not hold. For example: put again $\omega_{2}=\lambda x \cdot x x$; then $(\lambda x \cdot(\lambda y \cdot y))\left(\omega_{2} \omega_{2}\right)$ reduces to $\lambda y \cdot y$ if the function $\lambda x \cdot(\lambda y \cdot y)$ is applied to the argument $\omega_{2} \omega_{2}$, but it reduces to itself if the function $\omega_{2}$ is applied to the argument $\omega_{2}$. Since $\lambda y \cdot y$ is in normal form, $(\lambda x \cdot(\lambda y \cdot y))\left(\omega_{2} \omega_{2}\right)$ is normalizable, but not strongly normalizable.

In this example we see a term that normalizes if one application of a function to an argument is assigned priority over another. There is a general theorem in lambda-calculus (the standardization theorem, cf. Curry and Feys [3, Ch. 4E1]), which states that any normalizable term can be normalized by assigning priority to the "leftmost" application in the term.

The fact that some term in lambda-calculus have non-terminating reduction sequences is related to the feature that one can use al1 functions as arguments for functions. (Even the function itself can be used as an argument, see the above-mentioned example by Church. This is called self-application.) 
The same things can happen in programming languages and in the theory of partial recursive functions, where normalization- (or termination-) problems arise too.

In lambda-calculus the question of the normalizability of terms has been shown to be undecidable.

There are systems in which normalization implies strong normalization. For example, in a restricted lambda-calculus ( $\lambda I-c a l c u-$ lus) this implication holds (the so-called second Church-Rosser theorem, see Curry and Feys $[3, \mathrm{Ch}, 4,5 \mathrm{E}]$ ), but the proof is not trivial.

Prawitz [17] proved normalization for derivations in natural deduction. He also proved strong normalization for these derivations in [18]. Note that in the latter proof he does not use his results from [17], but quite a different proof technique developed by Tait [21].

An interesting problem concerning normalization is the question of uniqueness of normal forms. If a term A has the property that every terminating reduction sequence leads to the same normal form (but for $\alpha$-reduction), then $A$ is said to have a unique normal form. We note that the Church-Rosser theorem implies the uniqueness of the normal form if this exists.

In this thesis we shall show that, if in a system all terms are normalizable into a unique normal form, then each term is strongly normalizable. This will be proved for a certain lambdacalculus called $A$, the method can, however, be applied to more systems, and we suggest this as a field of further investigation.

\section{§ 3. NORMALIZATION IN SYSTEMS OF TYPED LAMBDA-CALCULUS}

In ordinary mathematics one, sometimes tacitly, assumes that each object has a certain type (in our example of a term in lambdanotation: $\lambda x \cdot x+2$, we assumed that $x$ has a type (e.g. that of the natural numbers) in which addition is possible). In systems of typed lambda-calculus one attaches a type to each term. In so doing 
and in restricting the formation of terms in accordance with the types (see the "applicability condition" explained in Section I.4) one brings lambda-calculus nearer to usual mathematical systems.

We note here that there is a strong correspondence between derivations in systems of natural deduction and terms in systems of typed lambda-calculus, as well as between formulae in the one and types in the other: a derivation $D$ proving a formula $F$ corresponds to a term $D^{\prime}$ with type $F^{\prime}$. This is called the "formulae-as-type notion".

The latter notion has recently been investigated by various authors in developing a theory of construction and in studying functional interpretations. The first indication in this direction was given in Curry and Feys $[3, p, 312-315]$. We further mention Läuchli [11], De Bruijn, who developed and applied this notion with a large variety of types in his mathematical language Automath ([4]), Howard [10], Prawitz [18] and Girard [9].

Normalization problems also arise in systems of typed lambdacalculus. Sanchis [19] investigated a lambda-calculus with types (essentially Gödel's theory of functionals of finite type) and found all terms in this calculus to be strongly normalizable.

Martin-Löf [12] admitted more general types and obtained normalization for his terms. His system is close to the requirements of common mathematics in the sense that usual mathematical notions such as the logical connectives and the recursion operator are incorporated.

In this thesis we shall regard a typed lambda-calculus, in which the types themselves have lambda-structure. Our typed lambdacalculus, which we call $\mathrm{A}$, has a large overlap with the mathematical language Automath [4]. (See the following section for the relation between Automath and our system $\Lambda_{\text {. }}$ )

In particular, a single-line version of Automath (AUT-SL, see [7]) introduced by De Bruijn has led us to the investigations in this thesis. Preliminary work in the direction of AUT-SL can be found in our notes on Lambda-Automath ([13] and [14]), in which 
some syntactical notions of Automath were unified. In AUT-SL this unification was extended considerably.

De Bruijn defined AUT-SL by means of a recursive programme. our definition of system $\Lambda$ (given in Chapter III) follows more orthodox recursive lines. Nevertheless, the resulting systems are the same.

In these systems there is no syntactical distinction between terms and types. We therefore use the word expression rather than term or type. There is one basic constant in the system, called $\tau$. To each expression which does not end in $\tau$ we shall assign a type in a natural manner.

We say that expressions ending in $\tau$ have degree 1. Each other expression has some degree $\mathfrak{n}>1$, while the degree of such an expression $A$ is defined to be one more than the degree of the type of A. In this manner we have expressions of any finite degree at our disposal.

In Automath and in Martin-Löf's system there is a restriction to the degrees permitted. Both systems have only terms and types of degree 1, 2 or 3.

Our system has in common with Automath that logical connectives, the recursion operator and a basic set of numbers (e.g. natural numbers) are not incorporated. The proofs of normalization results concerning these systems can be formalized in first order arithmetic.

Yet it is possible to interpret into these systems mathematical theories containing, for instance, logical connectives and the recursion operator by introducing new primitive equality relations which extend the existing equality relations which correspond to conversion.

We shall prove normalization and strong normalization for our system in Chapter III. As mentioned above, we shall introduce a method for deriving strong normalization from normalization together with the uniqueness of normal forms (see Section I.6). 
\$ 4. THE RELATION TO THE MATHEMATICAL LANGUAGE AUTOMATH

Automath (see [4] and [5]) was designed by De Bruijn as a language for mathematics. It has the property that the interpretation of a text written in Automath is correct mathematics if the text is syntactically correct.

Many such systems have been developed for logic. For mathematics, Russell and Whitehead's Principia Mathematica was the first successful attempt in the direction of formalization. There have since been many other attempts.

However, in the majority of these systems important parts of the mathematical argumentation were not incorporated in the formal system, but were dealt with at a meta-level. For example, in systems based on axioms and inference rules a theorem is true if it can be inferred by successive application of a number of axioms and rules. But one hardly ever says exactly (in terms of the formal language) which axioms and rules were used, and in which order. Moreover, the use of an axiom scheme was usually not substantiated by a formalized indication of the substitution instance employed. Admittedly, there is a gap in the completeness of the formalization in Automath, too. The gap is that, in the case of "definitionally equal" expressions, there is no indication of how this equality can be established on the basis of the language definition. It is left to algorithms to justify these definitional equalities.

The existence of terminating algorithms for this purpose can be proved by means of normalization properties. The question of practical efficiency of such algorithms is, of course, a different one, and is not considered in this thesis.

Two expressions in Automath are called definitionalzy equal if one expression can be transferred into the other by (1) conversions and (2) the elimination of abbreviations.

A major problem for automatic checking in Automath is whether definitional equality of two expressions is decidable. The latter is clearly the case if each expression is effectively normalizable into a unique normal form. In this respect, see Kreise1 [23]. 
The main aim of this thesis is to prove the existence and uniqueness of normal forms for $\Lambda$. Since $\Lambda$ does not use an abbreviation system as a syntactical element like Automath does, we may restrict ourselves to conversions. We note that the omission of abbreviations is no severe restriction, since abbreviations are relatively simple operations usually, considered to be only notational devices without mathematical content.

The mere typing of lambda-calculus expressions does not guarantee the property of normalization. We need more.

Automath permits only a restricted class of expressions. In this class only those expressions $E$ are included which obey the socalled applicability condition: for each part of $E$ which has the form of a function $F$ applied to an argument $A$ it is required that (1) $F$ has a domain $D$, and (2) the type of $A$ is definitionally equal to $D$.

These requirements are natural for a system which is so closely linked to ordinary mathematics. The following examples in lambdanotation will make this clear. In the first place, it would be unnatural to supply an expression which is not a function with an argument: one can attach an argument to $\lambda x * x+2$, but it looks strange to provide the number 7 with an argument.

Secondly, let us assume that $x$ in $\lambda x \cdot x+2$ is required to have the natural numbers as type. This defines the domain of the function. Then one may write the application $(\lambda x \cdot x+2) 3$, since 3 has the same type as $x$. But it would be quite unnatural to write the application $(\lambda x \cdot x+2)$ a, where a represents a vector in $R_{3}$.

In AUT-SL and in $A$, expressions have to obey the applicability condition, like in Automath. This condition is sufficiently strong to guarantee normalizability (even a weaker condition suffices, see Section I.6).

We note that Automath has the property that assignment of a type to an expression of degree 3 is different to that for expressions of degree 2. Expressions of degree 3 have lambda-structured types, whereas expressions of degree 2 all have the same type, viz. 
the expression denoted by the underlined symbol type. (This symbol type is the Automath version of the symbol $\tau$ used in our system $\Lambda$.) As an illustration we give an example in lambda-notation. Suppose that the term $\lambda x \cdot x+2$ has Nat as type for $x$, and type as type for Nat. Then $\lambda x \cdot x+2$ has degree 3 . In the manner of Automath it has $\lambda x \cdot$ Nat as type. The latter expression, having degree 2 , has as type the expression type.

In AUT-SL and in $\Lambda$, however, the assignment of types to expressions of any degree $\geq 2$ is treated in a uniform manner, comparable to the assignment of types to expressions of degree 3 in Automath.

If the term in the above example $(\lambda x \cdot x+2)$ were treated in the $\Lambda$-way, its type would again be $\lambda x \cdot N a t$, but the type of $\lambda x \cdot N a t$ would be $\lambda x \cdot \tau$.

We note that an extension of Automath, called AUT-QE ("Automath with quasi-expressions", see [6]), has more expressions of degree 1 than only type; it admits as expressions of degree 1 some of those admitted in AUT-SL and $\Lambda$. However, AUT-QE allows a choice to be made for some expressions of degree 2, between essentially different types.

Again using the above example as an illustration: in the manner of AUT-QE one may choose either $\lambda x$.type or type as type of $\lambda x \cdot N a t$.

It is to be noted that the above-mentioned difference between Automath (or AUT-QE) and AUT-SL (or A) has the important consequence that neither Automath nor AUT-QE is a subsystem of AUT-SL (or $\Lambda$ ). The results for $\Lambda$ obtained in this thesis are therefore not immediately transferable either to Automath or to AUT-QE.

Normalization for a simpler form of $A U T-Q E$, which does form a subsystem of AUT-SL, was proved by Van Benthem Jutting [22], using the norm introduced in this thesis (we shall call this norm $\rho$; cf. Section 1.6$)$. The normalization theorem of this thesis is a generalization of that of [22]. 
Strong normalization for a system resembling Automath was recently studied by R.C. de Vrijer on the basis of Tait's ideas exposed in [21], and for Automath and AUT-QE by D.T. van Daalen (private communications).

The uniqueness of normal form has only been proved with respect to $B$-reduction. Uniqueness of normal form with respect to $B-n-r e d u c t i o n$ is as yet an open question (see also section 1.6).

\section{CHANGE OF NOTATIONAL CONVENTIONS}

In the lambda-notation as usually employed the quantifiers (such as $\lambda x$ ) are written to the left of the expressions they operate upon, whereas applications are written to the right. This corresponds to mathematical notational traditions to write quantifiers (such as $\forall_{x}, \Sigma_{n=1}^{\infty}, \ldots$ ) to the left, and to write the argument of a function $f$ to the right (as in $f(a)$ ).

In ordinary mathematics these two kinds of operations have nothing in common, but in lambda-calculus they are closely related

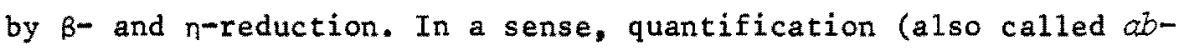
straction) and application are inverse operations. Sequences of such operations can be applied in various orders, and it is most convenient to write them all on the same side of an expression, thus showing clearly in which order the expression has been formed from its constituents.

In Automath applications and abstractions are all written to the left. Instead of writing abstractions in the form $\lambda x$, Automath writes $[x, A]$, in which A stands for the type of the variable $x$. Applications are indicated by witing the expression in braces; instead of the usual mathematical notation $f(a)$ we write $\{a\} f$.

For example: the term given in lambda-notation as $(\lambda x \cdot x+2) 3$ reads in Automath as: $\{3\}[x, N a t] p l u s(x, 2)$. (Here we assume that $x$ has as type the natural numbers, abbreviated Nat; a minor difference is that Automath uses only prefix notation for operators.) 
Note that the pair ][ indicates the possibility cf B-reduction. Sometimes, but not always, the pair $]\{$ indicates the possibility of n-reduction.

This notation for abstraction and application renders the use of parentheses ( ) entirely superfluous, since there can be no doubt as to the order in which abstractions and applications appear. The separation dot as used in $\lambda x \cdot x+2$ disappears as well. Automath uses the parentheses ( ), but for a different purpose.

In AUT-QE, AUT-SL and in the system $\Lambda$ which we shall develop in this thesis, these slightly different notational conventions are also adopted.

§ 6. SUMMARY OF THE CONTENTS OF THIS THESIS

This thesis contains a chapter on the formal system $\Delta$ (Chapter II) and a chapter on the formal system $\Lambda$ (Chapter III). In the latter chapter we develop the main results of the thesis.

System $\Lambda$ forms part of system $\Delta$, containing those expressions of $\Delta$ which obey the applicability condition (explained in Section I.4).

We shall now discuss the contents of Chapter II. There we define expressions inductively by: $x$ and $\tau$ are expressions; $[x, A] B$ and $\{A\} B$ are expressions if $A$ and $B$ are so ( $x$ is a variable).

In system $\Delta$ we only include those expressions which are "distinctly bound", i.e. (1) which do not contain free variables and (2) which have distinct binding variables.

Our preference for bound (also called closed) expressions (expressions without free variables) is noticeable throughout this thesis. We give the following justification for this preference. We believe that in a typed lambda-calculus the feature of typing can only be meaningful if every typable expression has an effectively computable type. Since free variables have no traceable type in our system, this implies that only bound expressions are admissible. If in this thesis we deviate from this agreement by considering ex- 
pressions with free variables, this will be in cases in which it is clear from the context which types belong to these free variables.

The consequence of the above agreement is that many expressions under discussion begin with an abstractor chain Q. (An abstractor chain is a string of abstractors; an abstractor has the form $[x, A], A$ being an expression.)

The fact that we require all binding variables in an expression in $\Delta$ to be distinct has only practical reasons (cf. Section II. 5).

We stress that system $\Delta$ is not a typed lambda-calculus in the usual sense, since the types have no influence whatsoever on the formation of expressions. The types, which themselves have a lambdastructure, will only be treated as formal expressions. It is not until Chapter III, dealing with the restricted system $\Lambda$, that the types will play the usual rôle in the formation of expressions. This is due to the applicability condition imposed upon expressions in $A$.

We shall formulate the relations $\alpha-, \beta-$ and $n$-reduction inside $\Delta$ and we shall prove a number of properties of these reductions in the system $\Delta$ (in sections II.4, II.5 and II.7, respectively).

In Section II. 6 we shall consider some reductions related to Breduction. Our proof of strong normalization in $\Lambda$ (Section III.3) is based on these reductions. The more important one of these reductions will be called $B_{1}$-reduction.

We shall explain its characteristic property by reducing the term which we previously used as an example: $(\lambda x+x+2) 3$, or, in Automath-notation: $\{3\}[\mathrm{x}, \operatorname{Nat}] \mathrm{plus}(\mathrm{x}, 2)$ (cf. the previous section).

As for $B$-reduction, we have the relation $\{3\}[x, \operatorname{Nat}] p l u s(x, 2) \geq_{\beta}$ $\geq_{\beta}$ plus $(3,2)$. But with $\beta_{1}$-reduction, which we denote by $\geq_{\beta_{1}}$, we have: $\{3\}[x$, Nat $] p l u s(x, 2) \geq_{B}\{3\}[x$, Nat $]$ plus $(3,2)$. Here the part $\{3\}[x, N a t]$ is left intact on the right-hand side. (Actually $B_{1}-r e-$ duction is more complicated; see Section II.6.)

The following feature of $B_{1}$-reduction is worth noting: Application of $B$-reduction sometimes enables one to eliminate a non- 
normalizable subterm (in this respect we recall the example $(\lambda x \cdot(\lambda y \cdot y))\left(\omega_{2} \omega_{2}\right) \geq_{\beta} \lambda y \cdot y$ of Section I.2), but with $\beta_{1}$-reduction this is impossible.

In Section II. 6 we prove the Church-Rosser property for $\beta_{1}$ reductions, using a proof technique of Tait and Martin-Löf. This property implies the uniqueness of normal form for $\beta_{1}$-reductions. From the Church-Rosser property for $\beta_{1}$-reductions follows the Church-Rosser property for B-reductions (although the latter could also be proved directly).

Unfortunately the Church-Rosser property for $\beta$-n-reductions does not hold in our system $\Delta$. The trouble here arises from the typed character of our lambda-calculus. We explain this in greater detail in Section II.7. (However, we conjecture the Church-Rosser property for $\beta$-n-reductions in $\Lambda$; see the end of the present section.)

In Section II.7 we also prove a theorem concerning the "postponement of $n$-reductions" in a sequence of $B$ - and $n$-reductions, by means of a method suggested by Barendregt.

At the end of Section II. 7 we define lambda-equivalence for $\Delta$ : $A$ and $B$ are lambda-equivalent if there is a $C$ such that $A$ and $B$ reduce to $C$. This lambda-equivalence is not necessarily transitive since the Church-Rosser property for $B$-n-reductions does not hold in $\Delta$.

In Section II. 8 we define a formal type-operator called Typ, which assigns a type to an expression not ending in $\tau$. The action of this type-operator is syntactically simple and is in agreement with what we mentioned about the assignment of types in Section I.3.

In Section II. 8 we also define the degree-function Deg, which is in agreement with our description of degree as given in Section I.3. In our system $\Delta$ we can apply the type-operator Typ a finite number of times.

For each expression $A$ in $\Delta$ there is an $n \geq 0$ such that Typ $A$ ends in $\tau$, which implies that $\operatorname{Typ}^{n} A$ has no type. (Here $\operatorname{Typ}^{n} A$ is obtained by $\mathbf{n}$ applications of the type-operator.) This $\mathbf{n}$ is the 
degree of $A$ minus one. We define Typ ${ }^{*}$ A to be Typ ${ }^{n}$ A for that particular $n$.

We begin Chapter III with the definition of the formal system $\Lambda$ (Section III.1). Among the theorems in Section III.1 there is one which states that the type of an expression in $\Lambda$ again belongs to $\Lambda$.

In Section III. 2 we prove the normalization theorem for $A$. We use a norm $\rho$, which is a partial function on $\Delta$. The norm $\rho(A)$ for a certain $A$ in $\Delta$ is itself an expression in $\Delta$. The norm of $A$ is defined if A obeys a weak form of the applicability condition, which amounts to the following: for each part of an expression $E$ which has the form of a function $F$ applied to an argument $A:(1)$ F has a domain $D$, and (2) the norms of $A$ and $D$ are defined and equal (apart from $a$-reduction).

Applied to expressions for which the norm is defined (socalled $\rho$-normable expressions), the norm $\rho$ has two powerful properties: (1) If $A$ reduces to $B$, the norms of $A$ and $B$ are (essentially) equal, and (2) the norm of an expression is (essentially) the same as the norm of its type.

The norm $\rho(A)$ of a $p$-normable expression $A$ can be obtained by (1) replacing non-binding variables by their types, repeating this process until no non-binding variable remains, and (2) cancelling adjacent pairs $\{C\}[x, D]$.

We show in Section III.2 that a11 expressions in $\Lambda$ are $\rho^{-}$ normable. We subsequently show that each $\rho$-normable expression has a normal form for $\beta$-reductions. It follows in particular that $\Lambda$ is normalizable for $\beta$-reductions. It now easily follows that $\Lambda$ is also normalizable for $\beta-n-r e d u c t i o n s$. Our proofs show that the normal form of $A$ in $\Lambda$ is effectively (viz. primitively recursively) computable.

In Section III. 3 we prove strong normalization for $\Lambda$. We use the $\beta_{1}$-reduction introduced in Section II.6. We show that expressions in $\Lambda$ are normalizable for $\beta_{\text {, }}$-reductions, using the same methods as in the corresponding proof for $\beta$-reductions in Section III. 2 . By using the Church-Rosser property for $\beta_{1}$-reductions as proved in 
Section II. 6 we obtain the uniqueness of normal form for $B_{1}$-reductions.

The special features of $B_{1}$-reduction enable us to conclude strong normalization in $\Lambda$ for $\beta_{1}$-reductions from the normalization and the uniqueness of normal form. Strong normalization in $A$ for $B-$ reductions is a consequence, as well as strong normalization for Bn-reductions.

The uniqueness of normal form in $\Lambda$ is proved for $\beta$-reductions

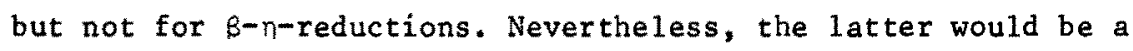
consequence if we could prove the following conjecture:

Conjecture I. In $\Lambda$ the Church-Rosser property holds for $B-n$-reductions.

(The difficulties in proving this arise in the same place where the corresponding statement for $\Delta$ turns out to be false; see Section IT.7.)

As to $\Lambda$, there is an important conjecture on closure:

Conjecture II. If $A$ is an expression in $\Lambda$ and if $A$ reduces to $B$, then $B$ is an expression in $\Lambda$.

In [15] we stated this as a theorem, but the proof turned out to be incorrect. The latter conjecture has no influence upon the results in this thesis; it is, however, of importance for the construction of an efficient checking-algorithm for expressions in $\Lambda$. 


\section{CHAPTER II, THE FORMAL SYSTEM $\triangle$}

\section{§ 1. ALPHABET AND SYNTACTICAL VARIABLES}

We use the following symbols as our alphabet:

(i) an infinite set of (individual) variables:

$\alpha, \beta, \gamma, \alpha_{1}, \beta_{1}, \gamma_{1}, \ldots$;

(ii) a single constant, called the base: $\tau$;

(iii) the improper symbols: $[],,\{\},, \ldots$

As syntactical variables denoting certain well-structured symbol strings (possibly empty) we use small Latin letters $a, b$, $c, \ldots$ and Latin capitals $A, B, C, \ldots$ (primed or subscripted if required). In special definitions, called Notation Rules, we restrict the use of some syntactical variables (and its primed or subscripted variants).

For example, we agree upon:

Notation Rule 1.1. As a syntactical variable for arbitrary strings of symbols from the alphabet we use the Latin capital S.

Such a string can be empty. The empty string itself is denoted by $\phi$.

Notation Rule 1.2. As syntactical variables for individual variables we use the small Latin letters $x, y$ and $z$. (Instead of "individual variable" we often say "variable".)

Hence from now on each use of a syntactical variable $S$ (or $S_{1}$, $S^{\prime}$, etc.) denotes a string of symbols from the alphabet, and each use of a syntactical variable $x$ (or $y, x_{1}$, etc.) denotes an individual variable.

It is usual to build strings of symbols from the alphabet and syntactical variables, concatenated. For example, $[x, \alpha][y, \alpha] x$ is such a string. We shall call this kind of string a mixed string. 
Equality of mixed strings will be expressed in the discussion language by the symbol $\equiv$. For example, if we wish to express that the strings $S$ and $[x, \alpha] \beta$ are the same, we write $S \equiv[x, \alpha] \beta$. The symbol $\neq$ is the negation of $\equiv$.

There are said to be two "occurrences" of $\alpha$ in the string $[\alpha, \beta] \alpha$. We shall formalize this notion occurrence. We define that $S^{\prime \prime}$ occurs in $S$ after $S^{\prime}$ if there is an $S^{\prime \prime}$ such that $S \equiv S^{\prime} S^{\prime \prime} S^{\prime \prime}$. Hence, in the above example: $\alpha$ occurs in $[\alpha, \beta] \alpha$ after $[$, and $\alpha$ occurs in $[\alpha, \beta] \alpha$ after $[\alpha, \beta]$. In this manner we can distinguish between occurrences.

$$
s \equiv s^{\prime} s_{1} s^{\prime \prime} \equiv s^{\prime} s^{\prime \prime} S_{2} s^{\prime \prime} s^{\prime \prime \prime} \text {. }
$$

The following statement is clear: If $S_{1}$ occurs in $S$ after $S^{\prime}$ and $s_{2}$ occurs in $s_{1}$ after $S^{\prime \prime}$, then $s_{2}$ occurs in $S$ after $S^{\prime} S^{\prime \prime}$.

Consider the mixed string $[x, y] x$, in which there are two occurrences of $\mathrm{x}$. If $\mathrm{x}$ denotes $\alpha$, then $[\mathrm{x}, \mathrm{y}] \mathrm{x}$ denotes $[\alpha, \mathrm{y}] \alpha$ : both occurrences of $\mathrm{x}$ are replaced by $\alpha$. If, moreover, $\mathrm{y}$ denotes $\beta$, then $[x, y] x$ denotes $[\alpha, \beta] \alpha$. It is, however, also possible that both $x$ and $y$ denote $\alpha$. Then $[x, y] x$ denotes $[\alpha, \alpha] \alpha$ (see also Schoenfield $[20, p .7])$.

Syntactical variables are used in two hardly distinguishable Rule' rôles: as abbreviations ("We abbreviate $[\mathrm{x}, \alpha] \beta$ as $\mathrm{S}^{\prime \prime}$ ) and as variables ("Let $S$ be a string of the form ..."). It is also good usage to state something in the nature of: "Let $A \equiv[x, B] C$ ", meaning: "Assume that $A$ has the form $[x, B] C$ for certain $x, B$ and $C$ " (in this manner one economizes in the use of the existential quantifier).

We shall define many specific sets and relations in an inductive manner (see Schoenfield [20, p. 4]). The proof technique linked with this kind of definition, which amounts to induction on the construction, is of ten called (somewhat confusingly) induction on the length of proof (or induction on theorems, see Schoenfield [20, p. 5]).

We shall call an application of one rule of the inductive definition a derivation-step. If a relation is defined inductively by a number of rules, then the relation is also said to be generated by these rules. When speaking of a transitive (or reflexive, etc.) relation generated by a number of rules, one wishes to express that 
the rule of transitivity (or reflexivity, etc.) is to be added to that number of rules.

If $S$ denotes a certain symbol string, then the length of $S$ is the number of symbols in that string. We denote the length of $S$ by $|s|$. For example, if $s \equiv[\alpha, \beta] \alpha$, then $|s|=6$.

\section{§. EXPRESSIONS}

The expressions of our systems are inductively defined as follows (we use the word expression rather than the words term or type):

Definition 2.1.

(1) A variable is an expression.

(2) $\tau$ is an expression.

(3) If $x$ is a variable and if $A$ and $B$ are expressions, then $[x, A] B$ is an expression.

(4) If $A$ and $B$ are expressions, then $\{A\} B$ is an expression.

Note that this definition gives a unique construction of an expression.

Notation Rule 2.2. As syntactical variables for expressions we use the Latin capitals A, B, C,...N,

Definition 2.3. A symbol string of the form $[x, c]$ is called an abstractor, a symbol string of the form $\{D\}$ an applicator. A tambda-phrase is either an abstractor or an applicator. A (possibly empty) string of abstractors (applicators, $\lambda$-phrases) is called an abstractor chain (an applicator chain, a lambda-phrase chain). $\square$

Notation Rule 2.4. As a syntactical variable for abstractor chains we use the Latin capital $Q$, for applicator chains the Latin cap$i$ tal $R$ and for $\lambda$-phrase chains the Latin capital $P$. 
The number of entries in a string forming an abstractor chain $Q$ (an applicator chain $P$, a lambda-phrase chain $R$ ) is denoted by $\|Q\|$ ( $\|P\|,\|R\|$ respectively). Hence $\|Q\|=0$ if $Q \equiv \phi$, and $\|\mathrm{Q}[\mathrm{x}, \mathrm{C}]\|=\|\mathrm{Q}\|+1$.

An expression $B$ can be a subexpression of an expression A, denoted $B$ C A. This relation is inductively defined as follows:

Definition 2.5.

(1) $A \subset A$.

(2) If $C \subset A$ or $C \subset B$, then $C \subset[X, A] B$ and $C \subset\{A\} B$.

Note: if $B \subset A$, then $A \equiv S_{1} B S_{2}$, i.e.: a subexpression of an expression $A$ is an expression which forms a connected part of $A$.

Instead of $B \subset A$ we sometimes say: $A$ contains $B$. If $B \subset A$ and $B \neq A$, we call $B$ a proper subexpression of $A$.

Theorem 2.6. If $F \subset E$ and $E \subset D$, then $F \subset D$.

Proof. Induction on $|D|$.

If $B \subset A$, then $B$ occurs in $A$, but there may evidently be more occurrences of $B$ in $A$. In the following we wish to be able to distinguish between such occurrences of $B$ in $A$. We shall indicate the occurrence meant by saying " $B \subset A$ after $S$ " if $B \subset A$ and $B$ occurs in $\mathrm{A}$ after $\mathrm{S}$.

Definition 2.7. Let $B$ occur in $A$ after $s$, and let $C$ occur in $A$ after $\mathrm{S}_{2}$. We call these occurrences disjoint if either $\mathrm{S}_{2} \equiv \mathrm{S}_{1} \mathrm{BS}^{\prime}$ or $s_{1} \equiv s_{2} C s^{\prime \prime}$.

Theorem 2.8. Let $B$ occur in $A$ after $S_{1}$, let $C$ occur in $A$ after $\mathrm{S}_{2}$, let $B \subset A$ and $C \subset A$. Then (1) $B$ and $C$ occur disjointly in $A$ or (2) $B \subset C$ or (3) $C \subset B$.

Proof. Induction on the length of proof of $B \subset A$.

Let $B \subset A$ after $S$. We shall inductively define the factor of $A$ with respect to $S$ and $B$ (denoted $A \mid\langle S ; B\rangle$ ) in definition 2.9. 
In this definition the occurrence of $B$ meant is precisely described.

However, it will of ten be clear from the context which occurrence of $B$ is meant in case $B \subset A$. In that case the precise indication of this occurrence is superfluous, and instead of $A \mid\langle S ; B\rangle$ we shall write $\mathrm{A} \mid \mathrm{B}$.

Informally we can concentrate the inductive definition of $A \mid B$, under the condition that in each of the following rules the occurrences of $B$ under discussion are "in corresponding places":

(1) If $A \equiv B$, then $A \mid B \equiv A$.

(2) If $A \equiv[X, C] D$, then $A|B \equiv C| B$ in case $B \subset C$ and $A \mid B \equiv[x, C](D \mid B)$ in case $B \subset D$.

(3) If $A \equiv\{C\} D$, then $A|B \equiv C| B$ in case $B \subset C$ and $A|B \equiv D| B$ in case $B \subset D$.

For a description of a characteristic property of $A \mid B$, which justifies its introduction, see the following section (after $\mathrm{Th}$. 3.6).

The formal inductive definition of $A \mid\langle S ; B\rangle$ is the following:

Definition 2.9. Let $B \in A$ after $S$.

(1) If $\mathrm{A} \equiv \mathrm{B}$, then $\mathrm{A} \mid\langle\mathrm{S}$; $\mathrm{B}\rangle \equiv \mathrm{A}$.

(2) Let $A \equiv[x, C] D$. If $B \subset C$ after $S_{1}$ and $\left[x, S_{1} \equiv S\right.$, then

$A|\langle S ; B\rangle \equiv C|\left\langle S_{1} ; B\right\rangle$. If $B \subset D$ after $S_{2}$ and $[x, C] S_{2} \equiv S$, then $A \mid\langle S ; B\rangle \equiv[X, C]\left(D \mid<S_{2} ; B>\right)$.

(3) Let $A \equiv\{C\} D$. If $B \subset C$ after $S_{1}$ and $\left\{S_{1} \equiv S\right.$, then $A|\langle S ; B\rangle \equiv C|\left\langle S_{1} ; B>\right.$. If $B \subset D$ after $S_{2}$ and $\{C\} S_{2} \equiv S$, then $A|\langle S ; B\rangle \equiv D|<S_{2} ; B>$.

Note: the parentheses ( ) in $[x, C]\left(D \mid<S_{2} ; B>\right.$ ) belong to the discussion language and are meant to fix the scope of $\mid$.

Let $B \subset A$ after $S$. It will be clear that $B \subset A \mid B$, or, a fortiori: $A \mid B$ ends in $B$ (here, of course, $A \mid B$ is meant to be $A \mid\langle S ; B\rangle)$. It is also evident that $A \mid B \equiv Q B$ and $(Q A) \mid B \equiv Q(A \mid B)$.

We state the following theorens: 
Theorem 2.10. If $C \subset B \subset A$, then $(A \mid B)|C \equiv A| C$.

Proof. Induction on $|A|$.

Theorem 2.11. If $B \subset A$ and $A \mid B \equiv Q_{1}[x, C] Q_{2} B$, then $C \subset A$.

Proof. Induction on $|A|$, using Th. 2.6.

Theorem 2.12. If $E \equiv Q_{1}[x, C] D$ and $B \subset C$ after $S$, then $E \mid B \equiv Q_{1}(C \mid B)$ (here $E \mid B$ is $E \mid<Q_{1}[X, S ; B\rangle$ and $C \mid B$ is $C \mid\langle S ; B\rangle$ ).

Proof. Induction on $\left\|Q_{1}\right\|$.

Theorem 2.13.

(1) If $[x, C] D \subset A$ after $S_{1}$ and $B \subset D$ after $S_{2}$, then $A \mid B \equiv Q_{1}[x, C] Q_{2} B$ (here $A \mid B$ is $A \mid<S_{1}[x, C] S_{2} ; B>$ ).

(2) If $B \subset A$ after $S$ and $A \mid B \equiv Q_{1}[x, C] Q_{2} B$, then there is a $D$ such that $[x, C] D \subset A$ after $S_{1}, B \subset D$ after $s_{2}$ and $S \equiv s_{1}[x, C] s_{2}$ (here $A \mid B$ is $A \mid<S ; B>$ ).

Proof. In both parts of the theorem: induction on $|A|$.

We conclude with an inductive definition of the function Tail, which maps expressions to expressions:

Definition 2.14.

(1) Tail $(x) \equiv x$.

(2) $\operatorname{Tail}(\tau) \equiv \tau$.

(3) $\operatorname{Tai}([\mathrm{X}, A] B) \equiv \operatorname{Tail}(B)$.

(4) $\operatorname{Tail}(\{A\} B) \equiv \operatorname{Tail}(B)$.

Note that Tail(A) can only be a variable or $\tau$. An expression $A$ can always be written (uniquely) as $A \equiv P$ Tail (A) (in which $P$ denotes a $\lambda$-phrase chain, see Notation Rule 2.4). 


\section{3. BOUND EXPRESSIONS}

An occurrence of a variable in an expression can be a free, a bound or a binding occurrence. We shall introduce these well-known notions in our system too. An occurrence of a variable in an expression is binding if and only if that occurrence immediately follows an opening bracket $[$.

If $D$ contains an occurrence of $x$ (i.e.: $x \in D$ ), then that occurrence of $\mathrm{x}$ is either bound (and there is a unique binding occurrence of $x$ which binds that bound occurrence) or free. A formal description is given in the following inductive definition. In this definition we often encounter "corresponding" occurrences of $x$. For easy understanding we shall not use our formalism concerning occurrences (see Section II,1), but we shall introduce "a certain $x "$ and refer to it as "that $x "$.

\section{Definition 3.1.}

(1) $x$ is free in $x$.

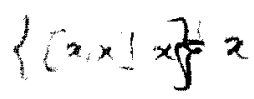

(2) Let a certain $x$ be free in $A$ or $B$. Then that $x$ is free in $\{A\} B$.

(3) Let a certain $x$ be free in $A$. Then that $x$ is free in $[y, A] B$ (both if $\mathrm{y} \equiv \mathrm{x}$ and if $\mathrm{y} \neq \mathrm{x}$ ).

(4) Let a certain $x$ be free in $B$. Then that $x$ is free in $[y, A] B$ if $y \neq x$, but that $x$ is bound in $[x, A] B$ (by the binding $x$ occurring in $[x, A] B$ after [] .

(5) Let a certain $x$ in $A$ be bound by a binding $x$ in $A$, or let a certain $x$ in $B$ be bound by a binding $x$ in $B$. Then that $x$ is bound by the corresponding binding $x$ in both $\{A\} B$ and $[y, A] B$ (also if $y \equiv x$ ).

The binding $x$ occurring in $[x, A] B$ after $[$ binds precisely the free $x^{\prime} s$ in $B$ (if any).

We shall mainly be interested in expressions in which no variable is free, called bound expressions (in the literature also called closed expressions). In bound expressions the same binding variable can occur in different instances. This cannot, however, 
give rise to confusion as to the connection between a bound variable and the binding variable by which it is bound.

Yet, for practical reasons, we wish to avoid such expressions. We call a bound expression in which all binding variables are different, adistinctly bound expression, and we restrict ourselves to the set of all distinctly bound expressions, which we call $\Delta$.

This is no essential restriction. Every interesting theory concerning bound expressions can be restricted to distinctly bound expressions.

Let $x \in D$ after $S$ and let this $x$ be bound in $D$. It follows from Def. 3.1 that we have $D \equiv s_{1}[x, E] F S_{2}$ such that $[x, E] F \subset D$, $x \subset F$ after $s_{3}, s_{1}[x, E] s_{3} \equiv S$ and the $x$ occurring in $D$ after $s_{1}[$ binds the $x$ occurring in $D$ after $S$. We shall call $[\mathrm{x}, \mathrm{E}]$ the binding apstractor of the bound $x$.

From this and Th. 2.13 (1) it follows:

Theorem 3.2. If $x \in D \in \Delta$, then $D \mid x \equiv Q_{1}[x, E] Q_{2} x$ and $[x, E]$ is the binding abstractor of the bound $x$ in $D$.

$[\because A] B$ din

It follows from Th. $2.13(2)$ :

Theorem 3.3. If for each $x \in D$ there are $Q_{1}, A$ and $Q_{2}$ such that $D \mid x \equiv Q_{1}[x, A] Q_{2} x$, then $D$ is a bound expression.

The following theorem expresses in an intricate manner the obvious observation that, in case $x \subset K \subset D \in \Delta$, the $x$ is bound by a binding abstractor either outside or inside $K$.

Theorem 3.4. If $x \in K \in D \in \Delta$, then either

(i) $D\left|K \equiv Q_{1}[x, A] Q_{2} K, D\right| x \equiv Q_{1}[x, A] Q_{2} Q^{\prime} x$ and $K \mid x \equiv Q^{\prime} x$, or

(ii) $\mathrm{K} \mid \mathrm{x} \equiv \mathrm{Q}_{1}[\mathrm{x}, \mathrm{A}] \mathrm{Q}_{2} \mathrm{x}$ and $\mathrm{D} \mid \mathrm{x} \equiv \mathrm{QQ}_{1}[\mathrm{x}, \mathrm{A}] \mathrm{Q}_{2} \mathrm{x}$.

In both cases $[x, A]$ is the binding abstractor of $x$ in $D$,

Proof. Let $\mathrm{D} \mid \mathrm{K} \equiv \mathrm{QK}$, then $\mathrm{D} \mid \mathrm{x} \equiv$ (by Th. 2.10) (D $\mid \mathrm{K})|\mathrm{x} \equiv(\mathrm{QK})| \mathrm{x} \equiv$ $Q(K \mid x) \equiv Q^{\prime} x$. Hence, by Th. 3.2, either $Q \equiv Q_{1}[x, A] Q_{2}$, or $Q^{\prime} \equiv Q_{1}[x, A] Q_{2}$. 
Theorem 3.5. If $Q\{A] B \in \Delta$, then $Q A$ and $Q B \in \Delta$; if $Q[x, A] B \in \Delta$, then QA $\in \Delta$.

Proof. Apply Th. 3.3.

Theorem 3.6. If $A \in \Delta$ and $B \subset A$ after $S$, then $A \mid B \in \Delta$. Proof. First assume that $x \in B$. Then by Th. 2.10: $(A \mid B)|x \equiv A| x=Q_{3}[x, C] Q_{4} x$. Next assume that $A \mid B \equiv Q B$ and $x \subset Q$. Then $Q \equiv Q_{1}[y, D] Q_{2}$ and $x \subset D$. By Th. 2.11: $D \subset A$. So $(Q B)\left|x \equiv Q_{1}(D \mid x) \equiv\left(Q_{1} D\right)\right| x \equiv(A \mid D)|x \equiv A| x \equiv$ $Q_{3}[x, C] Q_{4} x$. Apply Th. 3.3, and note that the binding variables in A|B must be distinct.

The above theorem states an essential feature of the factor $A \mid B$. If $A \in \Delta$ and $B \subset A$, then not necessarily $B \in \Delta$. But $A \mid B$, which is $Q B$ for a certain abstractor chain $Q$, "closes" $B$ in $\triangle$ by placing in front of $B$ those abstractors which necessarily bind all free variables of $B$. This, together with our previously uttered wish to restrict our expressions as much as possible to $\Delta$, justifies our introduction in Section II.2 of the factor A|B.

We continue with three theorems, related to one another.

Theorem 3.7. If $\mathrm{Q}[\mathrm{x}, \mathrm{A}] \mathrm{B} \in \Delta$ and $\mathrm{x} \notin \mathrm{B}$, then $\mathrm{QB} \in \Delta$.

Proof. Observe the various places where a variable $y(\neq x)$ can occur in $\mathrm{QB}$.

Theorem 3.8. If $\mathrm{QB} \in \Delta$ and $\mathrm{Q}[\mathrm{x}, \mathrm{A}] \mathrm{B} \in \Delta$, then $\mathrm{x} \notin \mathrm{B}$.

Proof. The assumption $x \in B$ leads to a contradiction.

Theorem 3.9. If $\mathrm{QA}$ and $\mathrm{QB} \in \Delta$, if the binding variables in $A$ and $B$ are distinct and if $x$ does not occur as a binding variable in $Q A$ or $Q B$, then $Q[x, A] B \in \Delta$.

Proof. Again observe the various places where a variable $y(\neq x)$ can occur in $Q[x, A] B$.

$$
\begin{aligned}
& D \equiv[y, A][x, 0] x \quad D \mid x=D ? \quad D \in \Delta \text { ? } \\
& \text { mit } \in \Delta \text { fing } 3.10
\end{aligned}
$$


In general: If $Q B$ and $Q P B \in \triangle$ and $x$ occurs as a binding variable in $P$, then not $x \subset B$. We say: $P$ has no binding influence on $B$. The description of $\Delta$, which we gave so far, began with (genera1) expressions and selected the distinctly bound expressions among these. This method is not so practical for theoretical investigations. In the following two theorems we shall indicate how we can compose expressions in $\Delta$ from expressions in $\Delta$. Or, rather, we shall show how expressions in $\Delta$ can be decomposed into smaller expressions, which also belong to $\Delta$.

Theorem 3.10 .

(1) $\tau \in \Delta$.

(2) If $Q A \in \Delta$ and if $x$ does not occur in $Q A$, then $Q[x, A] x \in \Delta$ and $\mathrm{Q}[\mathrm{x}, \mathrm{A}] \tau \in \Delta$.

(3) If $Q A$ and $Q y \in \Delta$, if $x$ does not occur in $Q A$ and if $x \neq y$, then $Q[x, A] y \in \Delta$.

(4) If $Q A$ and $Q B \in \triangle$ and if the binding variables in $A$ and $B$ are distinct, then $Q\{A\} B \in \Delta$.

Proof. It is trivial that $Q[x, A] x, Q[x, A] \tau, Q[x, A] y$ and $Q\{A\} B$ respectively are again expressions. These are also clearly bound expressions, moreover distinctly bound by the conditions given in the theoren.

We may consider the four parts of the previous theorem as derivation rules.

Definition 3.11. We call $\mathrm{k} \Delta$-constmactible if we can establish that $\mathrm{K} \in \Delta$ by a (finite) number of applications of the rules in Th. 3.10 .

The proof of the following theorem is technical. Yet it is interesting to see how we can establish $\Delta$-constructibility. For better understanding, we shall express the main lines at the end of the present section. 
Theorem 3.12. If $K \in \Delta$, then $K$ is $\Delta$-constructible.

Proof. Induction on $|K|$. If $|K|=1$, then $K \equiv \tau$ and $K$ is $\Delta$-constructible by rule (1).

Assume that $|\mathrm{K}|>1$, and let all distinctly bound expressions $K^{\prime}$ with $\left|K^{\prime}\right|<|K|$ be $\Delta$-constructible (first induction hypothesis). Then $K \equiv P_{1} P_{2} \ldots P_{n} T a i l(K)$ for some $n \geq 1$, where each of the $P_{i}$ is a lambda-phrase (i.e. either an abstractor or an applicator).

We can now prove the lemma: "For al1 $i$ for which $1 \leq i \leq n+1$ it holds that: $K \mid\left(P_{i} \ldots P_{n} T a i l(K)\right)$ is $\Delta$-constructible", by induction on $n+1-i$.

(1) Let $i=n+1$ (i.e. $\left.P_{i} \ldots P_{n} \equiv \phi\right)$. If $|K|$ Tail $(K)|<| k \mid$, then the first induction hypothesis leaves nothing to prove. If $|K| \operatorname{Tail}(\mathrm{K}) \mid=$ $|K|$, then $K \mid \operatorname{Tail}(K) \equiv K \equiv\left[z_{1}, E_{1}\right] \ldots\left[z_{n}, E_{n}\right] \operatorname{Tail}(K)$ for $n \geq 1$. (1a) Assume that $\operatorname{Tail}(K) \equiv x$. Then for exactly one $s: z_{s} \equiv x$. Abbreviate $\left[z_{1}, E_{1}\right] \ldots\left[z_{t}, E_{t}\right]$ as $Q_{t}$ for $0 \leq t \leq n$. We distinguish the cases $z_{n} \equiv x$ and $z_{\mathfrak{n}} \neq x$. If $z_{n} \equiv x$, then $k$ is $\Delta$-constructible by rule (2) since $Q_{n-1} E_{n} \in \Delta$ by Th. 3.5. If $z_{n} \neq x$, then $k$ is $\Delta-$ constructible by rule (3), since $Q_{n-1} E_{n} \in \Delta$ and $Q_{n-1} z_{n} \in \Delta$ (the latter by Th. 3.7).

(Ib) Assume that Tail(K) $\equiv \tau$. Then $Q_{n-1} E_{n} \in \Delta$ by Th. 3.5 and $\Delta-$ constructible by induction. By rule (2) we then find that $K$ is $\Delta-$ constructible.

(2) Let $1 \leq i \leq n$ and assume that $k \mid\left(P_{j} \ldots P_{n}\right.$ Tail(K)) is $\Delta$-constructible if $i<j \leq n+1$ (second induction hypothesis). If $|K|\left(P_{i} \ldots P_{n}\right.$ Tail $\left.(K)\right)|<| K \mid$, then again the first induction hypothesis leaves nothing to prove. So let $|K|\left(P_{i} \ldots P_{n} \operatorname{Ta} i l(K)\right) \mid=$ $|K|$. Then $k \equiv K \mid\left(P_{i} \ldots P_{n} \operatorname{Tail}(K)\right) \equiv\left[z_{1}, E_{1}\right] \ldots\left[z_{t}, E_{t}\right] P_{i} \ldots P_{n}$ $\operatorname{Tai1}(\mathrm{K})$.

(2a) Let $P_{i} \equiv\left[z_{t+l}, E_{t+l}\right]$. Then $K /\left(P_{i} \ldots P_{n} \operatorname{Tail}(K)\right) \equiv$ $\mathrm{K} \mid\left(\mathrm{P}_{\mathrm{i}+1} \ldots \mathrm{P}_{\mathfrak{n}} \mathrm{Tail}(\mathrm{K})\right)$, which is $\Delta$-constructible by the second induction hypothesis.

(2b) Let $P_{i} \equiv\{F\}$. Then $Q_{t} F \in \Delta$ by Th. 3.5 and $\Delta$-constructible by the second induction hypothesis, and the same holds for $Q_{t} P_{i+1} \cdots P_{n} \operatorname{Tail}(\mathrm{K})$. Hence, by rule (4): 
$Q_{t}\{F\} P_{i+1} \ldots P_{n} \operatorname{Tail}(K) \equiv K$ is $\Delta$-constructible.

From this lemma it follows that $K\left|\left(P_{1} \ldots P_{n} T a i l(K)\right) \equiv K\right| K \equiv K$ is $\Delta$-constructible.

(In this proof we did not check the conditions concerning variables in rules (1) to (4). It is easy to see that these are fulfilled in the appropriate places.)

From Th. 3.10 and Th. 3.12 follows that rules (1) to (4) generate the relation $K \in \Delta$. Hence, we can consider these four rules as a second definition of $\Delta$. The advantage of the latter is that we have an inductive definition of $\Delta$, whereas the original definition was restrictive with respect to the set of all expressions.

In a proof by induction the four rules of Th. 3.10 are much easier to use. The notion "induction on the length of proof" usually refers to a proof (in fact a construction) produced by an inductive definition, as is the second definition of $\Delta$. From now on we shall refer to this latter definition when giving a proof by "induction on the length of proof of $\mathrm{K} \in \Delta$ ".

Note that, given a $\mathrm{K} \in \Delta$, only one of the derivation steps in Th. 3.10 can give $K \in \Delta$ as a conclusion (if $K \equiv \tau$, then this must be rule (1). If $K \equiv Q\{A\} B$, then this must be rule (4). If $K \equiv Q \tau$ and $Q \neq \phi$, then this must be part 2 of rule (2). If $K \equiv Q x$ and $Q \equiv Q^{\prime}[X, A]$, then this must be part 1 of rule (2). If $K \equiv Q x$ and $Q \equiv Q^{\prime}[y, A]$ for some $y \neq x$, then this must be rule (3)).

The proof of the previous theorem suggests how we can establish that $K \in \Delta$ by using the four rules of $T h .3 .10$ (i.e. that $K$ is $\Delta-$ constructible). We can express this in words as follows:

Let $K$ be a distinctly bound expression. (1) we first establish that $K / T a i l(K)$ is $\Delta$-constructible: (la) if $\operatorname{Tail}(\mathrm{K}) \equiv \mathrm{x}$, then find the binding abstractor $[\mathrm{x}, \mathrm{A}]$ in $\mathrm{K}$ $\chi$ which binds $\mathcal{X}$, establish that $\mathrm{K} \mid \mathrm{A} \equiv \mathrm{QA}$ is $\Delta$-constructible, and apply rule (2) to obtain $Q[x, A] x \in \Delta$. Let $Q_{1}^{\prime}, Q_{2}^{\prime}, \ldots, Q_{k}^{\prime}$ be the abstractors occurring in $k \mid T a i l(K)$ "between" $[\mathrm{X}, A]$ and $\mathrm{x}$. Insert these abstractors, starting with $Q_{1}^{\prime}$ (from left to right), by in-

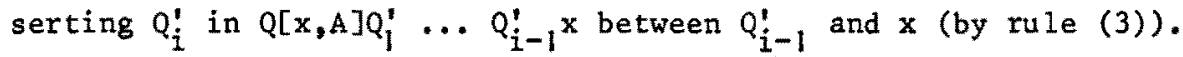


In this manner we establish that $K \mid$ Tail(K) is $\Delta$-constructiblc. (lb) If $\operatorname{Tail}(K) \equiv \tau$, then $\mathrm{K} \mid \operatorname{Ta} \mathrm{K}(\mathrm{K}) \equiv \tau$ and we may use rule (I) immediately, or $K \mid \operatorname{Tai} 1(K) \equiv Q[x, A] \tau$.

In the latter case: establish that $Q A$ is $\Delta$-constructible, and apply rule (2) to establish that $K \mid T a i l(K)$ is also $\Delta$-constructible. (2) We established that $K \mid \operatorname{Tail}(K) \equiv\left[x_{1}, A_{1}\right] \ldots\left[x_{n}, A_{n}\right]$ Tail $(K)$ is $\Delta^{-}$ constructible. In $K$ we find applicators $\left.\left\{B_{1}\right\}, \ldots, B_{\ell}\right\}$ "between" the abstractors $\left[x_{i}, A_{i}\right]$. Insert these $B_{i}$, starting with $B_{\ell}$ and ending with $B_{1}$ (from right to left) in the appropriate places, using rule (4). In this manner we establish that $\mathrm{K}$ is $\Delta$-constructible.

(Note the following. If we establish that $K$ is $\Delta$-constructible, then we use the $\Delta$-constructibility of $K \mid E$ for all $E \in K$. We can prove this by induction on the length of proof of $K \in \Delta_{\text {.) }}$ )

\$ 4. REPLACEMENT, RENOVATION AND $\alpha$-REDUCTION

If we replace a certain variable $\mathrm{x}$ in all its occurrences (free, bound or binding) in an expression $A$ by a variable $y$, then we denote the result of this replacement by $((x:=y)) A$. An inductive definition of simple replacement is the following (induction here is on the length of the expression):

Definition 4.1. For each pair $x$ and $y,((x:=y))$ is a function from expressions to expressions.

(1) $((x:=y)) x \equiv y ;((x:=y)) z \equiv z$ if $z \neq x ;((x:=y)) \tau \equiv \tau$.

(2) $((x:=y))[z, A] B \equiv[((x:=y)) z,((x:=y)) A]((x:=y)) B$.

(3) $((x:=y))\{A\} B \equiv\{((x:=y)) A\}((x ;=y)) B$.

The simple replacement of certain variables by others will be used for making the binding variables in an expression distinct. This we shall call the renovation of the expression.

Renovation is in fact nothing but a repeated renaming of variables. Renaming does not affect relevant properties of expressions 
(under a reasonable interpretation of "variable"; see also what we said concerning $\alpha$-equivalent terms in Section I.l).

We have maintained names for variables for reasons of tradition and legibility. This is at the expense of the renovation selector (to be introduced in this section) and the so-called $\alpha$ reduction (our wishes concerning bound expressions in the previous section had nothing to do with names for variables, but with our dislike of the occurrence of free variables; the additional wish to have distinctiy bound expressions, however, does concern names for variables, and leads us to introduce renovation).

We shall discuss the process of renovation. The mathematical meaning of renovation is not profound. If one dislikes a formalization of an intuitively clear concept, one can continue reading at Def. 4.4 (concerning $\alpha$-reduction).

There is, of course, one precaution which one must take in the renovation process: the relation between bound and binding variables should remain unaffected in a natural way. For example, in $[x, y][x, x] x$ the binding $x$ occurring after $[$ binds the bound $x$ occurring after $[x, y][x$, ; the binding $x$ occurring after $[x, y][$ binds the bound $x$ occurring at the end of the expression. In changing this expression into an expression with distinct binding variables, we might obtain: $[x, y][z, x] z$ for a certain $z \neq x$.

Such a variable $z$ as introduced in the latter example by the renovation process plays a special rôle. It has to be chosen with care. At any rate it should be different to all binding variables in the expression under discussion, or, as we shall say: it has to be fresh with respect to that expression.

We introduce the renovation selector $\mathrm{Fr}_{\mathrm{V}}$, operating on expressions. In using the renovation selector $\mathbf{F r}_{V}$ with an expression A we have it preceded by a lambda phrase chain $P$, giving $\mathrm{PFr}_{\mathrm{V}} \mathrm{A}$. The subscript $V$ denotes a finite set of variables, which can be empty. We shall not specify the variables belonging to $V$ until the following section, where we use $\mathrm{Fr}_{\mathrm{V}}$ in the formal definition of substitution. 
The expression PFr $\mathrm{V}^{\mathrm{A}}$ can informally be described as being PA', where (1) $A^{\prime}$ is obtained from $A$ by renovation of $A$ and (2) the fresh variables chosen during this renovation do not occur in $P$ or $\mathrm{V}$ and are mutually distinct.

The following inductive definition gives a formalization of this concept:

Definition 4.2. Let $\mathrm{V}$ be a finite set of variables.

(1) $\operatorname{PFr}_{V^{X}} \equiv P x ; \operatorname{PFr}_{V^{T}} \equiv \operatorname{Pr}$.

(2) $\operatorname{PFr}_{V}([y, B] C) \equiv P\left[z, B^{\prime}\right] F_{V}(((y:=z)) C)$, with $P B^{\prime} \equiv \operatorname{PFr}_{V} B$, while $z$ does not occur in $P B^{\prime}$ and $z \notin V$.

(3) $\operatorname{PFr}_{V}(\{B] C) \equiv P\left\{B^{\prime}\right\} F r_{V} C$, with $P B^{\prime} \equiv \operatorname{PFr}_{V}{ }^{B}$.

From the above definition we see that the renovation of an expression takes place from left to right. For instance, the renovation resulting in $\operatorname{PFr}_{V}(\{B\} C)$ first requires the renovation resulting in $\mathrm{PFr}_{V} \mathrm{~B}$, and subsequently the one resulting in $\left.\mathrm{P}^{\prime} \mathrm{B}^{\prime}\right\} \mathrm{Fr}_{V} \mathrm{C}$. This implies that the fresh variables chosen in the renovation process are mutually distinct.

of course uncertainties remain as to the choice of fresh variables. An order in the set of all variables could turn the selector $\mathrm{Fr}_{\mathrm{V}}$ into an operator. We shall, however, not push the formalization this far.

In the following section we shall describe substitution by the aid of the renovation selector, and we shall in turn use substitution in describing the $\beta$-reduction. Our use of the renovation selector is meant to keep an expression distinctly bound after Breduction. We shall use the renovation selector in typing an expression, as is described in Section II.8, with the same purpose.

We usually begin renovation with $V=\phi$ (here, of course, $\phi$ denotes the empty set and not the empty string).

Definition 4.3. $\mathrm{PFrA} \equiv \operatorname{PFr}_{\phi} \mathrm{A}$. 
In fact PFrA is the concatenation of $P$ and $F_{W} A$, where $W$ is the set of variables occurring in $P$.

Let $\operatorname{PFr}_{V} A \equiv \mathrm{PA}^{\prime}$ be the result of a renovation. If we again write $\operatorname{Fr}_{V} A$, in the same context, we do not require a new renovation, but mean $A^{\prime}$. If we wish another renovation in the same context, then we supply $\operatorname{Fr}_{V}$ with primes: $\operatorname{PFr}_{V}^{A}$ can be such a new renovation. We shall now define the a-reduction relation. For an informal discussion of $\alpha$-reduction see section $I .1$. We restrict a-reduction to expressions in $\Delta$ :

Definition 4.4. $\alpha$-reduction, denoted by $\geq_{\alpha}$, is the transitive relation generated by:

(1) If $A \in \Delta$ and if $y$ does not occur in $A$, then $A \geq((x:=y)) A . \square$

The $\alpha$-reduction is clearly an equivalence relation (reflexivity: take $x$ to be a variable which does not occur in $A$; symmetry: note that $x$ does no longer occur in $((x:=y)) A)$.

If two expressions are related by $\alpha$-reduction $\left(A \geq_{\alpha} B\right.$ ), we speak of "the a-reduction $A \geq_{\alpha} B$ ". This is clearly abuse of language, although it cannot give rise to confusion.

A renaming of a single variable in a distinctly bound expression is called a single-step a-reduction, denoted as $A \geq_{\alpha}^{\prime} B$ (so $A \geq_{\alpha}^{\prime} B$ if and only if $A \in \Delta$ and $B \equiv((x:=y)) A$ where $x$ occurs as a binding variable in $A$ and $y$ does not occur in $A$ ).

The following theorems are trivial:

Theorem 4.5. If $\mathrm{PA} \in \Delta$, then $\mathrm{PFr}_{V} \mathrm{~A} \in \Delta$; if $\mathrm{A} \in \Delta$ and $\mathrm{A} \geq_{\alpha} \mathrm{B}$, then $B \in \Delta$ and $|A|=|B|$.

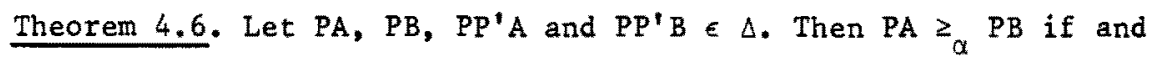

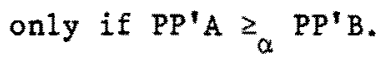




\section{SUBSTITUTION AND B-REDUCTION}

Substitution is an operation acting on expressions. We denote "the result of the substitution of $A$ for $x$ in $B$ " by $(x:=A) B$. One can use several definitions of substitution which are equivalent.

We shall use the definitions given in Def. 5.1 and Def. 5.2. These definitions of substitution can informally be described as follows: $P(x:=A) v^{B}$ is the expression which we obtain from $P B$ by replacing all free $x^{\prime} s$ in $B$ with renovations of $A$, in which the fresh variables are chosen in the following manner: they have to be mutually distinct for all renovations of A replacing the free $x^{\prime} s$, and they have to be distinct from the variables occurring in $B, P$ or $V$ (we do not replace the binding variables in $B$ by fresh ones). Here $V$ denotes a finite set of variables, which can be empty.

This careful dealing with fresh variables is necessary to guarantee that an expression with distinct binding variables has again distinct binding variables after $\beta$-reduction (to be defined in this section.); substitution is an essential part of $\beta$-reduction.

The following part of this section as far as Def. 5.5., will formalize the above notion of substitution. As with renovation, our formalization of substitution may be cumbersome to the reader. One may continue with Def. 5.5, without impairing understanding.

An inductive definition of $P(x:=A) V^{B}$ is the following (induction is here on the length of $B$ ):

Definition 5.1.

(1) $P(x:=A) v x \equiv P F r v^{A} ; P(x:=A) v^{y} \equiv P y$ if $y \neq x ; P(x:=A) v^{\tau} \equiv P r$.

(2) If $y \not x$, then $P(x:=A) v[y, B] C \equiv P\left[y, B^{\prime}\right](x:=A) v^{C}$, where $P B^{\prime} \equiv P(x:=A) W^{B}, W$ being the union of $V$ and the set of all variables occurring in $C$.

If $y \equiv x$, then $P(x:=A) V^{[y, B] C} \equiv P\left[y, B^{\prime}\right] C$, where $B^{\prime}$ is obtained as above.

(3) $P(x:=A) v^{\{B\} C \equiv P\left\{B^{\prime}\right\}(x:=A)} V^{C}$, where $B^{\prime}$ is obtained as above. $\square$ 
From the above definition we see that substitution (as renovation) takes place from left to right in an expression: for instance, the substitution resulting in $P(x:=A) V(B\} C$ first requires the substitution resulting in $P(x:=A) W^{B}$, and subsequently the substitution resulting in $P\left\{B^{\prime}\right\}(x:=A){ }_{V} C$.

In part (2) of the definition we see the importance of the subscript used in $(x:=A)$ : in executing the substitution resulting in $P(x:=A){ }_{W}{ }^{B}$ we must have at our disposal the set of variables occurring in $\mathrm{C}$ in order to be able to choose fresh variables different from the variables in $C$. The set $W$ contains the latter variables.

The set $V$ can be the empty set. When we begin a substitution, $V$ is usually empty.

Definition 5.2. $P(x:=A) B \equiv P(x:=A)_{\phi} B$.

In the above definitions there are two more or less unusal parts. Usually $(x:=A) x$ is defined as $A$; however, with a view to our wish to keep distinctly bound expressions distinctly bound after some substitution, we deviate from this. Next, one sometimes defines $(x:=A)[y, B] C$ as $[z,(x:=A) B](x:=A)(y:=z) C$, in either of the cases that $x \equiv y$ or $x \neq y, z$ being a fresh variable. The latter definition prevents so-called "confusion of variables" (cf. Curry and Feys [3, Ch. 3D2]; see also the example below), but gives rise to an additional amount of simple substitutions of variables of the form $(y:=z)$, which we find cumbersome.

In using Def. 5.1. and Def. 5.2. confusion of variables may occur if the use of the substitution operator is not restricted. For example: $[y, A](x:=y)[y, \tau] x \equiv[y, A][y, \tau] y$, where the final $y$ in the latter expression is influenced by $[y, \tau]$, and not by $[y, A]$ as it should be.

In general: confusion of variables may arise as a consequence of the substitution resulting in $P(x:=A) V B$ if a free variable $y$ of A (with $y \neq x$ ) occurs as a binding variable in $B$, and if there is a free $x$ in $B$ within the "scope" of that binding variable $y$. 
A sufficient condition for avoiding this is, that the free variables of $A$ do not occur as binding variables in $B$.

We use substitution only in the relation B-reduction, defined later in this section. The above condition is there fulfilled. Hence confusion of variables cannot arise in our system.

Note that $(x:=A)$ operates on free $x^{\prime} s,((x:=A))$ on all $x^{\prime} s$, and $\mathrm{Fr}$ on all binding variables in an expression.

We also define the substitution operator for lambda-phrase chains:

Definition 5.3. If $(x:=A) P \tau \equiv P^{\prime} \tau$, then $(x:=A) P \equiv P^{\prime}$.

One may interchange the substitution operator and the renovation selector under certain conditions:

Theorem 5.4. Let $\mathrm{PA} \in \Delta$ and $\mathrm{P}[\mathrm{x}, \mathrm{B}] \mathrm{D} \in \Delta$. Then $\operatorname{PFr}(x:=A) D \geq_{\alpha} P(x:=A) F r D$ if no binding variable of $D$ occurs in $A$. Proof. Induction on $|D|$, using Th, 4.5 and the lemma: " $((y:=z))(x:=A) C \equiv(x:=A)((y:=z)) C$ if $y \neq A$ (but for renaming)".

Substitution is used in the more interesting reduction in lambda-calculus called $\beta$-reduction, which we denote by $\geq_{\beta}$. The interpretation linked with $\beta$-reduction is the application of a function to an argument (see also the informal description in Section I.1).

We shall restrict $\beta$-reduction to expressions belonging to $\Delta$. This is unusal. One usually conceives of a reduction as a formal relation between expressions in which free variables may occur. It is only our preference for distinctly bound expressions which makes us choose the definitions given below.

Note that our $B$-reduction is not essentially different from the usual concept. The use of the $Q$ in Def. 5.5 is a little obscuring in this respect.

We first define single-step B-reduction, denoted by $z_{B}^{\prime}$ :

Definition 5.5. Single-step B-reduction is the relation generated by: 
(1) If $Q\{A\}[x, B] C \in \Delta$, then $Q\{A\}[x, B] C \geq_{\beta}^{\prime} Q(x:=A) C$.

(2) Let $Q\{A\} C$ and $Q\{A\} D \in \Delta$. If $Q C \geq_{B}^{\prime} Q D$, then $Q\{A\} C \geq_{B}^{\prime} Q\{A\} D$.

(3) Let $Q[x, A] C$ and $Q[x, B] C \in \Delta$. If $Q A \geq_{B}^{\prime} Q B$, then $Q[x, A] C \geq_{B}^{\prime} Q[x, B] C$.

(4) Let $Q\{A\} C$ and $Q\{B\} C \in \Delta$. If $Q A z_{B}^{\prime} Q B$, then $Q\{A\} C z_{B}^{\prime} Q\{B\} C$.

Note: one rule appears to be missing (viz.: let $Q[x, A] C$ and $Q[x, A] D \in \Delta$. If $Q C \geq_{B}^{\prime} Q D$, then $\left.Q[x, A] C \geq_{B}^{\prime} Q[x, A] D\right)$. But this is a derived rule, see Cor. 5.14 .

Rules (2), (3) and (4) in the above definition are called the monotony rules of single-step $\beta$-reduction; we call rule (1) the rule of elementary $\beta$-reduction.

If $K$ and $L$ are related by a single-step $B$-reduction, we obtain $L$ from $K$ by replacing a certain subexpression $\{A\}[X, B] C$ in $K$ by $(x:=A) C$. This is, in terms of interpretation, a single functional application.

If $K \geq_{B}^{\prime} L$, then we have a construction (a "proof") which establishes that relation according to Def. 5.5. Such a construction consists of one derivation step which is an elementary $\beta-$ reduction (rule (1) of Def. 5.5) followed by a number of derivation steps which are monotony steps (rules (2) to (4)). Note that a single-step $\beta$-reduction is achieved from a number of derivation steps.

We note that, since $Q\{A\}[x, B] C \in \Delta$, no free variable of $A$ can occur as binding variable in $C$. This is sufficient, as previously stated, to prevent "confusion of variables".

Definition 5.6. $\beta$-reduction is the reflexive and transitive closure of single-step $\beta-r e d u c t i o n$.

(This means that (1) if $K \geq_{\beta}^{\prime} L$, then $K \geq_{\beta} L$; (2) $K \geq_{\beta} K$;

(3) if $K \geq_{\beta} L$ and $L \geq_{\beta} M$, then $K \geq_{\beta} M_{\text {. }}$ )

If $A$ and $B$ are related by a single-step $\beta$-reduction, we speak of "the single-step $\beta$-reduction $A \geq_{B}^{\prime} B$ ". As with $\alpha$-reduction, this 
is abuse of language. Analogously we speak of "the B-reduction $A \geq{ }_{B} B^{\prime \prime}$.

If $A_{0} \geq A_{B}^{\prime}, A_{1} \geq_{B}^{\prime} A_{2}, \ldots, A_{n-1} \geq_{B}^{\prime} A_{n}$, we also write $A_{0} \geq_{B}^{\prime} A_{1} \geq_{B}^{\prime} \cdots \geq_{B}^{\prime} A_{n}$ or $A_{0} \geq_{B}^{n} A_{n}$. We call this a composite singlestep $B$-reduction, or an $n$-step $B$-reduction (a zero-step $B$-reduction has, of course, the form $\left.A \geq \geq_{B}^{0} A\right)$. If $A_{0} \geq_{B} A_{1}, \ldots, A_{n-1} \geq_{B} A_{n}$, we also write $A_{0} \geq_{B} A_{1} \geq_{B} \ldots \geq_{B} A_{n}$.

If follows from the definition of $\beta$-reduction that each $\beta-$ reduction $K \geq_{B} L$ can be presented as an $n$-step $\beta$-reduction $K \equiv A_{0} \geq_{B}^{\prime} \ldots \geq_{\beta}^{\prime} A_{n} \equiv$ L. This splitting is called a decomposition of a $\beta$-reduction.

Each of the monotony rules has the form: "If reduction (i) holds, then reduction (ii) holds". It is usual to call reduction (ii) the direct consequence of reduction (i). For example: $Q\{A\} C \geq_{B} Q\{A\} D$ is a direct consequence of $Q C \geq_{B} Q D$. We recall that "the length of proof of $\mathrm{K} \geq_{\beta}^{\prime} \mathrm{L}^{\prime \prime}$ is the total number of derivation steps in the proof of $K \geq_{B}^{\prime} L$.

A proof of $K \geq \geq_{B}^{\prime} L$ begins with an elementary $\beta$-reduction $Q\{A\}[x, B] C \geq_{B} Q(x:=A) C$. In this case we say that $Q\{A\}[x, B] C$ generates the single-step $\beta$-reduction $K \geq_{\beta}^{\prime}$ L. The following theorem holds:

Theorem 5.7. Let $\mathrm{K} \hat{\epsilon} \Delta$. Then $\mathrm{Q}\{\mathrm{A}\}[\mathrm{X}, \mathrm{B}] \mathrm{C}$ generates a single-step $B-$ reduction of the form $K \geq B$ if and only if $\{A\}[x, B] c \subset K$ and $Q\{A\}[X, B] C \equiv K \mid\{A\}[x, B] C$.

Proof. $\rightarrow$ : Induction on the length of proof of $K \geq_{\beta}^{\prime} L$. $\ll$ : We state the following zemma: "Let $K \equiv Q^{\prime} M \in \Delta$, and let $E \equiv\{A\}[X, B] C \subset M$. Then $K \mid E$ generates a single-step $B-r e d u c t i o n$ $K \equiv Q^{\prime} M \geq_{B}^{\prime} Q^{\prime} N "$. We prove this lema by induction on $|M|:$ (1) Let $M \equiv E$. Then $K \mid E \equiv K \equiv Q^{\prime}\{A\}[x, B] C \geq_{\beta}^{\prime} Q^{\prime}(x:=A) C$. (2a) Let $M \equiv[X, F] G$ and $E \subset F, C a l l K^{\prime} \equiv Q^{\prime} F \in \Delta$; then $E \subset F$, hence, by induction: $K^{\prime} \mid E \equiv Q^{\prime}(F \mid E)$ generates a single-step B-reduction $K^{\prime} \equiv Q^{\prime} F \geq_{\beta}^{\prime} Q^{\prime} F^{\prime}$. By applying monotony rule (3) it follows that $K^{\prime} \mid E$ also generates $Q^{\prime} M \equiv Q^{\prime}[x, F] G \geq_{B}^{\prime} Q^{\prime}\left[X, F^{\prime}\right] G$. Moreover, $R\left|E \equiv K^{\prime}\right| E$. 
(2b) Let $M \equiv[x, F] G$ and $E \subset G$. Take $K^{\prime} \equiv K \equiv Q^{\prime}[x, F] G \equiv Q^{\prime \prime} G$; then $E \subset G$, hence, by induction: $K^{\prime} \mid E$ generates a single-step B-reduction $Q^{\prime \prime} G \geq_{\beta}^{\prime} Q^{\prime \prime} G^{\prime}$, which can be rewritten as $Q^{\prime} M \geq_{B}^{\prime} Q^{\prime}[x, F] G^{\prime}$. (3a) Let $M \equiv\{F\} G$ and $E \subset F$. The proof is analogous to the one in case (2a), using monotony rule (4) instead of (3).

(3b) Let $M \equiv\{F\} G$ and $E \subset G$. Again the proof is analogous to the one in case (2a), now using monotony rule (2) instead of (3). This proves the lemma. The "if-part" of the theorem follows immediately from the lemma.

We state the "closure theorem for A with respect to singlestep B-reduction":

Theorem 5.8. If $K \in \Delta$ and $K \geq_{\beta}^{\prime} \mathrm{L}$, then $\mathrm{L} \in \Delta$. Proof. Induction on the length of proof of $K \geq_{B}^{\prime}$ L. Note that our definition of substitution for a variable with the aid of the renovation selector is essential. In the proof we can use Th. 3.2. $\square$

Corollary 5.9. If $K \in \Delta$ and $K z_{B} L$, then $L \in \Delta$.

One may conceive of the $\beta$-reduction relation, not as a relation between expressions, but as a relation between $\alpha$-equivalence classes (in the $\alpha$-equivalence class of $K$ we include all $K^{\prime}$ such that $\mathrm{K} \geq_{\alpha} \mathrm{K}^{\prime}$ ).

The following theorem gives a justification for this conception of $\beta$-reduction:

Theorem 5.10. Let $A \in \Delta, A \geq_{B}^{\prime} B$ and $A \geq_{\alpha} C$. Then there is a reduction $C \geq_{B}^{\prime} D$ such that $B \geq_{\alpha} D$.

Proof. It is sufficient to assume that $A \geq_{\alpha}^{\prime}$ C. Apply induction on the length of proof of $A \geq_{B}^{\prime} B$.

In the sequel we shall sometimes refer to the above conception of B-reduction, by inserting the words "but for a-reduction" in a statement (for example: "A $\geq_{\beta}$ B. but for $\alpha$-reduction" means that 
there are $A^{\prime}$ and $B^{\prime}$ such that $A \geq_{\alpha} A^{\prime} \geq_{\beta} B^{\prime} \geq_{\alpha} B$ ). However, we often omit the words "but for $\alpha$-reduction".

We shall proceed with a number of theorems, in which especially the rôle of the abstractor chain $Q$ in a $\beta$-reduction is considered, $Q$ occurring in the beginning of an expression. (The definition of \|P | was given after notation rule 2.4.)

Theorem 5.11. If $Q E \in \Delta, Q E \geq_{\beta}^{\prime} Q^{\prime} F$ and $\|Q\|=\left\|Q^{\prime}\right\|$, then $Q \equiv Q^{\prime}$ or $E \equiv F$. In the latter case $Q \equiv Q_{1}[x, K] Q_{2}, Q^{\prime} \equiv Q_{1}[x, L] Q_{2}$ and $Q_{1} K \geq_{B}^{\prime} Q_{1} L$.

Proof. Induction on the length of proof of $Q E \geq_{\beta}^{\prime} Q^{\prime} F$. There are four possible cases for the last derivation step in the proof of $Q E \geq_{\beta}^{\prime} Q^{\prime} F$. In three of these cases the conclusion is: $Q \equiv Q^{\prime}$. The fourth case is that the last derivation step in the proof of $Q E \geq_{\beta}^{\prime} Q^{\prime} F$ is: " $Q_{1} K \geq_{\beta}^{\prime} Q_{1} L$, so $Q E \equiv Q_{1}[x, K] M \geq_{B}^{\prime} Q_{1}[x, L] M \equiv Q^{\prime} F^{\prime}$. Now if $\|Q\| \leq\left\|Q_{1}\right\|$, then $Q \equiv Q^{\prime}$, and if $\|Q\|>\left\|Q_{1}\right\|$, then $E \equiv F$, $Q \equiv Q_{1}[x, K] Q_{2}$ and $Q^{\prime} \equiv Q_{1}[x, L] Q_{2}$.

Theorem 5.12. If $Q E \in \Delta$ and $Q E \geq_{Q}^{\prime} K$, then $K \equiv Q^{\prime} E^{\prime}$ for certain $Q^{\prime}$ and $E^{\prime}$ with $\left\|Q^{\prime}\right\|=\|Q\|$.

Proof. The reduction $Q E \geq_{\beta}^{\prime} \mathrm{K}$ must be the conclusion of one of the rules of Def. 5.5 It is easy to see that the statement of the theorem holds good in all these cases.

If a reduction $Q C \geq_{B} Q^{\prime} D$ is given, in which $\|Q\|=\left\|Q^{\prime}\right\|$, one can conceive of an accompanying "reduction" of $C$ to $D$. The following theorem shows this.

Theorem 5.13. If $Q C, Q_{0} C$ and $Q_{0} D \in \Delta, Q C \geq_{B} Q^{\prime} D$ and $\|Q\|=\left\|Q^{\prime}\right\|$, then $Q_{0} C \geq_{B} Q_{0} D$.

Proof. First assume that $Q C \geq_{\beta}^{\prime} Q^{\prime} D$. Then by Th. $5.11 Q \equiv Q^{\prime}$ or $C \equiv D$. In the latter case it is trivial that $Q_{0} C \geq_{B} Q_{0} D$. So assume $Q \equiv Q^{\prime}$. Then induction on the length of proof of $Q C \geq_{\beta}^{\prime} Q^{\prime} D$ and Th. 5.12 yield $Q_{0} C \geq_{B}^{\prime} Q_{0} D$. The general theorem follows. 
The apparently missing monotony rule, announced immediately af ter Def. 5.5, is a consequence:

Corollary 5.14. Let $Q C, Q D, Q[x, A] C$ and $Q[x, A] D \in \Delta$. If $Q C \geq_{B}^{t} Q D$, then $\mathrm{Q}[\mathrm{x}, \mathrm{A}] \mathrm{C} \geq \geq_{B}^{\prime} \mathrm{Q}[\mathrm{x}, \mathrm{A}] \mathrm{D}$.

We defined $\beta$-reduction as being transitive and reflexive. We shall now show that $\beta$-reduction is also monotonous:

Theorem 5.15. The monotony rules hold for B-reduction, i.e.:

(2) If $Q\{A\} C$ and $Q\left\{k_{3}\right\} D \in \Delta$, and $Q C \geq_{B} Q D$, then $Q\{A\} C \geq_{B} Q\{A\} D$.

(3) If $Q[x, A] C$ and $Q[x, B] C \in \Delta$, and $Q A \geq_{B} Q B$, then $Q[x, A] C \geq_{B} Q[x, B] C$.

(4) If $Q\{A\} C$ and $Q\{B\} C \in \Delta$, and $Q A \geq_{B} Q B$, then $Q\{A\} C \geq_{B} Q\{B\} C$.

Proof. We shall prove rule (2).

Since $Q C \geq_{B} Q D$, we know that $Q C \geq_{B}^{\prime} E_{1} \geq_{B}^{\prime} E_{2} \ldots \geq_{B}^{\prime} E_{n} \geq_{B}^{\prime} Q D$.

From Th. 5.12, Th. 5.13 and induction on $n$ it follows that there is also a reduction $Q C \geq_{\beta}^{\prime} Q F_{1} \geq_{\beta}^{\prime} Q F_{2} \ldots \geq_{\beta}^{\prime} Q F_{n} \geq_{B}^{\prime} Q D$. Repeated application of the corresponding monotony rule for single-step $\beta-$ reduction gives: $Q\{A\} C \geq_{B}^{\prime} Q\{A\} F_{1} \geq_{B}^{\prime} \ldots \geq_{B}^{\prime} Q\{A\} D$, hence Q\{A\}C $\geq_{B}$ Q\{A\}D.

The other two monotony rules for B-reduction can be proved analogously.

One may extend Cor. 5.14 to B-reduction:

Theorem 5.16. Let $Q C, Q D, Q[x, A] C$ and $Q[x, A] D \in \Delta$. If $Q C z_{B} Q D$, then $\mathrm{Q}[\mathrm{x}, \mathrm{A}] \mathrm{C} \geq_{B} \mathrm{Q}[\mathrm{x}, \mathrm{A}] \mathrm{D}$.

Theorem 5.17. If $Q C, P C$ and $P D \in \Delta, Q C \geq_{B} Q^{\prime} D$ and $\|Q\|=\left\|Q^{\prime}\right\|$, then $\mathrm{PC} \geq_{\beta}$ PD.

Proof. Let $\mathrm{PC} \mid \mathrm{C} \equiv Q_{0} C$, then $Q_{0} C \geq_{B} Q_{0} D$ follows from Th. 5.13. The theorem is proved by repeated application of monotony rule (2) for $\beta$-reduction (see Th. 5.15). 
Note: the converse of this theorem does not hold ("If PC, QC and $Q D \in \Delta$ and $P C \geq_{B} P D$, then $Q C \geq_{\beta} Q D$ ").

Example $(Q \equiv[x, \tau], P \equiv[x, \tau]\{x\})$ :

$[x, \tau]\{x\}[y, \tau]\{x][z, \tau] y z_{\beta}[x, \tau]\{x\}[z, \tau] x$, but not:

$[x, \tau][y, \tau]\{x\}[z, \tau] y \geq_{\beta}[x, \tau][z, \tau] x$.

Theorem 5.18. If $P\{A\}[x, B] C \in \Delta$, then $P\{A\}[x, B] C \geq_{\beta}^{\prime} P(x:=A) C$.

Proof. This is a consequence of the following:

$(P\{A\}[x, B] C) \mid\{A\}[x, B] C \equiv Q\{A\}[x, B] C \geq_{B}^{Q} Q(x:=A) C$. Apply Th.5.17.

Theorem 5.19. If $Q K, Q M$ and $Q^{\prime} M \in \Delta, Q K \geq_{B} Q^{\prime} L$ and $\|Q\|=\left\|Q^{\prime}\right\|$, then $Q M \geq Q_{B}^{\prime} M$

Proof. Along the same lines as the proof of Th. 5.16 .

The following theorems are trivial consequences of the preceding.

Theorem 5.20. If $\mathrm{Q}[\mathrm{y}, \mathrm{K}] \mathrm{L} \in \Delta$ and $\mathrm{Q}[\mathrm{y}, \mathrm{K}] \mathrm{L} \geq_{B} \mathrm{Q}\left[\mathrm{y}, \mathrm{K}^{\prime}\right] \mathrm{L}^{\prime}$, then $\mathrm{QK} \geq_{\beta} \mathrm{QK}^{\prime}$.

Theorem 5.21. If $\mathrm{QK} \in \Delta, \mathrm{QK} \geq_{B} \mathrm{Q}^{\prime} \mathrm{K}^{\prime}$ and $\|\mathrm{Q}\|=\left\|\mathrm{Q}^{\prime}\right\|$, then $Q K \geq_{B} Q K^{\prime} \geq_{B} Q^{\prime} K^{\prime}$ and $Q K \geq_{\beta} Q^{\prime} K \geq_{B} Q^{\prime} K^{\prime}$.

We define the beta-equivalence relation as follows:

Definition 5.22. Let $A$ and $B \in \Delta$. We call $A$ beta-equivalent to $B$ (denoted: $A \sim_{B} B$ ) if there is an expression $C$ such that $A \geq_{B} C$ and $B \geq_{B} C$.

It is clear that beta-equivalence is reflexive and symetric. The transitivity of beta-equivalence will be proved in Th. 7.35, using Th. 6.43 (in the literature the transitive closure of betaequivalence is called beta-conversion).

Theorem 5.23. Let $\mathrm{QK}$ and $\mathrm{QL} \in \Delta$. If $\mathrm{QK} \sim_{\beta} \mathrm{QL}$, there is an $\mathrm{N}$ such that $Q K \geq_{\beta} Q N$ and $Q L \geq_{\beta} Q N$. 
Proof. Since $Q K \sim_{\beta} Q L: Q K \geq_{\beta} A$ and $Q L \geq_{\alpha} A$. From Th. 5.12 it follows that $A \equiv Q^{\prime} N$ with $\|Q\|=\left\|Q^{\prime}\right\|$. Then from Th. 5.21: $Q K \geq_{B} Q N$ and $Q L \geq_{B} Q N$.

From this theorem, together with Th. 5.15, it easily follows that the monotony rules hold for beta-equivalence (parts (a), (c) and (d) of the following theorem); part (b) follows from Th. 5.23 and Th. 5.16:

Theorem 5.24.

(a) If $Q C, Q D, Q\{A\} C, Q\{A\} D \in \Delta$ and $Q C \sim_{\beta} Q D$, then $Q\{A\} C \sim_{\beta} Q\{A\} D$.

(b) If $Q C, Q D, Q[x, A] C, Q[x, A] D \in \Delta$ and $Q C \sim_{B} Q D$, then $Q[x, A] C \sim B Q Q[x, A] D$.

(c) If $Q A, Q B, Q\{A\} C, Q\{B\} C \in \Delta$ and $Q A \sim_{B} Q B$, then $Q\{A\} C \sim_{B} Q\{B\} C$.

(d) If $Q A, Q B, Q[x, A] C, Q[x, B] C \in \Delta$ and $Q A \sim_{B} Q B$, then $Q[x, A] C \sim_{B} Q[x, B] C$.

§ 6. OTHER B-REDUCTIONS

In a $B$-reduction we eliminate a pair of the form $\{A\}[x, B]$ occurring in an expression, obtaining "copies" of A (to be precise: expressions $A^{\prime}, A^{\prime \prime}$, etc., which are renovations of $A$ ) instead of the non-binding $x^{\prime} s$ in that expression. We sometimes wish to retain information concerning "past" $\beta$-reductions, as a kind of "scar" in an expression.

The easiest way to do this is to maintain the pair $\{A\}[x, B]$ in an expression after $\beta$-reduction. We shall formalize this kind of $\beta$-reduction, calling it $\beta_{1}$-reduction. Another B-reduction, called $B_{2}$-reduction, will be introduced especially to eliminate the "scars" $\{A\}[X, B]$ as soon as they are no longer required. We shall show that a $\beta$-reduction can be decomposed into a $\beta_{1}$-reduction and a $B_{2}$-reduction.

We describe in this section $\beta_{1}$ - and $\beta_{2}$-reduction as a preparation for Section III.3. 
The fact that we wish to keep the pair. $\{A\}[x, B]$ in an expression after $B_{1}$-reduction complicates matters since we wish each sequence of $\beta$-reductions to have a corresponding. sequence of $\beta_{1}-$ reductions. For example, a sequence of $\beta$-reductions $Q\{A]\{B\}[x, \tau][y, x] y \geq_{B}^{\prime} Q\{A\}[y, F r B] y \geq_{B}^{\prime} Q F r A$ should. have its counterpart in $B_{1}$-reductions: $Q\{A\}\{B\}[x, \pi][y, x] y \geq_{\beta}^{\prime}$ $Q\{A\}\{B\}[x, \tau][y, F r B] y z_{B}, Q\{A\}\{B\}[x, \tau][y, F r B]$ FrA. Note that in the latter single-step $\beta_{1}$-reduction we have to ignore the scar $\{B\}[x, \tau]$ located between $\{A\}$ and $[y, F r B]$ (a property of such a scar $[B\}[x, \tau]$ is that the $x$ does not occur in the expression following it).

However, $B_{1}$-reduction permits more. One may ignore the pair $\{B\}[x, \tau]$ in $Q\{A\}\{B\}[x, \tau][y, x] y$, in spite of the fact that $x$ does occur in $[y, x] y$. This gives the single-step $\beta_{1}$-reduction: $Q\{A\}\{B\}[x, \tau][y, x] y \geq_{B}^{\prime} Q\{A\}\{B\}[x, \tau][y, x]$ FrA, by applying $[y, x] y$ to $A$. This is a real extension of the usual B-reduction concept. We shall call a string like $\{B\}[x, \tau]$ in the above example, which may be $10-$ cated between "function" and "argument" of a $\beta_{1}$-reduceable expression, a B-chain.

Moreover, we shall agree that the relation $Q\{A\} P[y, C] D \geq_{B}^{\prime} Q\{A\} P[y, C](y:=A) D$ (in which $P$ is a B-chain) does only hold if $y^{1}$ occurs in $D$. If we did not require this, one could continue the $\beta_{1}$-reduction with the latter expression, thus producing a non-terminating $B_{1}$-reduction sequence.

The $B_{2}$-reduction relation, on the other hand, eliminates an applicator and an abstractor as in $Q\{A\} P[y, C] E . \geq_{B_{2}}^{\prime} Q P E$ (in which $P$ is again a $B$-chain); the latter relation only holds, however, if $\mathrm{y}$ does not occur in $\mathrm{E}$.

We give an inductive definition of $\beta$-chain:

Definition 6.1.

(1) If $P \equiv \phi$, then $P$ is a $\beta$-chain.

(2) If $P$ is a $B-c h a i n$, then $\{B\} P[X, C]$ is a $B$-chain.

(3) If $P_{1}$ and $P_{2}$ are $\beta$-chains, then $P_{1} P_{2}$ is a $\beta$-chain.

Example: $\{A\}\{B\}[x, C]\{D\}[y, E][z, E]$ is a $B$-chain. 
Notation Rule 6.2. We write $\hat{\mathrm{P}}$ to indicate that $\mathrm{P}$ is a B-chain. We write $[\dot{x}, B] C$ to indicate in $[x, B] C$ that $x \notin C$.

A $B$-chain $\hat{P}$ has the property that the number of applicators in $P$ is equal to the number of abstractors in $P$. Moreover, if $\hat{P} \equiv P_{1} P_{2}$, the number of applicators in $P_{1}$ is at least equal to the number of abstractors in $\mathrm{P}_{1}$.

We can also express this by means of a valuation $v$ of lambdaphrase chains, defined inductively by (1) $\mathrm{v}(\phi)=0$,

(2) $v(\{A\} P)=v(P)+1,(3) v([x, A] P)=v(P)-1$.

Then for a $B$-chain $\hat{P}$ it holds that $(i) v(\hat{P})=0$, and (ii) if $\hat{\mathrm{S}} \equiv \mathrm{P}_{1} \mathrm{P}_{2}$, then $\mathrm{v}\left(\mathrm{P}_{1}\right) \geq 0$. These conditions are also sufficient. The following theorem concerning B-chains can be proved by the aid of the above-mentioned valuation properties :

Theorem 6.3.

(1) If $\widehat{\mathrm{P}} \phi$, then $\widehat{\mathrm{P}} \equiv \mathrm{P}^{\prime}\{\mathrm{B}\}[\mathrm{x}, \mathrm{C}] \mathrm{P}^{\prime \prime}$.

(2) $P \equiv P_{1} P_{3}$ is a $B$-chain if and only if $P^{\prime} \equiv P_{1} \widehat{P}_{2} P_{3}$ is a B-chain.

Note that for each $\hat{\mathrm{P}} \phi$ there is a unique decomposition $\hat{\mathrm{P}} \equiv \hat{\mathrm{P}}_{1} \hat{\mathrm{P}}_{2} \ldots \hat{\mathrm{P}}_{\mathrm{n}}$ with $\hat{\mathrm{P}}_{i} \not \phi$ and $\hat{\mathrm{P}}_{i} \neq \hat{\mathrm{P}}_{i} \hat{\mathrm{P}}_{i}^{\prime \prime}$ for some $\mathrm{P}_{i}^{\prime} \neq$ and $P_{i}^{\prime \prime} \not$.

The following theorem shows that a $\beta$-chain has a compact structure:

Theorem 6.4. If $\{A\} \hat{P}[x, B] C \subset \widehat{P}, F$, then either $\{A\} \hat{P}[x, B]$ is a part of $\hat{\mathrm{P}}_{1}$ (i.e. $\hat{\mathrm{P}}_{1} \equiv \mathrm{S}_{1}\{\mathrm{~A}\} \hat{\mathrm{P}}[\mathrm{x}, \mathrm{B}] \mathrm{S}_{2}$ ), or $\{\mathrm{A}\} \hat{\mathrm{P}}[\mathrm{x}, \mathrm{B}] \mathrm{C} \subset \mathrm{F}$.

Proof. The essential part of the theorem is that the following cannot occur: $P \equiv P_{2} P_{3}, P_{1} \equiv P_{4}\{A\} P_{2}$ and $F \equiv P_{3}[x, B] C$ (" $\{A\} \hat{P}[x, B] C$ occurs partially in $P_{1}$, partially in $\left.F^{\prime \prime}\right)$. This can be proved by the aid of the valuation properties for B-chains.

We continue with the definitions of single-step $\beta_{1}-$ and $B_{2}-$ reduction: 
Definition 6.5. Single-step $\beta_{1}$-reduction is the relation generated by

(1) If $Q\{A\} \hat{P}[x, B] C \in \Delta$ and $x \in C$, then $Q\{A\} \hat{P}[x, B] C \geq_{B}^{\prime}$ Q\{A\} $\hat{P}[x, B](x:=A) C$, and (2) the monotony rules (see Def. $5.5(2),(3)$ and (4), reading $\geq_{\beta}^{\prime}$
instead of $\geq_{\beta}^{\prime}$ ).

Definition 6.6. $\beta_{1}$-reduction is the reflexive and transitive closure of single-step $B_{1}$-reduction.

Definition 6.7 . Single-step $B_{2}$-reduction is the relation generated by

(1) If $Q\{A\} \hat{P}[x, B] C \in \Delta$, then $Q\{A\} \hat{P}[x, B] C z_{B_{2}}^{\prime} Q \hat{P} C$, and

(2) the monotony rules.

Definition 6.8. $\beta_{2}$-reduction is the reflexive and transitive closure of single-step $\beta_{2}$-reduction.

Note that in Def. $6.7 \mathrm{x}$ may not occur in $\mathrm{C}$.

As in the case of $B$-reduction (see the previous section), we speak of elementary $\beta_{1}$ - or $\beta_{2}$-reduction, $n$-step $\beta_{1}-$ or $\beta_{2}$-reduction, and a single-step $B_{1}$ - or $B_{2}$-reduction generated by $Q\{A\} \hat{P}[x, B] C$ or $Q\{A\} \hat{P}[\stackrel{\circ}{B}, B] C$ respectively. The "length of proof of $\mathrm{K} \geq_{B_{1}}^{\prime} \mathrm{L}$ " or "... K $\geq_{B_{2}}^{\prime} \mathrm{L}$ " is defined as for single-step $B$-reduction. Finally we define $\beta_{1}$-equivalence $\left(K \sim_{B_{1}} L\right)$ and $\beta_{2}$-equivalence $\left(K \sim_{B_{2}} L\right.$ ) analogously to $\beta$-equivalence (see ${ }^{1}$ the previous section).

Theorem 6.9. If $K \in \Delta$ and $K \geq_{\beta_{1}}^{\prime} L$ or $K \geq_{B_{2}}^{\prime} L$, then $L \in \Delta$. Proof. Induction on the length of proof of $\mathrm{K} \geq_{\beta_{1}}^{\prime} \mathrm{L}$ or $K \geq_{\beta_{2}}^{\prime} L$ respectively. Cf. the proof of Th. 5.8 .

Definition 6.10. We shall write $P \sum_{B_{1}} P^{\prime}$ etc. if $P \tau z_{\beta_{1}} P^{\prime} \tau$ etc. 
Theorem 6.11. If $Q A \in \Delta$ and $Q A \geq{ }_{B}^{\prime} K$ (or $Q A \geq{ }_{B}^{\prime} K$ ), then $K \equiv Q^{\prime} A^{\prime}$ with $\|Q\|=\left\|Q^{\prime}\right\|$, and either

(1) $Q \geq_{B_{1}}^{\prime} Q^{\prime}$ (or $Q \geq_{B_{2}}^{\prime} Q^{\prime}$, respectively) and $A \equiv A^{\prime}$, or

(2) $Q \equiv Q^{\prime}$ and $Q A \geq_{B}^{\prime} Q^{\prime}$ (or $Q^{\prime} \geq_{B_{2}}^{\prime} Q^{\prime}$ respectively).

Proof. Cf. the proofs of Th, 5.11 and Th. 5.12.

Theorem 6.12. The monotony rules hold for $\beta_{1}$-reduction and for $\beta_{2}{ }^{-}$ reduction.

Proof. Cf, the proof of Th. 5.15 .

Theorem 6.13. If $Q C, Q P C$ and $Q P D e \Delta$, and $Q C \geq_{\beta_{1}} Q D$ (or $Q C \geq_{\beta_{2}} Q D$ ), then $\mathrm{QPC} \geq_{\beta_{1}} \mathrm{QPD}$ (or $\mathrm{QPC} \geq_{\beta_{2}} \mathrm{QPD}$ respectively).

Proof. Cf, the proof of Th. 5.17 .

The following two theorems deal with the relation between $B-$ reduction on the one hand and $\beta_{1}$ - and $\beta_{2}$-reduction on the other.

Theorem 6.14 . If $K \geq_{B}^{\prime} L$, then $K \sim_{B} L$.

Proof. Induction on the length of proof of $K \geq_{B}^{\prime}$.

I. $K z_{B_{1}}^{\prime}$ I is $Q\{A\} \hat{P}[x, B] C \geq_{B}^{*} Q\{A\} \hat{P}[\hat{x}, B](x:=A) C$.

Note that, if $\widehat{P} \not \phi$, then $\hat{P} \equiv P^{\prime}\{B\}[x, C] P^{\prime \prime}$, and $P_{1} \equiv P^{\prime} P^{\prime \prime}$ is again a $\beta$-chain by $T h .6 .3$. It follows that there is a $\beta$-reduction for $K$ and for $L$. Continuation of this $\beta$-reduction process gives: $K \geq_{B} Q\{A\}\left[x, B^{\prime}\right] C^{\prime} \geq_{B} Q(x:=A) C^{\prime}$, and $I \geq_{B} Q\{A\}[x, B](x:=A) C^{\prime} z_{B}$ $Q(x:=A) C^{\prime}$. In the latter reduction one should note that the substitutions $(y:=D)$ introduced in the reduction $L{ }_{B} Q\{A\}[x, B](x:=A) C^{\prime}$ do not influence $A$, since $y \notin A$. Together with the statement that in this case $(y:=D)(x:=A) E \equiv(x: m A)(y:=D) E$ (but for renaming) this results in us obtaining the same $C^{\prime}$ (but for renaming) in reducing $L$ as we obtained in reducing $K$. It follows that $K \geq_{B} L$. 
II. $K \geq_{B_{1}}^{\prime} L$ is $Q\{A\} C \geq_{B_{1}}^{\prime} Q\{A\} D$ as a direct consequence of $Q C \geq_{B_{1}}^{\prime} Q D$. Then, by induction: $Q C \sim_{\beta} Q D$. Hence, by Th. 5.24: $Q\{A\} C \sim_{\beta} Q\{A\} D$. III, IV. The two other monotony cases are proved similarly to II.

Theorem 6.15. If $\mathrm{K} \in \Delta$ and $\mathrm{K} \geq_{B}^{\prime} \mathrm{L}$, then $\mathrm{K} \geq_{B_{1}^{\prime}}^{\prime} \geq_{B_{2}}^{\prime} \mathrm{L}$ or $\mathrm{K} \geq_{B_{2}}^{\prime} \mathrm{L}$.

Proof. Let $K \geq_{B}^{\prime}$ L be generated by $Q\{A\}[x, B] C \geq_{B}^{\prime} Q(x:=A) C$. If $x \in C$, then $Q\{A\}[x, B] C \geq_{B_{1}}^{\prime} Q\{A\}[x, B](x:=A) C \geq_{\beta_{2}}^{\prime} Q(x:=A) C$. If $x \notin C$, then $K \geq_{\beta}^{\prime} L$ is a $\beta_{2}$-reduction. The remainder follows from the fact that the monotony rules for $B_{-}, B_{1}-$ and $B_{2}$-reduction are similar.

We shall now prove a number of theorems leading to a theorem on the possibility of postponement of $\beta_{2}$-reductions after $\beta_{1}-$ reductions (Th. 6.19 ).

Theorem 6.16. If $K \in \Delta, K \geq_{B_{2}}^{\prime} L \geq_{B_{1}}^{\prime} M$, then $K \geq_{\beta_{1}}^{\prime} L^{\prime} \geq_{B_{2}}^{n} M$ for a certain $n \geq 1$.

Proof. Let $L \geq_{B_{1}^{\prime}}^{\prime} M$ be generated by $Q\{A\} \hat{P}[x, B] C \geq_{\beta_{1}}^{\prime} Q\{A\} \hat{P}[x, B](x:=A) C$, and $K \geq_{B_{2}}^{\prime} L$ by $Q^{\prime}\{D\} \hat{\mathrm{P}}_{1}\left[{ }^{0}, E\right] F \geq_{B_{1}}^{\prime} Q^{\prime} \hat{\mathrm{P}}_{1} F$. Then $\{A\} \hat{P}[x, B] C \subset L$ and $\hat{\mathrm{P}}_{1} \mathrm{~F} \subset \mathrm{L}$. ${ }^{2}$ Now we can distinguish three cases: (1) $\{\mathrm{A}\} \hat{\mathrm{P}}[\mathrm{x}, \mathrm{B}] \mathrm{C}$ and $\hat{\mathrm{P}}_{1} \mathrm{~F}$ occur in $\mathrm{L}$ in disjoint places, (2) $\{\mathrm{A}\} \hat{\mathrm{P}}[\mathrm{x}, \mathrm{B}] \mathrm{C}=\hat{\mathrm{P}}_{1} F$, $\hat{\mathrm{P}}_{1} \mathrm{~F} \subset\{\mathrm{A}\} \hat{\mathrm{P}}[\mathrm{x}, \mathrm{B}] \mathrm{C}$ and $\hat{\mathrm{P}}_{1} \mathrm{~F} \not\{\{\hat{\mathrm{P}}[\mathrm{P}, \mathrm{B}] \mathrm{C}$.

(1) In this case it is clear that the theorem holds for $\mathfrak{n}=1$.

(2) We may distinguish (see also Th. 6.4):

(a) $\hat{\mathrm{P}}_{1} \equiv \mathrm{P}_{2}\{\mathrm{G}\} \mathrm{P}_{3}$ or $\hat{\mathrm{P}}_{1} \equiv \mathrm{P}_{2}[\mathrm{z}, \mathrm{G}] \mathrm{P}_{3}$ and $\{\mathrm{A}\} \hat{\mathrm{P}}[\mathrm{x}, \mathrm{B}] \mathrm{C}=\mathrm{G}$. The theorem holds for $\mathrm{n}=1$.

(b) $\hat{\mathrm{P}}_{1} \equiv \mathrm{P}_{2}\{A\} \hat{\mathrm{P}}[\mathrm{x}, \mathrm{B}] \mathrm{P}_{3}$. Idem.

(c) $\{A\} \hat{P}[x, B] C \subset F$. Idem.

These cases $(a)-(c)$ are exhaustive if $\{A\} \hat{P}[x, B] C \subset \hat{P}_{1} F$.

(3) (a) Let $\hat{P}_{1} F \subset A$. Now the theorem holds for $n$ is the number of occurrences of $x$ in $C$ plus one. 
(b) Let $\hat{\mathrm{P}} \equiv \mathrm{P}_{2} \hat{\mathrm{P}}_{1} \mathrm{P}_{3}$. The theorem holds for $\mathrm{n}=1$.

(c) Let $\hat{\mathrm{P}} \equiv \mathrm{P}_{2}[\mathrm{G}] \mathrm{P}_{3}$ or $\hat{\mathrm{P}} \equiv \mathrm{P}_{2}[\mathrm{z}, \mathrm{G}] \mathrm{P}_{3}$ and $\hat{\mathrm{P}}_{1} \mathrm{~F} \subset \mathrm{G}$. Idem.

(d) Let $\hat{\mathrm{P}}, \mathrm{F} \in[\mathrm{x}, \mathrm{B}] \mathrm{C}$. Idem.

These cases (a)-(d) are exhaustive if $\hat{\mathrm{P}}_{1} \mathrm{~F} \subset\{\mathrm{A}\} \hat{\mathrm{P}}[\mathrm{x}, \mathrm{B}] \mathrm{C}$ and $\hat{\mathrm{P}}_{1} F \neq\{\mathrm{A}\} \hat{\mathrm{P}}[\mathrm{x}, \mathrm{B}] \mathrm{C}$. (In this proof we several times use the lemma: "If $\hat{\mathrm{P}} \equiv \mathrm{P}_{1} \hat{\mathrm{P}}_{2} \mathrm{P}_{3}$, then $\mathrm{P}_{1}\{\mathrm{G}\} \hat{\mathrm{P}}_{2}[\mathrm{z}, \mathrm{H}] \mathrm{P}_{3}$ is also a B-chain", which is a consequence of Th. 6.3.)

Theorem 6.17. If $K \in \Delta, K \geq_{B_{2}} L \geq_{B_{1}^{\prime}}^{\prime} M$, then $K \geq_{B_{1}}^{\prime} L^{\prime} \geq_{B_{2}} M$.

Proof. Induction on the number of steps of $\mathrm{K} \geq_{B_{2}} \mathrm{~L}$, using Th. 6.16 . Theorem 6.18. If $\mathrm{K} \in \Delta, \mathrm{K} \geq_{B_{2}} \mathrm{~L} \geq_{B_{1}}^{\mathrm{P}} \mathrm{M}$, then $\mathrm{K} \geq \frac{\mathrm{P}}{\mathrm{B}_{1}} \mathrm{~L}^{\prime} \geq_{B_{2}} \mathrm{M}$. Proof. Induction on p, using Th. 6.17 .

Theorem 6.19. If $\mathrm{k}_{1} \geq^{\prime} \mathrm{k}_{2} \geq^{\prime} \ldots \geq^{\prime} \mathrm{k}_{\mathrm{n}}$ by single-step $\beta_{1}-$ and $\beta_{2}-$ reductions, the total number of $B_{1}$-reductions being $p$, there is a reduction $K_{1} \equiv I \geq \sum_{B_{1}}^{P} M \geq_{B_{2}} N \equiv K_{n}$.

Proof. Combine the successive single-step $B_{1}$-reductions in $\mathrm{K}_{1} \geq^{\prime} \mathrm{K}_{2} \geq^{\prime} \ldots \geq^{\prime} \mathrm{K}_{\mathrm{n}}$, and do the same with the successive singlestep $B_{2}$-reductions: we obtain $K_{1} \equiv L_{1} \geq{ }_{B_{2}} M_{1} \geq_{\beta_{1}} L_{2} \geq_{B_{2}} M_{2} \geq_{B_{1}} \ldots$ $\geq_{B_{1}} L_{\ell} \geq_{B_{2}} M_{\ell} \geq_{\beta_{1}} K_{n}$. Induction on $\ell$ yields ${ }^{2}$ the proof.

We shall now prove what we call the Church-Rosser propenty (CR) for $\beta_{1}$-reduction, which we formulate as follows: If $K \geq_{B} L$ and $K \geq_{B} M$, there is an $N$ such that $L \geq_{B} N$ and $M \geq_{B} N$. We can express this Church-Rosser property in a diagram, as follows:

CR :

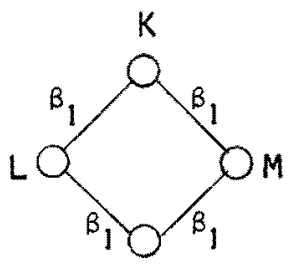


From $C R$ for $\beta_{1}$-reduction it easily follows that $\beta_{1}$-equivalence is transitive, hence indeed an equivalence relation (reflexivity and symetry of $\sim_{B_{1}}$ are trivial). Hence we can also state that $\sim_{B_{1}}$ is the equivalence relation generated by $\geq_{B_{1}}$, which is an alternative formulation for the Church-Rosser theorem for $\beta_{1}$-reduction.

In proving $C R$ for $\beta_{1}$-reduction we shall use a technique introduced by W.W. Tait and P. Martin-Löf, given in Barendregt [1, appendix II]. We shall discuss the power of this technique in brief.

In order to prove $C R$ for a reduction it is natural to begin with single-step reductions " $K \geq{ }^{\prime} L$ " and " $K \geq ' M^{\prime \prime}$. In a usual singlestep (e.g. $\beta-$ ) reduction one can then find an $N$ such that $" L \geq N$ " and $" M \geq N "$, but unfortunately only one of these last two reductions is necessarily a single-step reduction, and one cannot say in advance which of the two.

If one now begins with multiple-step reductions $" K \geq L "$ and " $K \geq M "$ and one tries, by the aid of the above, to find an $N$ such that " $L \geq N$ " and $" M \geq N$ ", then the termination of this attempt is not guaranteed. The following example, drawn in a diagram, suggests what might happen:

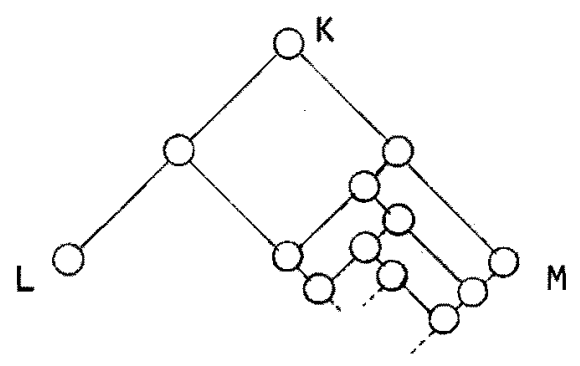

Bach rectangle in this diagram represents reductions; three sides of the rectangle are single-step reductions, one side is twostep. The diagram can, however, be continued indefinitely in the place where we draw the dotted lines. 
Now Tait and Martin-Löf defined, a new "single"-step reduction (which we shall call single-step nested reduction to avoid confusion). The latter reduction has the property that with each pair of single-step nested reductions " $\mathrm{K} \geq{ }^{*} \mathrm{~L}^{\text {" and }}$ " $\mathrm{K} \geq{ }^{\star} \mathrm{M}^{\text {" there can be }}$ found an $N$ such that "L $\geq^{*} N^{\prime}$ and "M $\geq^{*} N$ ", both last-mentioned reductions being single-step nested reductions as wel1. Moreover, each multiple-step reduction can be decomposed into single-step nested reductions and each single-step nested reduction is a composition of (ordinary) single-step reductions.

If one now begins with multiple-step reductions " $\mathrm{K} \geq \mathrm{L}$ " and " $\mathrm{K} \geq \mathrm{M}$ ", one can decompose these reductions into single-step nested reductions and apply the above. Then one obtains, for example, a situation as is expressed in the following diagram:

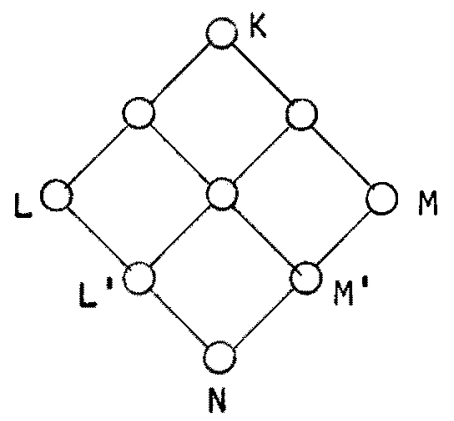

In each of these restangles the four sides represent singlestep nested reductions.

Moreover, the nested reductions " $\mathrm{L} \geq^{*} \mathrm{~L}$ ' $\geq^{*} \mathrm{~N}$ " and " $M \geq^{*} M^{\prime} \geq^{*} N^{\prime \prime}$ can be decomposed into (ordinary) single-step reductions, which combine into " $L \geq N$ " and "M $\geq N$ ". Thus we obtain $C R$.

In the following we shall define a single-step nested $\beta_{1}$ reduction, which we shall call single-step $\gamma$-reduction. Our $\gamma-$ reduction is a little more complex than the nested reduction of Tait and Martin-Löf, but it yields essentially the same results.

The "nested" character of $\gamma$-reduction can be explained as follows. Let a $\beta_{1}$-reduction be generated by $Q[A] \widehat{P}[x, B] C$, let 
$Q A \geq \geq_{\gamma}^{\prime} Q A^{\prime}$ and $Q \hat{P}[x, B] C \geq_{\gamma}^{\prime} Q^{\prime} \hat{P}^{\prime}\left[x, B^{\prime}\right] C^{\prime}$. Then also $Q\{A\} \hat{P}[x, B] C \geq_{\gamma}^{\prime}$ $Q\left\{A^{\prime}\right\} \hat{P}^{\prime}\left[x, B^{\prime}\right]\left(x:=A^{\prime}\right) C^{\prime}$ (if the suggested $B_{1}$-reduction is preceded by single-step nested reductions "inside" $A, P, B$ and $C$, the composite reduction is a single-step nested reduction). The reductions take place in a "nested" order.

With the aid of $\gamma$-reduction we shall prove $C R$ for $\beta_{1}$-reduction. We shall subsequently prove $C R$ for $\beta$-reduction, using $C R$ for $\beta_{1}$ reduction (we could also prove $C R$ for B-reductions directly, along the same lines pointed out by Tait and Martin-Löf).

Definition 6.20. Single-step $\gamma$-reduction, denoted by $z_{\gamma}^{\prime}$, is the reflexive relation generated by

(1) If $Q\{A\} \hat{P}[x, B] C \in \Delta, x \subset C, Q A \geq_{\gamma}^{\prime} Q A^{\prime}$ and $Q \hat{P}[x, B] C \geq_{\gamma}^{\prime}$ $\mathrm{Q} \hat{\mathrm{P}}^{\prime}\left[\mathrm{X}, \mathrm{B}^{\prime}\right] \mathrm{C}^{\prime}$, then $Q\{A\} \hat{P}[x, B] C \geq_{\gamma}^{\prime} Q\left\{A^{\prime}\right\} \hat{P}^{\prime}\left[x, B^{\prime}\right]\left(x:=A^{\prime}\right) C^{\prime}$.

(2) If $Q\{A\} C$ and $Q\left\{A^{\prime}\right\} C^{\prime} \in \Delta, Q A z_{\gamma}^{\prime} Q A^{\prime}$ and $Q \dot{C} \geq_{\gamma}^{\prime} Q C^{\prime}$, then $Q\{A\} C \geq_{\gamma}^{\prime} Q\left\{A^{\prime}\right\} C^{\prime}$.

(3) If $Q[x, A] C$ and $Q\left[x, A^{\prime}\right] C^{\prime} \in \Delta, Q A \geq_{\gamma}^{\prime} Q A^{\prime}$ and $Q[x, A] C \geq_{\gamma}^{\prime} Q[x, A] C^{\prime}$, then $Q[x, A] C \geq_{Y}^{\prime} Q\left[x, A^{\prime}\right] C^{\prime}$.

(4) If $A \in \Delta, A \geq \geq_{\gamma}^{\prime} B$ and $B \geq{ }_{\alpha} B^{\prime}$, then $A \geq_{\gamma}^{\prime} B^{\prime}$.

We call (2) and (3) the monotony mites for single-step $\gamma-$ reduction. We also define $\gamma$-equivalence $\left(K \sim_{\gamma} L\right)$ analogously to $B-$ equivalence.

Definition 6.21. $\gamma$-reduction, denoted by $\geq_{\gamma}$, is the transitive closure of single-step $\gamma$-reduction.

We continue with some theorems concerning $\gamma$-reduction (it will be clear that $Q \geq_{\gamma}^{\prime} Q^{\prime}$ if and only if $\left.Q \tau \geq_{\gamma}^{\prime} Q^{\prime} \tau\right)$.

Theorem 6.22. If $Q A \in \Delta$ and $Q A \geq_{Y}^{\prime} K$, then $K \equiv Q^{\prime} A^{\prime}$, where $\|Q\|=\left\|Q^{\prime}\right\|, Q \geq \geq_{\gamma}^{\prime} Q^{\prime}$ and $Q A \geq_{\gamma}^{\prime} Q A^{\prime}$.

Proof. Induction on the length of proof of $Q A \geq_{\gamma}^{\prime} K$.

(1) If $\mathrm{QA} \geq_{\gamma}^{\prime} \mathrm{K}$ by reflexivity, the theorem is trivial.

(2) If $Q A \geq_{\gamma}^{\prime} K$ is $Q_{0}\{B\} \hat{P}[x, C] D \geq_{\gamma}^{\prime} Q_{0}\left\{B^{\prime}\right\} \hat{P}^{\prime}\left[x, C^{\prime}\right]\left(x:=B^{\prime}\right) D^{\prime}$ as a 
direct consequence of $Q_{0} B_{\gamma}^{\prime} Q_{0} B^{\prime}$ and $Q_{0} \hat{P}[x, C] D \geq_{\gamma}^{\prime} Q_{0} \hat{P}^{\prime}\left[x, C^{\prime}\right] D^{\prime}$, then $\|Q\| \leq\left\|Q_{0}\right\|$, so $Q_{0} \equiv Q Q^{\prime \prime}$, and the theorem follows.

(3) If $Q A \geq \geq_{\gamma}^{\prime} K$ is $Q_{0}\{B\} C \geq_{\gamma}^{\prime} Q_{0}\left\{B^{\prime}\right\} C^{\prime}$ as a direct consequence of $Q_{0} B \geq{ }_{\gamma}^{\prime} Q_{0} B^{\prime}$ and $Q_{0} C^{\prime} \geq_{\gamma}^{\prime} Q_{0} C^{\prime}$, then again $\|Q\| \leq\left\|Q_{0}\right\|$ and the theorem follows.

(4) Let $Q A \geq \geq_{\gamma}^{\prime} K$ be $Q_{0}[x, B] C \geq_{\gamma}^{\prime} Q_{0}\left[x, B^{\prime}\right] C^{\prime}$ as a direct consequence of $Q_{0} B \geq{ }_{\gamma}^{\prime} Q_{0} B^{\prime}$ and $Q_{0}[x, B] C \geq \geq_{\gamma}^{\prime} Q_{0}[x, B] C^{\prime}$.

(i) If $\|Q\| \leq\left\|Q_{0}\right\|$ or $Q \equiv Q_{0}[x, B]$ then the theorem follows. (ii) If $C \equiv\left[y_{1}, B_{1}\right] \ldots\left[y_{n}, B_{n}\right] C_{0}$ and $Q \equiv Q_{0}[x, B]\left[y_{1}, B_{1}\right] \ldots$ $\left[y_{n}, B_{n}\right]$, then $Q A \equiv Q_{0}[x, B] C \equiv Q_{0}[x, B]\left[y_{1}, B_{1}\right] \ldots\left[y_{n}, B_{n}\right] A \geq \gamma$ $Q_{0}[x, B] C^{\prime} \equiv Q_{0}[x, B]\left[z_{1}, B_{1}^{\prime}\right] \ldots\left[z_{n}, B_{n}^{\prime}\right] A^{\prime}$ (by induction) with $Q A \geq_{\gamma}^{\prime} Q A^{\prime}$ and $Q \geq_{\gamma}^{\prime} Q_{0}[x, B]\left[z_{1}, B_{j}^{\prime}\right] \ldots\left[z_{n}, B_{n}^{\prime}\right]$.

It follows that $Q \geq_{\gamma}^{\prime} Q_{0}\left[x, B^{\prime}\right]\left[z_{1}, B_{i}^{\prime}\right] \ldots\left[z_{n}, B_{n}^{\prime}\right]$. Also $C^{\prime} \equiv\left[z_{1}, B_{1}^{\prime}\right] \ldots\left[z_{n}, B_{n}^{\prime}\right] A^{\prime}$, so $Q_{0}\left[x, B^{\prime}\right] C^{\prime} \equiv$ $Q_{0}\left[x, B^{\prime}\right]\left[z_{1}, B_{1}^{\prime}\right] \ldots\left[z_{n}, B_{n}^{\prime}\right] A^{\prime}$. Consequently the theorem holds if we take $Q^{\prime} \equiv Q_{0}\left[x, B^{\prime}\right]\left[z_{1}, B_{1}^{\prime}\right] \ldots\left[z_{n}, B_{n}^{\prime}\right]$.

(5) Let $Q A \geq_{\gamma}^{\prime} K$ be a direct consequence of $Q A \quad \geq_{\gamma}^{*} K^{\prime}$ and $K^{\prime} \geq_{\alpha} K$. Then by induction the theorem holds for $Q A \geq_{\gamma}^{\prime} K^{\prime}$, and trivially al so for $Q A \geq_{\gamma}^{\prime} K$.

Theorem 6.23. The monotony rules hold for $y$-reduction.

Proof. Cf. the proof of Th. 5.15.

Theorem 6.24. If $Q C, P C$ and $P D \in \Delta$, and $Q C z_{\gamma}^{\prime} Q D$, chen $P C z_{\gamma}^{\prime} P D$. Proof. Analogous to the proof of Th, 5.17.

The following two theorems deal with the relation between $\beta_{1}$ and $\gamma$-reduction.

Theorem 6.25. If $K \in \Delta$ and $K \geq_{B_{1}}^{\prime} L$, then $K \geq_{\gamma}^{\prime} L$.

Proof. Induction on the length of proof of $K \geq_{\beta_{1}}^{\prime}$. The rule of elementary $\beta_{1}$-reduction is covered by Def. 6a.11(1) (take $Q A \geq_{\gamma} Q A^{\prime}$ to be $Q A \geq_{\gamma} Q A$ by reflexivity, etc.), the monotony rules for $B_{1}-$ 
reduction are covered by the monotony rules for $\gamma$-reduction (again using the reflexivity of $\gamma$-reduction in appropriate places).

Theorem 6.26. If $\mathrm{K} \in \Delta$ and $\mathrm{K} \geq_{Y}^{\prime} \mathrm{L}$, then $\mathrm{K} z_{\beta_{1}}$ L but for $\alpha$-reduction.

Proof. Induction on the length of proof of $K \geq_{Y}^{\prime}$ L. For example: let $X \geq_{Y}^{\prime} L$ be $Q\{A\} \hat{P}[x ; B] C \geq_{Y}^{\prime} Q\left\{A^{\prime}\right\} \hat{P}^{\prime}\left[x, B^{\prime}\right]\left(x:=A^{\prime}\right) C^{\prime}$ as a direct consequence of $x \in C, Q A \geq A_{Y}^{\prime} Q A^{\prime}$ and $Q P[x, B] C \geq_{Y}^{\prime} Q P^{\prime}\left[x, B^{\prime}\right] C^{\prime}$. By induction the last two reductions can also be obtained by $B_{1}$ reductions and $\alpha$-reductions, and $Q\{A\} \hat{P}[x, B] C \geq_{\beta_{1}} Q\{A\} \hat{P}^{\prime}\left[x, B^{\prime}\right] C^{\prime} \geq_{\beta_{1}}$ $Q\left\{A^{\prime}\right\} \hat{P}^{\prime}\left[x, B^{\prime}\right] C^{\prime} \geq_{B_{1}} Q\left\{A^{\prime}\right\} \hat{P}^{\prime}\left[x, B^{\prime}\right]\left(x:=A^{\prime}\right) C^{\prime}$ (but for $\alpha$-reduction). In the last $\beta_{1}$-reduction we use the lemma: "If $x \subset C$, if $x$ occurs as a binding variable in $Q_{1}$ and if $Q_{1} C \geq_{B_{1}} Q_{1} C^{\prime}$, then $x \subset C^{\prime}$ ".

Theorem 6.27. If $K \in \Delta$ and $K \geq_{Y} L$, then $L \leqslant \Delta$.

Proof. Follows from Th. 6.26 and Th. 6.9.

We inductively define simizarity of two lambda-phrase chains (not necessarily B-chains):

Definition 6.28.

(1) If $P_{1} \equiv P_{2} \equiv \phi$, then $P_{1}$ and $P_{2}$ are similar.

(2) If $P_{1}$ and $P_{2}$ are similar, then $\{A\} P_{1}$ and $\{B\} P_{2}$ are similar, and $[x, A] P_{1}$ and $[x, B] P_{2}$ are similar.

The following theorems are a preparation for Th. 6.37, which expresses $C R$ for $\gamma$-reduction. In order to prove Cor, 6.31 and $T h$. 6.34 it is convenient to extend the notion of $\beta$-chain, as in Def. 6.29: a number of $\beta$-chains, connected by abstractors, will be called a $B$-chain complex. A $\beta$-chain complex may be empty.

Definition 6.29. Let $\mathrm{P}_{1}, \mathrm{P}_{2}, \ldots, \mathrm{P}_{i}$ be (possibly empty) B-chains. Then a lambda chain $P_{1}\left[x_{1}, A_{1}\right] P_{2}\left[x_{2}, A_{2}\right] \ldots P_{i-1}\left[x_{i-1}, A_{i-1}\right] P_{i}$ is called a B-chain complex.

We denote a $\beta$-chain complex $\mathrm{P}$ by $\overline{\overline{\mathrm{P}}}$. 
The following statement can be proved by the aid of valuations: If $P$ is a $\beta$-chain complex and $P \equiv P_{1} P_{2}$, then $P_{2}$ is a $B$-chain complex.

Theorem 6.30. If $Q^{\overline{\bar{P}_{A}}} \in \Delta$ and $\mathrm{Q}_{\overline{\mathrm{P}}} \geq_{\gamma}^{\prime} \mathrm{K}$, then $\mathrm{K} \equiv \mathrm{Q}^{\prime} \overline{\overline{\mathrm{P}^{\prime}}} \mathrm{A}^{\prime}$, where

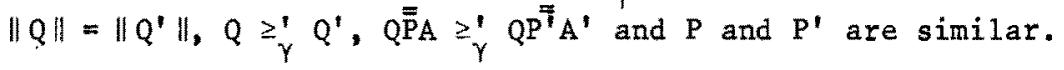

Proof. If $P \equiv Q_{1}$ (including $P \equiv \phi$ ), then $T h$. 6.22 gives the proof. Let $P \neq Q_{1}$. We proceed with induction on the length of proof of $\mathrm{Q} \overline{\overline{\mathrm{P}}} \geq_{\gamma}^{\prime} \mathrm{K}$. Note that there must be at least one applicator in the lambda chain $P$, on account of our assumption $P \neq Q_{1}$.

(1) Assume that $Q \overline{\bar{P}} \bar{A} z_{\gamma}^{\prime} \mathrm{K}$ by reflexivity. The proof is now trivia1.

(2) Assume that $Q \overline{\bar{P} A} z_{\gamma}^{\prime} K$ is $Q Q_{1}\{C\} \hat{P} F[y, D] E z_{Y}^{\prime} Q Q_{1}\left\{C^{\prime}\right\} \hat{P}_{1}^{\prime}\left[y, D^{\prime}\right]\left(y:=C^{\prime}\right) E^{\prime}$ as a direct consequence of $Q Q_{1} C \geq_{\gamma}^{\prime} Q_{1} C^{\prime}$ and $Q Q_{1} \hat{P}_{1}[y, D] E \geq_{\gamma}^{\prime}$ $Q Q_{1} \hat{P}_{1}^{\prime}\left[y, D^{\prime}\right] E^{\prime}$. Now it must hold that $E \equiv P_{2}^{A}$, while $Q_{1} \hat{P}_{1}[y, D] P_{2}$ is a B-chain complex. By induction: $E^{\prime} \equiv \overline{P_{2}^{\prime}} A^{\prime}$ and $P_{2}$ and $P_{2}^{\prime}$ are similar. The remainder is easy.

(3) Assume that $Q \overline{\bar{P}} \geq_{\gamma}^{\prime} K$ is $Q Q_{1}\{C\} D z_{\gamma}^{\prime} Q Q_{1}\left\{C^{\prime}\right\} D^{\prime}$ as a direct consequence of $Q Q_{1} C \geq_{\gamma}^{\prime} Q Q_{1} C^{\prime}$ and $Q Q_{1} D \geq_{\gamma}^{\prime} Q Q_{1} D^{\prime}$. Now $\overline{\bar{P}} \equiv Q_{1}\{C\} \overline{\bar{P}}_{2}$ and $\mathrm{D} \equiv \overline{\mathrm{P}}_{2} \mathrm{~A}$. Here $\overline{\mathrm{P}}_{2}$ is a $\mathrm{B}$-chain complex, hence by induction $\mathrm{D}^{\prime} \equiv \overline{\mathrm{P}}_{2}^{\bar{\prime}} \mathrm{A}^{\prime}$, in which $P_{2}$ and $P_{2}^{\prime}$ are similar. The remainder follows easily. (4a) Assume that $Q \bar{P}_{A} \geq_{\gamma}^{\prime} K$ is $Q Q_{1}[y, C] D \geq_{\gamma}^{\prime} Q Q_{1}\left[y, C^{\prime}\right] D^{\prime}$ as a direct consequence of $Q Q_{1} C \geq_{Y}^{\prime} Q Q_{1} C^{\prime}$ and $Q Q_{1}[y, C] D \geq_{Y}^{\prime} Q Q_{1}[y, C] D^{\prime}$. Then $\overline{\mathrm{P}} \equiv Q_{1}[y, C] \overline{P_{2}}$ and $D \equiv \overline{P_{2}}$. The completion of the proof is similar to that in the last part of the previous case. (4b) Assume that $Q \overline{\bar{P} A} z_{\gamma}^{\prime} K$ is $Q_{0}[y, C] Q_{1} \overline{\bar{P} A} z_{\gamma}^{\prime} Q_{0}\left[y, C^{\prime}\right] D^{\prime}$ as a direct consequence of $Q_{0} C^{\prime} \geq_{Y}^{\prime} Q_{0} C^{\prime}$ and $Q_{0}[y, C] Q_{1} \bar{F}_{A} \geq_{Y}^{\prime} Q_{0}[y, C] D^{\prime}$. Then, by induction, $D^{\prime} \equiv Q_{1}^{\prime} \bar{P}^{\prime} A^{\prime}$ with $\left\|Q_{1}\right\|=\left\|Q_{1}^{\prime}\right\|$, while $\overline{\overline{\mathrm{P}}}$ and $\overline{\overline{\mathrm{P}}}$ ' are similar. The remainder follows.

(5) Assume that $Q \overline{\overline{\mathrm{P}}} \overline{\geq^{\prime}} \geq_{\gamma}^{\prime} K$ is a direct consequence of $\overline{Q \bar{P}} \geq_{Y}^{\prime} K^{\prime}$ and $K^{\prime} \geq_{\alpha} K$. The proof is again by induction.

Corollary 6.31. If $Q \hat{P A} \in \Delta$ and $Q \hat{P A} \geq_{\gamma}^{\prime} K$, then $K \equiv Q^{\prime} \hat{P}^{\prime} A^{\prime}$, where $\|Q\|=\left\|Q^{\prime}\right\|, Q \geq_{\gamma}^{\prime} Q^{\prime}, Q \hat{P A} z_{\gamma}^{\prime} Q P^{\prime} A^{\prime}$, while $P$ and $P^{\prime}$ are similar. 
The following four theorems are lemmas for Th. 6.36. Th. 6.34 might have been called "the substitution lemma for y-reduction".

\section{Theorem 6.32 .}

(1) Let $Q \hat{P}[x, B] C \in \Delta$ and $Q \hat{P}[x, B] C \geq_{\gamma}^{\prime} K$. Then $K \equiv Q^{\prime} \hat{P^{\prime}}\left[x, B^{\prime}\right] C^{\prime}$ such that $Q \geq \geq_{\gamma}^{\prime} Q^{\prime}, Q \hat{P}[x, B] C \geq P_{\gamma}^{\prime} Q P^{\prime}\left[x, B^{\prime}\right] C^{\prime},\|Q\|=\left\|Q^{\prime}\right\|$, while $P$ and $P^{\prime}$ are similar.

(2) Let $Q\{A\} \hat{P}[x, B] C \in \Delta$ and $Q\{A\} \hat{P}[x, B] C \geq_{Y}^{\prime} K$. Then either (i) $K \equiv Q^{\prime}\left\{A^{\prime}\right\} P^{\gamma}\left[x, B^{\prime}\right] C^{\prime}$, or (ii) $K \equiv Q^{\prime}\left\{A^{\prime}\right\} P^{\prime}\left[x, B^{\prime}\right]\left(x:=A^{\prime}\right) C^{\prime}$, where in both cases $Q \geq_{\gamma}^{\prime} Q^{\prime}, Q \geq_{Y}^{\prime} Q A^{\prime}, Q \hat{P}[x, B] C \geq_{Y}^{\prime} Q P^{\prime}\left[x, B^{\prime}\right] C^{\prime}$ and $\|Q\|=\left\|Q^{\prime}\right\|$, while $P$ and $P^{\prime}$ are similar.

(3) Let $Q\{A\} B \in \Delta$ and $Q\{A\} B \geq \geq_{\gamma}^{\prime} K$. Then either (i) $K \equiv Q^{\prime}\left\{A^{\prime}\right\} B^{\prime}$ where $Q \geq \geq_{\gamma}^{\prime} Q^{\prime}, Q A \geq \geq_{\gamma}^{\prime} Q A^{\prime}, Q B \geq \geq_{\gamma}^{\prime} Q B^{\prime}$ and $\|Q\|=\left\|Q^{\prime}\right\|$, or (ii) $Q\{A\} B \equiv Q\{A\} \hat{P}[x, C] D, x \subset D, K \equiv Q^{\prime}\left\{A^{\prime}\right\} \hat{P^{\prime}}\left[x, C^{\prime}\right]\left(x:=A^{\prime}\right) D^{\prime}, Q \geq_{Y}^{\prime} Q^{\prime}$, $\|Q\|=\left\|Q^{\prime}\right\|, Q A \geq_{\gamma}^{\prime} Q A^{\prime}$ and $Q \hat{P}[x, C] D \geq_{\gamma}^{\prime} Q P^{\prime}\left[x, C^{\prime}\right] D^{\prime}$.

Proof. See Cor.6.31 and the possibilities for single-step $\gamma$-reduction.

Theorem 6.33. If $\hat{Q P}[\mathrm{x}, \mathrm{B}] \mathrm{C} \in \Delta, \hat{\mathrm{Q}}[\mathrm{x}, \mathrm{B}] \mathrm{C} \geq_{\gamma}^{\prime} \mathrm{Q}^{\prime} \hat{\mathrm{P}}^{\prime}\left[\mathrm{x}, \mathrm{B}^{\prime}\right] \mathrm{C}^{\prime}$ and $\mathrm{x} \subset \mathrm{C}$, then $x \subset C^{\prime}$.

Proof. In a subexpression we can only eliminate free variables by substitution, and substitution can only originate from a $\beta_{1}$-reduction. Note that a $B_{1}$-reduction yielding a substitution $(x:=A)$ cannot occur in the above.

Theorem 6.34. Let $\mathrm{QA}$ and $\mathrm{Q} \overline{\overline{\mathrm{P}}} \mathrm{B} \in \Delta$, let no binding variable of $\overline{\overline{\mathrm{P}}} \mathrm{B}$ occur in $Q A$ and let $x$ not occur in $\overline{\overline{\mathrm{P}}}$. Let $Q \overline{\overline{\mathrm{P}}} \mathrm{B} \geq_{y}^{\prime} \mathrm{Q}^{\overline{\mathrm{P}^{\prime}}} \mathrm{B}^{\prime}$ (where $\left.\|P\|=\left\|P^{\prime}\right\|\right)$ and $Q A \geq_{\gamma}^{\prime} Q A^{\prime}$. Then $Q \overline{\bar{P}}(x:=A) B \geq_{Y}^{\prime} Q P^{\prime \prime}\left(x:=A^{\prime}\right) B^{\prime}$.

Proof. First consider the case that $P \equiv Q_{1}$, so $P^{\prime} \equiv Q_{1}^{\prime}$. We prove the theorem by induction on $|B|$. If $B \equiv y \neq x$ or $B \equiv \tau$ then the theorem is trivial. If $B \equiv x$, note that $Q Q_{1} A \geq_{\gamma}^{\prime} Q Q_{1} A^{\prime}$, so also $Q_{1} A \geq_{\gamma}^{\prime} Q_{1}^{\prime} A^{\prime}$ by induction on the length of proof of $Q Q_{1} x \geq_{\gamma}^{\prime} Q Q_{1}^{\prime} x$ and monotony rule (3) for single-step $\gamma$-reduction. 
(1) Let $B \equiv[y, E] F$. Then $B^{\prime} \equiv\left[y, E^{\prime}\right] F^{\prime}$ by Th. 6.32(1), $Q Q_{1} E \geq Q_{\gamma}^{\prime} Q_{1} E^{\prime}$ and $Q Q_{1}[y, E] F \geq Q_{Y}^{\prime} Q_{1}[y, E] F^{\prime}$. So also (by induction) $Q Q_{1}(x:=A) E \geq_{Y}^{\prime} Q Q_{1}\left(x:=A^{\prime}\right) E^{\prime}$ and $Q Q_{1}[y, E](x:=A) F \geq_{Y}^{\prime} Q Q_{1}[y, E]\left(x:=A^{\prime}\right) F^{\prime}$. It easily follows from the latter reduction that $Q Q_{1}[y,(x:=A) E](x:=A) F \geq_{Y}^{\prime} Q Q_{1}[y,(x:=A) E]\left(x:=A^{\prime}\right) F^{\prime}$. It follows that $Q Q_{1}(x:=A) B \geq_{\gamma}^{\prime} Q Q_{1}\left(x:=A^{\prime}\right) B^{\prime}$, hence also $Q Q_{1}(x:=A) B \geq_{\gamma}^{\prime} Q Q_{1}^{\prime}\left(x:=A^{\prime}\right) B^{\prime}$.

(2) Let $B \equiv\{E\} F$. Now by Th. 6.32(3) either $B^{\prime} \equiv\left\{E^{\prime}\right\} F^{\prime}$ with $Q Q_{1} E \geq_{\gamma}^{\prime} Q Q_{1}^{\prime} E^{\prime}$ and $Q Q_{1} F \geq_{\gamma}^{\prime} Q Q_{1}^{\prime} F^{\prime}$, or $B \equiv\{E\} \hat{P}_{1}[y, G] H$, and $Q Q_{1} B \equiv Q Q_{1}\{E\} \hat{P}_{1}[y, G] H \geq_{\gamma}^{\prime} Q Q_{1}^{\prime}\left\{E^{\prime}\right\} \hat{P}_{1}^{\dagger}\left[y, G^{\prime}\right]\left(y:=E^{\prime}\right) H^{\prime} \equiv Q Q_{1}^{\prime} B^{\prime}$. In the first case the proof is similar to the proof in case (1). In the second case we can follow analogous lines, using the fact that $\left(y:=\left(x:=A^{\prime}\right) E^{\prime}\right)\left(x:=A^{\prime}\right) H^{\prime} \equiv\left(x:=A^{\prime}\right)\left(y:=E^{\prime}\right) H^{\prime}$ but for renaming .

Now consider the case that $P \not Q_{1}$, whence at least one applicator must occur in chain $P$. We proceed with induction on the length of proof of $\mathrm{Q}^{\overline{\mathrm{P}}} \overline{\mathrm{B}^{\prime}} \geq_{\gamma}^{\prime} \mathrm{QP}^{\prime} \mathrm{\overline {P }}^{\prime}$.

(1) Assume that $\mathrm{Q}^{\overline{\bar{P}} \mathrm{~B}} \geq_{\gamma}^{\prime} \mathrm{QP}^{\prime} \mathrm{B}^{\prime}$ by reflexivity. This case can be proved similarly to the case that $\overline{\overline{\mathrm{P}}} \equiv Q_{1}$.

(2) Assume that $Q \overline{\mathrm{P}_{B}} \geq_{\gamma}^{\prime} Q \mathrm{P}^{\top} \mathrm{B}^{\prime}$ is $Q Q_{1}\{C\} \hat{\mathrm{P}}_{1}[y, D] E \geq_{Y}^{\prime}$

$Q Q_{1}\left\{C^{\prime}\right\} \hat{P_{i}}\left[y, D^{\prime}\right]\left(y:=C^{\prime}\right) E^{\prime}$ as a direct consequence of $Q Q_{1} C \geq_{\gamma}^{\prime} Q Q_{1} C^{\prime}$ and $Q Q_{1} \hat{P}_{1}[y, D] E \geq_{\gamma}^{\prime} Q Q_{1} \hat{P}_{1}^{\prime}\left[y, D^{\prime}\right] E^{\prime}$. Now it must hold that $E \equiv \bar{P}_{2} B^{B}$ and $E^{\prime} \equiv \bar{P}_{2}^{\overline{7}} B^{\prime}$, where $B^{\prime} \equiv\left(y:=C^{\prime}\right) B^{\prime \prime}$. Since $Q_{1} \hat{P}_{1}[y, D] P_{2}$ is also a Brchain complex, it follows by induction: $Q_{1} \hat{P}_{1}[y, D] P_{2}^{\prime \prime}(x:=A) B \geq_{\gamma}^{\prime} Q_{1} \hat{P}_{1}\left[y, D^{\prime}\right] P_{2}^{\prime \prime}\left(x:=A^{\prime}\right) B^{\prime \prime}$, so $Q \overline{\bar{P}}(x:=A) B \equiv$ $Q Q_{1}\{C\} \hat{P}_{1}[y, D] P_{2}^{=}(x:=A) B \geq_{y}^{\prime} Q Q_{1}\left\{C^{\prime}\right\} \hat{P}_{1}^{\prime}\left[y, D^{\prime}\right]\left(y:=C^{\prime}\right)\left(P_{2}^{\top}\left(x:=A^{\prime}\right) B^{\prime \prime}\right) \equiv$ $Q Q_{1}\left\{C^{\prime}\right\} \hat{P}_{1}^{\prime}\left[y, D^{\prime}\right]\left(\left(y:=C^{\prime}\right) P_{2}^{\bar{\gamma}}\right)\left(x:=A^{\prime}\right)\left(y:=C^{\prime}\right) B^{\prime \prime} \equiv Q P^{\prime}\left(x:=A^{\prime}\right) B^{\prime}$ (here we changed $\left(y:=C^{\prime}\right)\left(x:=A^{\prime}\right) B^{\prime \prime}$ into $\left(x:=A^{\prime}\right)\left(y:=C^{\prime}\right) B^{\prime \prime}$, which is allowed by Th. 6.33 and by the conditions imposed upon the variables). (3) Assume that $Q_{\bar{P} B} \geq_{\gamma}^{\prime} Q \bar{P}^{\prime} B^{\prime}$ is $Q Q_{1}\{C\} D Z_{\gamma}^{\prime} Q Q_{1}\left\{C^{\prime}\right\} D^{\prime}$ as a direct consequence of $Q Q_{1} C \geq_{\gamma}^{\prime} Q Q_{1} C^{\prime}$ and $Q Q_{1} D_{Y}^{\prime} Q Q_{1} D^{\prime}$. Then $\overline{\bar{P}} \equiv Q_{1}\{C\} \bar{P}_{2}$ and $\overline{P^{\prime}} \equiv Q_{1}\left\{C^{\prime}\right\} P_{2}^{\bar{F}} ; D \equiv P_{2}=1$ and $D^{\prime} \equiv P_{2} B^{\prime}$. By induction: $Q Q_{1} \bar{P}_{2}(x:=A) B \geq_{\gamma}^{\prime} Q Q_{1} \overline{\bar{P}}\left(x:=A^{\prime}\right) B^{\prime}$, so al so $Q \overline{\bar{P}}(x:=A) B z_{\gamma}^{\prime} Q \overline{P^{\prime}}\left(x:=A^{\prime}\right) B^{\prime}$. (4) Assume that $Q^{\bar{P}} \geq_{\gamma}^{\prime} Q P^{\prime} B^{\prime}$ is $Q Q_{1}[y, C] D \geq_{\gamma}^{\prime} Q Q_{1}\left[y, C^{\prime}\right] D^{\prime}$ as a direct consequence of $Q Q_{1} C \geq_{Y}^{\prime} Q Q_{1} C^{\prime}$ and $Q Q_{1}[y, C] D \geq_{Y}^{\prime} Q Q_{1}[y, C] D^{\prime}$. 
Then $\overline{\mathrm{P}} \equiv Q_{1}[y, C] \overline{\mathrm{P}}_{2}^{ \pm}$and $\overline{\overline{\mathrm{P}^{\prime}}} \equiv Q_{1}\left[y, C^{\prime}\right] \overline{\mathrm{P}_{2}^{\prime}} ; \mathrm{D} \equiv \overline{\mathrm{P}_{2}} \mathrm{~B}$ and $D^{\prime} \equiv \overline{\overline{\mathrm{P}_{2}^{\prime}}} \mathrm{B}^{\prime}$. The remainder of the proof is analogous to that in case (3).

(5) Assume that $Q \overline{\bar{P}} B \geq_{\gamma}^{\prime} Q \bar{P}^{\prime} B^{\prime}$ is a direct consequence of $Q \overline{\bar{P} B} \geq_{\gamma}^{\prime} Q^{\prime \prime} \bar{P}^{\bar{\prime}} B^{\prime \prime}$ and $Q^{\prime \prime} P^{\overline{7}} B^{\prime \prime} \geq_{\alpha} Q \bar{P}^{\prime} B^{\prime}$. Then $Q \overline{\bar{P}}(x:=A) B \geq_{\gamma}^{\prime} Q^{\prime \prime} \bar{P}^{\bar{\prime}}\left(x:=A^{\prime}\right) B^{\prime \prime}$ by induction; the remainder is easy.

Theorem 6.35. If $Q A$ and $Q^{\prime} A^{\prime} \in \Delta_{i}^{\prime} Q \geq_{\gamma}^{\prime} Q^{\prime}$ and $Q A z_{\gamma}^{\prime} Q A^{\prime}$, then $Q A \geq Q_{Y}^{\prime} A^{\prime}$.

Proof. Induction on $\|Q\|$, using monotony rule (3) of single-step r-reduction.

Theorem 6.36. If $K \in \Delta, K \geq_{Y}^{\prime} L$ and $K \geq_{Y}^{\prime} M$, there is an $N$ such that $L \geq_{\gamma}^{\prime} N$ and $M \geq_{\gamma}^{\prime} N$.

Proof. Induction on the length of proof of $K \geq \frac{1}{Y}$.

We shall use Th. 6.24 and Th. 6.35 several times without saying so.

(1) Let $K \geq_{\gamma}^{\prime} L$ by reflexivity. Take $N \equiv M$.

(2) Let $K \geq_{Y}^{\prime} L$ be $Q\{A\} \hat{P}[x, B] C \geq_{Y}^{\prime} Q\left\{A^{\prime}\right\} \hat{P}^{\prime}\left[x, B^{\prime}\right]\left(x:=A^{\prime}\right) C^{\prime}$ as a direct consequence of $Q A \geq \geq_{\gamma}^{\prime} Q A^{\prime}$ and $Q \hat{P}[x, B] C \geq_{\gamma}^{\prime} Q \hat{P}^{\prime}\left[x, B^{\prime}\right] C^{\prime}$. Now by Th. 6.32 (2) $M$ can have the form (i) $M \equiv Q^{\prime \prime}\left\{A^{\prime \prime}\right\} \hat{P}^{\prime \prime}\left[x, B^{\prime \prime}\right] C^{\prime \prime}$ or (ii) $M \equiv Q "\left\{A^{\prime \prime}\right\} \hat{P}^{\prime \prime}\left[x, B^{\prime \prime}\right]\left(x:=A^{\prime \prime}\right) C^{\prime \prime}$, where in both cases $Q \geq_{\gamma}^{\prime} Q^{\prime \prime}$, $Q A \geq_{\gamma}^{\prime} Q A^{\prime \prime}, Q \hat{P}[x, B] C \geq_{\gamma}^{\prime} Q \hat{P}^{\prime \prime}\left[x, B^{\prime \prime}\right] C^{\prime \prime},\|Q\|=\left\|Q^{\prime \prime}\right\|$ and $\|P\|=\left\|P^{\prime \prime}\right\|$. By induction and by Th. 6.22 there is an $A^{\prime \prime \prime}$ such that $Q A^{\prime} \geq \geq_{\gamma}^{\prime} Q^{\prime \prime \prime}$ and $Q A^{\prime \prime} z_{\gamma}^{\prime} Q A^{\prime \prime \prime}$. Again by induction and by Th. 6.32(1) there are $\hat{P}^{\prime \prime \prime}, B^{\prime \prime \prime}$ and $C^{\prime \prime \prime}$ such that $\hat{P}^{\prime}\left[x, B^{\prime}\right] C^{\prime} z_{Y}^{\prime} \hat{Q}^{\prime \prime \prime}\left[x, B^{\prime \prime}\right] C^{\prime \prime \prime}$ and $Q \hat{P}^{\prime \prime}\left[x, B^{\prime \prime}\right] C^{\prime \prime} \geq_{\gamma}^{\prime} Q^{\prime \prime \prime}\left[x, B^{\prime \prime \prime}\right] C^{\prime \prime \prime}$. By Th. 6.34:

$Q \hat{P}^{\prime}\left[x, B^{\prime}\right]\left(x:=A^{\prime}\right) C^{\prime} \geq_{\gamma}^{\prime} Q \hat{P}^{\prime \prime '}\left[x, B^{\prime \prime \prime}\right]\left(x:=A^{\prime \prime \prime}\right) C^{\prime \prime \prime}$, hence by monotony

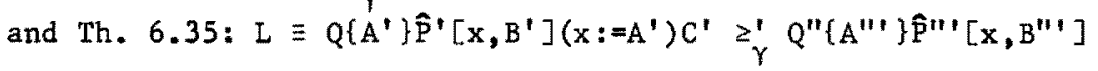
$\left(x:=A^{\prime \prime \prime}\right) C^{\prime \prime \prime}$. Call the latter expression $N$.

It al so holds that $Q " \hat{P} "\left[x, B^{\prime \prime}\right] C^{\prime \prime} \geq_{\gamma}^{\prime} Q " \hat{P} " '\left[x, B^{\prime \prime}\right] C^{\prime \prime \prime}$, and $x \in C$ '" by Th. 6.33. So $Q^{\prime \prime}\left\{A^{\prime \prime}\right\} \hat{P}^{\prime \prime}\left[x, B^{\prime \prime}\right] C^{\prime \prime} \geq_{\gamma}^{\prime} N \equiv Q^{\prime \prime}\left(A^{\prime \prime \prime}\right\} \hat{P}^{\prime \prime \prime}\left[x, B^{\prime \prime \prime}\right]\left(x:=A^{\prime \prime \prime}\right) C^{\prime \prime \prime}$ by an elementary $\gamma$-reduction. This completes this part of the proof in case (i). In case (ii) we first establish that $Q P^{\prime \prime}\left[x, B^{\prime \prime}\right]\left(x:=A^{\prime \prime}\right) C^{\prime \prime} \geq_{\gamma}^{\prime} Q \hat{P} " \prime\left[x, B^{\prime \prime \prime}\right]\left(x:=A^{\prime \prime \prime}\right) C$ "' by Th. 6.34, yielding by monotony that $M z_{\gamma}^{\prime} N$. 
(3) Let $K \geq_{\gamma}^{\prime} L$ be $Q\{A\} C \geq_{\gamma}^{\prime} Q\left\{A^{\prime}\right\} C^{\prime}$ as a direct consequence of $Q A \geq_{\gamma}^{\prime} Q A^{\prime}$ and $Q C \geq_{\gamma}^{\prime} Q C^{\prime}$. Now by Th. 6.32(3) $M$ can have the form (i) $M \equiv Q "\left\{A^{\prime \prime}\right\} C^{\prime \prime}$, with $Q \geq Q^{\prime \prime},\|Q\|=\|Q "\|, Q A \geq \geq_{\gamma}^{\prime} Q A^{\prime \prime}$ and $Q C \geq_{Y}^{\prime} Q C^{\prime \prime}$, or (ii) $M \equiv Q "\left\{A^{\prime \prime}\right\} \hat{P}^{\prime \prime}\left[x, D^{\prime \prime}\right]\left(x:=A^{\prime \prime}\right) E^{\prime \prime}$, where $K \equiv Q\{A\} \hat{P}[x, D] E, x \subset E$, $Q A \geq_{\gamma}^{\prime} Q A^{\prime}, Q \geq_{Y}^{\prime} Q^{\prime \prime}$ and $Q \hat{P}[x, D] E \geq_{Y}^{\prime} Q \hat{P}^{\prime \prime}[x, D "] E^{\prime \prime}$.

In case (i) we can find by induction $A^{\prime \prime \prime}$ and $C^{\prime \prime \prime}$ such that

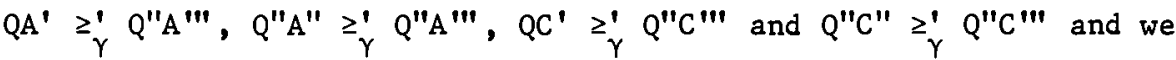
can take $N \equiv Q^{\prime \prime}\left\{A^{\prime \prime \prime}\right\} C^{\prime \prime \prime}$. In case (ii) we are in a position similar to case (i) of (2), with $L$ and M permuted.

(4) Let $K \geq_{\gamma}^{\prime}$ L be $Q[x, A] C \geq_{\gamma}^{\prime} Q\left[x, A^{\prime}\right] C^{\prime}$ as a direct consequence of $\mathrm{QA} \geq_{\gamma}^{\prime} \mathrm{QA} \mathrm{A}^{\prime}$ and $\mathrm{Q}[\mathrm{x}, \mathrm{A}] \mathrm{C} \geq_{\gamma}^{\prime} \mathrm{Q}[\mathrm{x}, \mathrm{A}] \mathrm{C}^{\prime}$. Then $\mathrm{M} \geq_{\alpha} \mathrm{Q}^{\prime \prime}\left[\mathrm{x}, \mathrm{A}^{\prime \prime}\right] \mathrm{C}^{\prime \prime}$ by Th. 6.32(1), where $Q \geq_{\gamma}^{\prime} Q^{\prime \prime}, Q A \geq_{\gamma}^{\prime} Q A^{\prime \prime}$ and $Q[x, A] C \geq_{\gamma}^{\prime} Q[x, A] C^{\prime \prime}$. Take $\mathrm{N} \equiv Q^{\prime \prime}\left[\mathrm{x}, \mathrm{A}^{\prime \prime \prime}\right] \mathrm{C}^{\prime \prime \prime}$, where $\mathrm{A}^{\prime \prime \prime}$ and $C^{\prime \prime \prime}$ are obtained as in (3), case (i).

(5) Let $\mathrm{K} \geq_{\gamma}^{\prime} \mathrm{L}$ as a direct consequence of $\mathrm{K} z_{\gamma}^{\prime} \mathrm{L}^{\prime}$ and $\mathrm{L}^{\prime} z_{\alpha} \mathrm{L}$. Then by induction we can find an $N$ such that $L^{\prime} \geq_{\gamma}^{\prime} N$ and $M \geq_{\gamma}^{\prime} N$, and also $L \geq \geq_{Y}^{\prime}$.

Theorem 6.37 (CR for $\gamma$-reduction). If $\mathrm{K} \in \Delta, \mathrm{K} \geq_{\gamma} \mathrm{L}$ and $\mathrm{K} \geq_{\gamma} \mathrm{M}$, then $\mathrm{L} \sim \sim_{\gamma}$.

Proof. This is a consequence of the previous theorem.

Theorem 6.38 (CR for $\beta_{1}$-reduction). If $K \in \Delta, K \geq_{\beta_{1}} L$ and $K \geq_{\beta_{1}}{ }^{M}$, then $L \sim_{\beta_{1}}$ M but for $\alpha$-reduction.

Proof. Decompose $K \geq_{\beta_{1}} L$ and $K \geq_{\beta_{1}} M$, apply Th. 6.25, Th. 6.37 and Th. 6.26: we obtain an $N^{\prime}$ such that $L \geq_{\beta_{1}} N_{\alpha} \geq^{\prime}$ and $M \geq_{\beta_{1}} N^{\prime \prime} \geq_{\alpha} N^{\prime}$.

Theorem 6.39. If $K \in \Delta, K \geq_{\beta_{1}}^{\prime} L$ and $K \geq_{B_{2}}^{\prime} M$, then there is an $n$ such that $M \geq \geq_{\beta_{1}}^{\prime} N$ and $L \geq_{\beta_{2}}^{\mathfrak{n}} N$ with $n \geq 1$.

Proof. Let $K \geq_{\beta_{1}^{\prime}}^{\prime}$ L be generated by $Q\{A\} \hat{P}[x, B] C \geq_{\beta_{1}^{\prime}}^{\prime} Q\{A\} \hat{P}[x, B](x:=A) C$, and $K \geq_{\beta_{2}}^{\prime} M$ by $Q^{\prime}\{D\} \hat{\mathrm{P}}_{1}[\dot{y}, E] F \geq_{\beta_{2}}^{\prime} Q^{\prime} \hat{\mathrm{P}}_{1} F$. If $\{D\} \hat{\mathrm{P}}_{1}[y, E] F \subset A$, we need $n$ 
$B_{2}$-reductions for the $n A^{\prime} s$ in $Q\{A\} \hat{P}\left[x^{\circ}, B\right](x:=A) C$. If not, we need only one. The theorem easily follows.

Theorem 6.40. If $\mathrm{K} \in \Delta, \mathrm{K} \geq_{\beta_{1}} \mathrm{~L}$ and $\mathrm{K} \geq_{B_{2}} \mathrm{M}$, there is an $\mathrm{N}$ such that $M \geq_{B_{1}} N$ and $L \geq_{B_{2}} N$.

Proof. Apply Th. 6.39 repeatedly. This can be illustrated by the following diagram

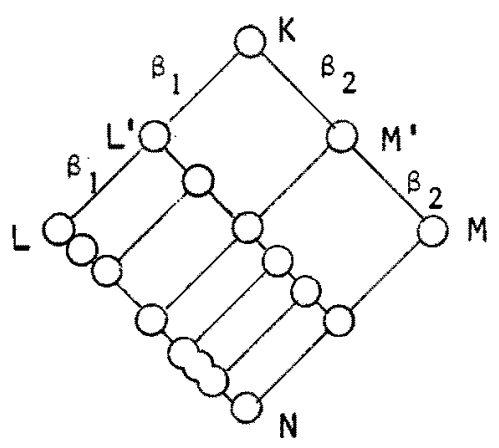

Here we assume that $K \geq_{B_{1}} L$ can be decomposed into $K \geq_{B_{1}}^{\prime} L^{\prime} \geq_{B}^{\prime} I_{1}$, and $K \geq_{B_{2}} M$ into $K \geq_{B_{2}}^{\prime} M^{\prime} \geq_{B_{2}}^{\prime} M$. In the diagram all edges (in the sense usual in graph theory) parallel to the edge from $K$ to $L$ ' represent single-step $\beta_{1}$-reductions, those in the direction of $\mathrm{K} \geq_{\beta_{2}}^{\prime} M^{*}$ represent single-step $B_{2}$-reductions.

Theorem 6.41. If $K \in \Delta, K \geq_{B_{2}}^{\prime} L$ and $K \geq_{B_{2}}^{\prime} M$, there is an $N$ such that $\mathrm{L} \geq_{\mathrm{B}_{2}}^{\prime} \mathrm{N}$ or $\mathrm{L} \equiv \mathrm{N}$, and $\mathrm{M} \geq_{\mathrm{B}_{2}}^{\prime} \mathrm{N}$ or $\mathrm{M} \equiv \mathrm{N}$.

Proof. Let $\mathrm{K} \geq_{\mathrm{B}_{2}^{\prime}}^{\prime} \mathrm{L}$ be generated by $\mathrm{Q}\{\mathrm{A}\} \hat{\mathrm{P}}[\hat{\mathrm{X}}, \mathrm{B}] \mathrm{C} \geq_{\mathrm{B}_{2}}^{\prime} \hat{\mathrm{QPC}}$, and $\mathrm{K} \geq_{\mathrm{B}_{2}}^{\prime} \mathrm{M}$ by $Q^{\prime}\{D\} \hat{P}_{1}\left[\dot{y}_{,} E\right] \mathrm{F}_{B_{2}}^{\prime} Q^{\prime} \hat{\mathrm{P}}_{1} F$. If $(D\} \hat{\mathrm{P}}_{1}[\dot{y}, E] F \subset A$ or $\subset B$ then $M_{B_{2}}^{\prime} L$; if $\{A\} \hat{P}[\stackrel{\circ}{x}, B] C \subset D$ or $\subset E$, then $L z_{B_{2}}^{:} M$. If $\{D\} \hat{\mathrm{P}}_{1}[\dot{y}, E] F \equiv\{A\} P[\hat{x}, B] C$ then $L \equiv M$. In all other cases there is clearly an $N$ such that $L \geq_{B_{2}}^{\prime} N$ and $M \geq_{B_{2}}^{\prime} N$. 
Theorem 6.42 (CR for $B_{2}$-reduction). If $K \in \Delta, K \geq_{B_{2}} L$ and $K \geq_{B_{2}} M$, then $\mathrm{L} \sim_{\mathrm{B}_{2}} \mathrm{M}$.

Proof. Apply Th. 6.41 repeatedly.

Theorem 6.43 (CR for B-reduction). If $K \in \Delta, K \geq_{\beta} L$ and $K \geq_{\beta} M$, then $L \sim_{\beta} M$.

Proof. Decompose $K \geq_{B} L$ and $K \geq_{B} M$, according to Th. 6.19, into $K \geq{ }_{B_{1}} L^{\prime} \geq_{B_{2}} L$ and $K \geq_{B_{1}} M^{\prime} \geq_{B_{2}} M$ respectively. The remainder of the proof is illustrated by the following diagram:

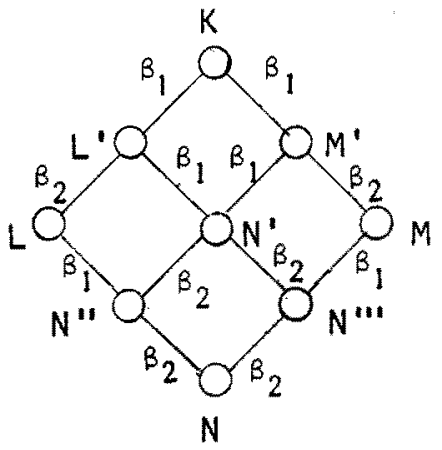

We find $N^{\prime}$ from Th. $6.38, N^{\prime \prime}$ and $N^{\prime \prime \prime}$ from $T h .6 .40$ and finally $N$ from Th. 6.42 .

\section{\$ 7. n-REDUCTION, REDUCTION AND LAMBDA-EQUTVALENCE}

A third reduction in lambda-calculus (apart from $\alpha$ - and $\beta-$ reduction) is called n-reduction and denoted by $\geq_{n}$. We shall incorporate it in our system.

We first define single-step n-reduction, denoted by $\geq_{n}^{*}$

Definition 7.1. Single-step n-reduction is the relation generated by:

(1) If $Q[x, A]\{x] B \in \Delta$ and $x \neq B$, then $Q[x, A](x) B \geq \geq_{\eta}^{\prime} Q B$. 
(2) Let $Q\{A\} C$ and $Q\{A\} D \in \Delta$. If $Q C \geq_{n}^{\prime} Q D$, then $Q\{A\} C \geq_{n}^{\prime} Q\{A\} D$.

(3) Let $Q[x, A] C$ and $Q[x, B] C \in \Delta$. If $Q A \geq_{\eta}^{\prime} Q B$, then $Q[x, A] C \geq_{\eta}^{\prime}$ $Q[x, B] C$.

(4) Let $Q\{A\} C$ and $Q\{B\} C \in \Delta$. If $Q A \geq_{\eta}^{\prime} Q B$, then $Q\{A\} C \geq_{\eta}^{\prime} Q\{B\} C$.

Rules (2), (3) and (4) are called the monotony rutes of singlestep $n$-reduction; they are similar to those of single-step $B$-reduction. Rule (l) is called the rule of elementary n-reduction.

Definition 7.2. n-reduction is the reflexive and transitive closure of single-step n-reduction.

If $A$ and $B$ are related by a (single-step) n-reduction, we speak of "the (single-step) n-reduction $A z_{n}^{\prime} B$ ". The notions n-step nreduction and decomposition of an n-reduction are defined analogously to the corresponding notions for B-reduction. If the first derivation step of a single-step n-reduction has the form $Q[x, A]\{x\} B \geq_{n}^{\prime} Q B$, we say that $Q[x, A]\{x\} B$ generates the single-step n-reduction.

Theorem 7.3. Let $K \in \Delta$. Then $Q[x, A]\{x\} B$ generates a single-step $n-$ reduction of the form $K \geq_{\eta}^{\prime} L$ if and only if $[x, A]\{x\} B \subset K$ and $\mathrm{Q}[\mathrm{x}, \mathrm{A}]\{\mathrm{x}\} \mathrm{B} \equiv \mathrm{K} \mid[\mathrm{x}, \mathrm{A}]\{\mathrm{x}\} \mathrm{B}$.

Proof. Similar to the proof of Th. 5.7.

Theorem 7.4. If $K \in \Delta$ and $K \geq_{\eta}^{\prime} L$, then $L \in \Delta$.

Proof. Induction on the length of proof of $\mathrm{K} \geq_{\eta}^{\prime} L$. The proof is similar to the proof of Th. 5.8.

Theorem 7.5. If $Q E \in \Delta, Q E \geq_{n}^{\prime} Q^{\prime} F$ and $\|Q\|=\left\|Q^{\prime}\right\|$, then (i) $Q \equiv Q^{\prime}$, (ii) $E \equiv F$, or (iii) $Q \equiv Q_{0}[x, A], E \equiv\{x\}[y, B] E^{\prime}, Q^{\prime} \equiv Q_{0}[y, B]$, $x \notin[y, B] E^{\prime}$ and $F \equiv E^{\prime}$. In the second case $Q \equiv Q_{1}[x, K] Q_{2}$, $Q^{\prime} \equiv Q_{1}[X, L] Q_{2}$ and $Q_{1} K \geq_{n}^{\prime} Q_{1} L$.

Proof. Induction on the length of proof of $Q E \geq_{n}^{\prime} Q^{\prime} F$. The proof is comparable to the proof of Th. 5.11, except for the case in which 
$Q E \geq_{n}^{\prime} Q^{\prime} F$ is an elementary n-reduction. In this case we have to note the possibility that $Q E$ and $Q^{\prime} F$ are as in (iii).

Theorem 7.6. If $Q E \in \Delta$ and $Q E \geq_{n}^{\prime} K$, then $K \equiv Q^{\prime} F^{\prime}$ for certain $Q^{\prime}$ and $F^{\prime}$ with $\left\|Q^{\prime}\right\| \geq\|Q\|-1$.

Proof. Similar to the proof of Th. 5.12.

Theorem 7.7. If $Q E \in \Delta$ and $Q E \geq \frac{1}{n} \mathrm{~K} \geq{ }_{\eta} Q G$, then $K \equiv Q F$.

Proof. If $Q \equiv Q_{1}[x, A]$ and $Q E \geq \geq_{n}^{\prime} K$ is $Q_{1}[x, A]\{x\} B \geq_{n}^{\prime} Q_{1} B$, then the binding variable $x$ of $K$ has disappeared, and we cannot regain it by $n$-reduction. Hence by $T h .7 .6: K \equiv Q^{\prime} F$ and $\left\|Q^{\prime}\right\|=\|Q\|$, and the case expressed in Th. 7.5 (iii) does not hold. In the derivation steps leading to $K \geq_{n} Q G$ the final ones of the first \|Q\|l abstractors cannot disappear by an elementary n-reduction for the same reason as above. Assume that $Q \neq Q^{\prime}$. Then by Th. 7.5 (ii): $Q \equiv Q_{1}[x, K] Q_{2}$, $Q^{\prime} \equiv Q_{1}[x, L] Q_{2}$ and $Q_{1} K \geq_{\eta}^{\prime} Q_{1} L$. It is clear that $|L|<|K|$. Since the length of an expression cannot increase by $n$-reduction it follows that we cannot regain $Q$ from $Q^{\prime}$. Hence $Q \equiv Q^{\prime}$.

Theorem 7.8. The monotony rules hold for n-reduction.

Proof. Similar to the proof of Th. 5.15, using Th. 7.5.

Theorem 7.9. If $\mathrm{QE}, \mathrm{PE}$ and $\mathrm{PF} \in \Delta$, and $\mathrm{QE} \geq_{\eta} \mathrm{QF}$, then $\mathrm{PE} \geq_{n} \mathrm{PF}$, Proof. Analogous to the proof of Th. 5.17; use Th. 7.7.

The converse of this theorem holds too.

Given an n-reduction $Q K \geq_{n} M$, it need not follow that $M \equiv Q^{\prime} N^{\prime}$ with $\|Q\|=\left\|Q^{\prime}\right\|$, since the final abstractors of $Q$ may have been cancelled in $n$-reductions. For example: Let $Q \equiv Q^{\prime}[x, A]$ and $K \equiv\{x\}_{\tau}$, then $Q K z_{n}^{\prime} Q^{\prime} \tau$, where $\left\|Q^{\prime}\right\|=\|Q\|-1$. This kind of $n-$ reductions plays an important rôle in the following. We shall call them $n$ :-reductions. We shall prove a number of theorems concerning $n$ :-reductions. In Th. 7.14 we shall show that we can postpone $n$ :reductions until after other n-reductions. Cor. 7.17 will result from our discussions of $n$ :-reductions. 
Definition 7.10 .

(1) $K \geq_{\eta}^{\prime} \mathrm{L}$ is called a single $\eta$ !-reduction (denoted by $K \geq_{\eta}^{(1)} \mathrm{L}$ ) if $K \equiv Q[x, A]\{x\} B$ and $K$ generates $K \geq_{n}^{\prime} L$ (i.e. if $K \geq_{n}^{\prime} L$ is an elementary $n$-reduction). This reduction is called of order $p$ if $\|\mathrm{Q}[\mathrm{x}, \mathrm{A}]\|=\mathrm{p}$.

(2) $K \geq_{\eta} L$ is called a k-fold $n$ !-reduction (denoted $k \geq_{n !}^{(k)} L$ ) if there are $K_{i} \equiv Q\left[x_{1}, A_{1}\right] \ldots\left[x_{i}, A_{i}\right]\left\{x_{i}\right\} \ldots\left\{x_{1}\right\} B$ such that $\mathrm{K} \equiv \mathrm{K}_{\mathrm{k}} \geq_{n !}^{(1)} \mathrm{K}_{\mathrm{k}-1} \ldots \geq_{n !}^{(1)} \mathrm{K}_{0} \equiv \mathrm{QB} \equiv \mathrm{L}$ and $\mathrm{K}_{\mathrm{i}}$ generates $\mathrm{k}_{i} \geq_{\eta}^{(1)} \mathrm{k}_{i-1}$. This reduction $\mathrm{k} \geq_{\eta}^{(k)} \mathrm{L}$ is called of order $\mathrm{p}$ if $\mathrm{K}_{\mathrm{k}} \geq \mathrm{n}_{\mathrm{n}}^{(1)} \mathrm{K}_{\mathrm{k}-1}$ is of order $\mathrm{p}$.

Theorem 7.11. If $k \geq . \Delta$ and $k z_{n !}^{(1)} I z_{n}^{\prime} N$, then either $k z_{n !}^{(2)} N$ or there is a reduction $K \geq_{n}^{1} M \geq_{n !}^{(1)} N$ where $K \geq_{n}^{\prime} M$ is not an $n !-$ reduction.

Proof. $K \equiv Q[x, A]\{x\} B \geq_{\eta !}^{(1)} Q B \equiv$ L. Consider the possibilities for $\mathrm{QB} \equiv L \geq \geq_{\eta}^{\prime} \mathrm{N}$.

Theorem 7.12. If $k \in \Delta$ and $K \geq_{\eta !}^{(k)} L \geq_{\eta}^{\prime} N$, where $K \geq_{\eta !}^{(k)} L$ is of order $p$, then either $\mathrm{K} \geq_{\eta !}^{(k+1)} N$ of $\operatorname{order} p$, or there is a reduction $K \geq_{\eta}^{\prime} M \geq_{\eta !}^{(k)} N$ where $M \geq_{n !}^{(k)} N$ is of order $p$ and $K \geq_{\eta}^{\prime} M$ is not an $n$ :reduction.

Proof. Compare with the previous theorem.

Theorem 7.13. Let $K \in \Delta$ and $K \geq_{n !}^{(k)} L \geq_{n} N$, where $K \geq_{n !}^{(k)} L$ is of order $p$. Then there is a reduction $K \geq_{n} L^{\prime} \geq_{n !}^{(l)} N$, where a decomposition of $K \geq_{\eta}$ L contains no $n !$-reductions and $L^{\prime} \geq_{\eta !}^{(l)} N$ is of order p.

Proof. Decompose $L \geq_{n} N$ into $L \equiv E_{1} \geq i \ldots \geq E_{n}^{\prime} \equiv N$.

We proceed with induction on $r$. If $r=1$ there is nothing to prove. Let $r>1$. Consider the reduction $K \geq_{n !}^{(k)} L \equiv E_{1} \geq_{n}^{\prime} E_{2}$. By the previous theorem we have either $K \geq_{n !}^{(k+1)} \mathrm{E}_{2}$ or a reduction $K \geq_{\eta}^{\prime} L^{\prime \prime} \geq_{\eta !}^{(k)} E_{2}$ where $K \geq_{n}^{\prime} L^{\prime \prime}$ is not an $n$ !-reduction. Applying the 
induction hypothesis on $\mathrm{K} \geq \frac{(k+1)}{n !} \mathrm{E}_{2} \geq_{n} \mathrm{~N}$ or $\mathrm{L}^{\prime \prime} \geq_{n !}^{(k)} \mathrm{E}_{2} \geq_{n} \mathrm{~N}$, we obtain $K \geq{ }_{n} L^{\prime} \geq_{n !}^{(l)} N$, where a decomposition of $K \geq_{\eta} L^{\prime}$ contains no $n$ :-reductions.

Theorem 7.14. If $Q K \in \Delta$ and $Q K \geq_{\eta} L$, there is a reduction $\mathrm{QK} \geq_{n} Q^{\prime} K^{\prime} \geq_{\eta}^{(k)}$ ! where $\|Q\|=\left\|Q^{\prime}\right\|, Q^{\prime} K \geq_{n !}^{(k)} L$ is of order $\|Q\|$, and where a decomposition of $Q K \geq{ }_{n} Q^{\prime} K^{\prime}$ contains no $n:-r e d u c t i o n s$ of order $\|Q\|$.

Proof. Decompose $\mathrm{QK} \geq_{n}$ L into single-step n-reductions $\mathrm{QK} \equiv \mathrm{L}_{1} \geq_{\eta}^{\prime} \ldots \geq_{n}^{\prime} \mathrm{L}_{\mathrm{n}} \equiv \mathrm{L}$. Let $i$ be the smallest integer such that $L_{i} \geq L_{i+1}^{\prime}$ is an $n !-r e d u c t i o n$ of order $\|Q\|$. Apply the previous theorem on $\mathrm{L}_{i} \geq \geq_{n}$ ! $\mathrm{L}_{i+1} \geq_{n} \mathrm{~L}_{\mathrm{n}} \equiv \mathrm{L}$. We obtain a reduction $\mathrm{QK} \geq_{n} L^{*} \geq_{n !}^{(k)} L$ as desired. The fact that $L^{\prime} \equiv Q^{\prime} K^{\prime}$ with $\left\|Q^{\prime}\right\|=\|Q\|$ follows from Th. 7.5.

Theorem 7.15. If $Q K \in \Delta, Q K \geq_{n} Q^{\prime} K^{\prime},\|Q\|=\left\|Q^{\prime}\right\|=p$ and a decomposition of $Q K \geq Q_{n}^{\prime} K^{\prime}$ contains no $n:$-reductions of order $P$, there is a reduction $Q K \geq_{n} Q^{\prime} \geq_{\eta} Q^{\prime} K^{\prime}$ and a reduction $Q K \geq_{n} Q^{\prime} K \geq_{n} Q^{\prime} K^{\prime}$. Proof. See Th. 7.5 .

Theorem 7.16. If $Q_{1} K \in \Delta, Q_{2} L \in \Delta, Q_{1} R \geq_{n} M, Q_{2} L \geq \geq_{n} M$, $Q_{1} \equiv\left[x_{1}, A_{1}\right] \ldots\left[x_{p}, A_{p}\right]$ and $Q_{2} \equiv\left[x_{1}, B_{1}\right] \ldots\left[x_{p}, B_{p}\right]$, there is an $N$ such that $Q_{1} K \geq{ }_{n} Q_{1} N$ and $Q_{2} L \geq Q_{2} N$.

Proof. By the aid of Th. 7.14 and Th. 7.15 we can find reductions

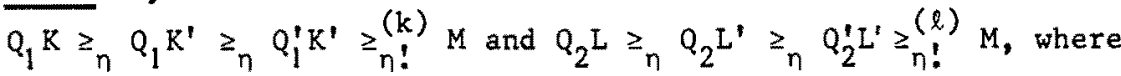
$Q_{!}^{\prime} K^{\prime} \geq_{\eta !}^{(K)} M$ and $Q_{2}^{\prime} L^{\prime} \geq_{n !}^{(t)} M$ are of order $p$. Note that $Q_{1}^{\prime} \equiv\left[x_{1}, A_{1}^{\prime}\right] \ldots\left[x_{p}, A_{p}^{\prime}\right]$ and $Q_{2}^{\prime} \equiv\left[x_{1}, B_{1}^{\prime}\right] \ldots\left[x_{p}, B_{p}^{\prime}\right]$. Now both $Q_{1}^{\prime} K^{\prime}$ and $Q_{2}^{\prime} L^{\prime} \epsilon \Delta$, so $k=\ell$ : assume $k>\ell$, then $M \equiv\left[x_{1}, A_{1}^{\prime}\right] \ldots\left[x_{p-k}, A_{p-k}^{\prime}\right] M^{\prime} \equiv\left[x_{1}, B_{1}^{\prime}\right] \ldots\left[x_{p-k}, B_{p-k}^{\prime}\right] \ldots$ $\left[x_{p-\ell}, B_{p-\ell}^{\prime}\right] M^{\prime \prime}$; it follows that $\left[x_{p-\ell}, B_{p-\ell}^{\prime}\right]$ occurs in $M^{\prime}$, hence 
also in $K^{\prime}$; this contradicts the fact that $Q_{1}^{\prime} K^{\prime} \in \Delta$, since we found two binding variables $x_{p-\ell}$ in $Q_{1}^{\prime} K^{\prime}$. It follows that $K^{\prime} \equiv L^{\prime}$; we can take $N \equiv K^{\prime} \equiv L^{\prime}$.

Corollary 7.17. If $\mathrm{QK}, \mathrm{QL} \in \Delta, \mathrm{QK} \geq_{\eta} \mathrm{M}$ and $\mathrm{QL} \geq_{n} \mathrm{M}$, there is a $\mathrm{QN}$ such that $\mathrm{QK} \geq_{\eta} \mathrm{QN}$ and $\mathrm{QL} \geq_{n} \mathrm{QN}$.

Theorem 7.18. If $Q D \in \Delta, Q D \geq_{\eta} Q^{\prime} E, Q \equiv\left[x_{1}, A_{1}\right] \ldots\left[x_{p}, A_{p}\right]$ and $Q^{\prime} \equiv\left[x_{1}, A_{1}^{\prime}\right] \ldots\left[x_{p}, A_{p}^{\prime}\right]$, then $Q D \geq_{n} Q E$.

Proof. Resulting from Th. 7.14 we can find a reduction $\overline{Q D} \geq_{n} Q^{\prime \prime} D^{\prime} z_{n !}^{(k)} Q^{\prime} E$ where $Q^{\prime \prime} D^{\prime} z_{n !}^{(k)} Q^{\prime} E$ is of order $\|Q\|$. Now $Q^{\prime \prime} \equiv\left[x_{1}, A_{1}^{\prime \prime}\right] \ldots\left[x_{p}, A_{p}^{\prime \prime}\right]$, hence $k=0$ (because $Q^{\prime \prime} D^{\prime} \in \Delta$; see the proof of $T h .7 .16)$. Then also $Q D \geq_{\eta} Q E$ by $T h .7 .15$.

We shall now prove a theorem concerning the so-called "postponement of $n$-reductions" for $\Delta$. What we want to prove is that every reduction $K \geq M$ which takes place by means of single-step $\beta^{-}$and $n$-reductions in arbitrary order, can be replaced by a reduction $K \geq_{\beta} L \geq_{\eta} M$, in which all $B$-reductions precede all $n$-reductions.

It is easy to show that each reduction $A \geq_{n}^{\prime} B \geq_{B}^{\prime} C$ can be replaced either by a reduction $A \geq_{B}^{\prime} B^{\prime} \geq_{n}^{r} C$ (where $r \geq 0$ ) or by a reduction $A \geq_{B}^{\prime} B^{\prime} \geq_{B}^{\prime} C$. But this does not suffice to prove the theorem. It is not sure that this process of interchanging $n^{\prime} s$ and $B^{\prime}$ 's terminates for a given reduction $k \geq M$.

In Curry and Feys [3, Ch. 4, $\$$ D2] a compound B-reduction is introduced for the purpose of proving the above mentioned theorem. In our opinion there is an error in their proof (viz*, the case that $R$ is $M_{k} N$ and $L$ is some $M_{j} y_{j}$ for $j \leq k$ is missing). Nevertheless, their idea can be extended in such a manner that the theorem on the postponement of $n$-reductions can be proved. We have carried this out by defining a "compound $\beta$-reduction" $A \geq_{B}^{*} B$ with the property that each reduction $A \geq_{n} B \geq_{B}^{*} C$ can be replaced by a reduction $A \geq_{B}^{*} B^{\prime} \geq_{n}$ C. However, this compound $B$-reduction looks rather complicated. 
Barendregt suggested to us another way of proving the theorem (private communication). He proposed a "nested" n-reduction (which we call k-reduction and denote by $\geq_{K}^{\prime}$ ) with the property that a reduction $A \geq_{K}^{\prime} B \geq_{\beta} C$ can be replaced by a reduction $A \geq_{B} B^{\prime} \geq_{K}^{\prime} C$. The nested character of this $k$-reduction is comparable to that of $\gamma$-reduction discussed in the previous section.

We prefer the latter way of proving because it is easier to understand.

Definition 7.19. Single-step $k$-reduction, denoted by $z_{K}^{\prime}$, is the reflexive relation generated by

(1) If $Q[x, A]\{x\} B \in \Delta, x \neq B$ and $Q B \geq_{k}^{\prime} Q C$, then $Q[x, A]\{x\} B \geq_{k}^{\prime} Q C$.

(2) If $Q\{A\} C \in \Delta, Q A \geq_{K}^{\prime} Q A^{\prime}$ and $Q C \geq_{K}^{\prime} Q C^{\prime}$, then $Q\{A\} C \geq_{K}^{\prime} Q\left\{A^{\prime}\right\} C^{\prime}$.

(3) If $Q[x, A] C \in \Delta, Q A \geq_{K}^{\prime} Q A^{\prime}$ and $Q[x, A] C \geq_{K}^{\prime} Q[x, A] C^{\prime}$, then $Q[x, A] C \geq_{K}^{\prime} Q\left[x, A^{\prime}\right] C^{\prime}$.

We call rule (1) in this definition the rule of elementary single-step k-reduction, rules (2) and (3) the monotony miles for k-reduction.

The following two theorems deal with the relation between $n-$ and k-reduction.

Theorem 7.20. If $K \in \Delta$ and $K \geq_{\eta}^{\prime} L$, then $K \geq_{K}^{\prime} L$.

Proof. Induction on the length of proof of $\mathrm{K} \geq_{\eta}^{\prime} L$.

Theorem 7.21. If $\mathrm{K} \in \Delta$ and $\mathrm{K} \geq \geq_{K}^{\prime} \mathrm{L}$, then $\mathrm{K} \geq_{\eta} \mathrm{L}$.

Proof. Induction on the length of proof of $x \geq_{K}^{1}$ L.

For example, if $K \geq_{K}^{\prime} L$ is $Q[x, A]\{x\} B \geq_{K}^{\prime} Q C$, as a direct consequence of $Q B \geq_{K}^{\prime} Q C$, then by induction $Q B \geq_{\eta} Q C$, and $Q[x, A]\{x\} B \geq_{n}^{\prime}$ $\mathrm{QB} \geq_{n} \mathrm{QC}$.

We shall now prove a number of theorems which are lemmas for the theorem on the postponement of n-reductions (Th. 7.28). 
Theorem 7.22. If $K \in \Delta$ and $K \geq \geq_{K}^{\prime} L$, then $L \in \Delta$.

Proof. Follows from Th. 7.21 and Th. 7.4 .

Theorem 7.23. If $\mathrm{QE} \in \Delta$ and $\mathrm{QE} \geq_{K}^{\prime} \mathrm{Q}[\mathrm{y}, \mathrm{G}] \mathrm{H}$, then $Q E \equiv Q\left[x_{1}, A_{1}\right]\left\{x_{1}\right\}\left[x_{2}, A_{2}\right]\left\{x_{2}\right\} \ldots\left[x_{n}, A_{n}\right]\left\{x_{n}\right\}\left[y, G^{\prime}\right] H^{\prime}$, with $Q G^{\prime} \geq_{K}^{\prime} Q G, Q\left[y, G^{\prime}\right] H^{\prime} \geq_{K}^{\prime} Q\left[y, G^{\prime}\right] H$ and $x_{i} \notin\left[x_{i+1}, A_{i+1}\right] \ldots\left\{x_{n}\right\}\left[y, G^{\prime}\right] H^{*}$.

Proof. Induction on the length of proof of $Q E \geq_{K}^{\prime} Q[y, G] H$. If the latter reduction results from reflexivity, the proof is completed.

(1) Let $Q E \geq_{K}^{\prime} Q[y, G] H$ be $Q^{\prime}[x, A]\{x\} B \geq_{K}^{\prime} Q^{\prime} C$, as a direct consequence of $Q^{\prime} B \geq_{K}^{\prime} Q^{\prime} C$. If $Q^{\prime} \equiv Q Q^{\prime \prime}$, induction yields the proof. If $Q \equiv Q^{\prime}[x, A]$, then $C$ begins with $[x, A]$. This implies that $[x, A]$ occurs in $B$ (since $k$-reduction can only omit abstractors and applicators without influencing the remainder of the expression), which is impossible since $Q E \in \Delta$. So this latter case cannot apply. (2) Let $Q E \geq_{K}^{\prime} Q[y, G] H$ be $Q^{\prime}\{A\} C \geq_{K}^{\prime} Q^{\prime}\left\{A^{\prime}\right\} C^{\prime}$. Then $Q E \equiv Q[y, G] F$. (3) Let $Q E \geq_{K}^{\prime} Q[y, G] H$ be $Q^{\prime}[x, A] C \geq_{K}^{\prime} Q^{\prime}\left[x, A^{\prime}\right] C^{\prime}$, as a direct consequence of $Q^{\prime} A \geq_{K}^{\prime} Q^{\prime} A^{\prime}$ and $Q^{\prime}[x, A] C \geq_{K}^{\prime} Q^{\prime}[x, A] C^{\prime}$. There are the following possibilities: (a) $Q \equiv Q^{\prime},(b) Q \equiv Q^{\prime}[x, A] Q_{1}$ and (c) $Q^{\prime} \equiv Q_{1}$ with $\left\|Q_{1}\right\|>0$. In all three cases the proof is easy.

Theorem 7.24. Let $\mathrm{QA}$ and $\mathrm{Q}[\mathrm{x}, \mathrm{B}] \mathrm{C} \in \Delta, \mathrm{QA} \geq_{K}^{\prime} \mathrm{QA}^{\prime}$ and $\mathrm{Q}[\mathrm{x}, \mathrm{B}] \mathrm{C} \geq_{K}^{\prime}$ $Q[x, B] C^{\prime}$. Then $Q(x:=A) C \geq_{K}^{\prime} Q\left(x:=A^{\prime}\right) C^{\prime}$.

Proof. Induction on $|c|$. If $C \equiv \tau, C \equiv x$ or $C \equiv y \not x$, the proof is easy.

(1) Let $C \equiv[y, E] F$. There are two possible cases:

(a) $Q[x, B] C \geq_{K}^{\prime} Q[x, B] C^{\prime}$ is $Q[x, B][y, E] F \geq_{K}^{\prime} Q[x, B]\left[y, E^{\prime}\right] F^{\prime}$, as a direct consequence of $Q[x, B] E \geq_{K}^{\prime} Q[x, B] E^{\prime}$ and $Q[x, B][y, E] F \geq_{K}^{\prime}$ $Q[x, B][y, E] F^{\prime}$. By induction: $Q(x:=A) E \geq_{K}^{\prime} Q\left(x:=A^{\prime}\right) E^{\prime}$, and $\mathrm{Q}[\mathrm{y},(\mathrm{x}:=\mathrm{A}) \mathrm{E}](\mathrm{x}:=\mathrm{A}) \mathrm{F} \geq_{K}^{\prime} \mathrm{Q}[\mathrm{y},(\mathrm{x}:=\mathrm{A}) \mathrm{E}]\left(\mathrm{x}:=\mathrm{A}^{\prime}\right) \mathrm{F}^{\prime}$ (the latter because $\left.Q[y,(x:=A) E][x, B] F \geq_{K}^{\prime} Q[y,(x:=A) E][x, B] F^{\prime}\right)$. Hence $Q(x:=A) C \geq_{K}^{\prime}$ $Q\left(x:=A^{\prime}\right) C^{\prime}$. 
(b) $Q[x, B] C \geq_{K}^{\prime} Q[x, B] C^{\prime}$ is $Q[x, B][y, E]\{y\} G \geq_{K}^{\prime} Q[x, B] G^{\prime}$, as a direct consequence of $Q[x, B] G \geq_{K}^{\prime} Q[x, B] G^{\prime}$. By induction $Q(x:=A) G \geq_{K}^{\prime}$ $Q\left(x:=A^{\prime}\right) G^{\prime}$, so $Q(x:=A)[y, E]\{y\} G \equiv Q[y,(x:=A) E]\{y\}(x:=A) G \geq_{K}^{\prime}$ $Q\left(x:=A^{\prime}\right) G^{\prime}$.

(2) Let $C \equiv\{E\} F$. Then $Q[x, B] C \geq_{K}^{\prime} Q[x, B] C^{\prime}$ is $Q[X, B]\{E\} F \geq_{K}^{\prime}$ $Q[x, B]\left\{E^{\prime}\right\} F^{\prime}$, as a direct consequence of $Q[x, B] E \geq_{K}^{\prime} Q[x, B] E^{\prime}$ and $Q[x, B] F \geq_{K}^{\prime} Q[x, B] F^{\prime}$. The theorem results from the induction. (Note that $Q[x, B] C \geq_{K}^{\prime} Q[x, B] C^{\prime}$ cannot be $Q[x, B]\{x\} G \geq_{K}^{\prime} Q G^{\prime}$. )

Theorem 7.25. Let $A \in \Delta$ and $A \geq_{K}^{\prime} B \geq_{B}^{\prime}$ C. Then $A \geq_{\beta} B^{\prime} \geq_{K}^{\prime} C$. Proof. Induction on the length of proof of $A \geq_{K}^{\prime} B$. If the last derivation step results from reflexivity, nothing remains to be proved.

(1) Let $A \geq_{K}^{\prime} B$ be $Q[x, D]\{x\} E \geq_{K}^{\prime} Q E^{\prime}$ as a direct consequence of $Q E \geq_{K}^{\prime} Q E^{\prime}$, and let $B \geq_{B}^{\prime} C$ be generated by $Q^{\prime}\{F\}[y, G] H \geq_{B}^{\prime} Q^{\prime}(y:=F) H$. The following cases may apply:

(a) $\{F\}[y, G] H \subset Q$. There is clearly a reduction $A \geq_{B} B^{\prime} \geq_{K}^{\prime} C$.

(b) $\{F\}[y, G] H=E^{\prime}$. Let $B \geq_{B}^{\prime} C$ be $Q E^{\prime} \geq_{B}^{\prime} Q E^{\prime \prime \prime}$, then by induction there is a reduction

$Q E \geq_{\beta} Q E^{\prime \prime} \geq_{K}^{\prime} Q E^{\prime \prime \prime}$, hence $Q[x, D]\{x\} E \geq_{\beta} Q[x, D]\{x\} E^{\prime \prime} \geq_{K}^{\prime} Q E^{\prime \prime}$.

(2) Let $A \geq_{K}^{\prime} B$ be $Q\{D\} E Z_{K}^{\prime} Q\left\{D^{\prime}\right\} E^{\prime}$ as a direct consequence of $Q D \geq_{K}^{\prime} Q D^{\prime}$ and $Q E \geq_{K}^{\prime} Q E^{\prime}$, and $1 e t B \geq_{\beta}^{\prime} C$ be generated by $Q^{*}\{F\}[y, G] H \geq_{B}^{\prime} Q^{\prime}(y:=F) H$.

The following cases may apply:

(a) $\{\mathrm{F}\}[y, G] \mathrm{H} \subset \mathrm{Q}$. Clearly $A \geq_{B} \mathrm{~B}^{\prime} \geq_{K}^{\prime} \mathrm{C}$.

(b) $\{F\}[y, G] H \equiv\left\{D^{\prime}\right\} E^{\prime}$. Then $Q E^{\prime} \equiv Q[y, G] H$, so $Q E \equiv Q\left[x_{1}, A_{1}\right]\left\{x_{1}\right\} \ldots\left[x_{n}, A_{n}\right]\left\{x_{n}\right\}\left[y, G^{\prime}\right] H^{\prime}$, with $Q G^{\prime} \geq_{K}^{\prime} Q G$, $Q\left[y, G^{\prime}\right] H^{\prime} \geq_{K}^{\prime} Q\left[y, G^{\prime}\right] H$ and $x_{i} \notin\left[x_{i+1}, A_{i+1}\right] \ldots\left\{x_{n}\right]\left[y, G^{\prime}\right] H^{\prime}$ by Th. 7.23. Then $Q\{D\} E \geq_{B} Q(y:=D) H^{\prime}$. By Th. 7.24:Q(y:=D) $H^{\prime} \geq_{K}^{\prime}$ $\mathrm{Q}(\mathrm{y}:=\mathrm{F}) \mathrm{H} \equiv \mathrm{C}$.

(c) $\{F\}[y, G] H \subset D^{\prime}$. Then $C \equiv Q\left\{D^{\prime \prime \prime}\right\} E^{\prime}$ with $Q D^{\prime} \geq_{B}^{\prime} Q D^{\prime \prime \prime}$, and by induction $Q D \geq_{B} Q D^{\prime \prime} \geq_{K}^{\prime} Q D^{\prime \prime}$, hence $Q\{D\} E \geq_{B} Q\left\{D^{\prime \prime}\right\} E \geq_{K}^{\prime} Q\left\{D^{\prime \prime}\right\} E^{\prime} \equiv C$. (d) $\{F\}[y, G] H \subset E^{\prime}$. Then $C \equiv Q\left\{D^{\prime}\right\} E^{\prime \prime \prime}$ with $Q E^{\prime} \geq_{B}^{\prime} Q E^{\prime \prime \prime}$, and by

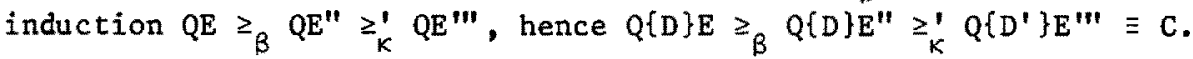


(3) Let $A \geq_{K}^{\prime} B$ be $Q[x, D] E \geq_{K}^{\prime} Q\left[x, D^{\prime}\right] E^{\prime}$ as a direct consequence of $Q D z_{K}^{\prime} Q^{\prime}$ and $Q[x, D] E z_{K}^{\prime} Q[x, D] E^{\prime}$, and let again $B z_{B}^{\prime} C$ be generated by $Q^{\prime}\{F\}[y, G] H \geq_{\beta}^{\prime} Q^{\prime}(y:=F) H$.

The following cases may apply:

(a) $\{F\}[y, G] H \subset Q$. Clearly $A \geq_{B} B^{\prime} \geq_{K}^{\prime} C$.

(b) $\{F\}[y, G] H \subset D^{\prime}$. Then $C \equiv Q\left[x, D^{\prime \prime}\right] E^{\prime}$ with $Q D^{\prime} \geq_{B}^{\prime} Q D^{\prime \prime \prime}$.

By induction: $Q D \geq_{B} Q D^{\prime \prime} \geq_{K}^{\prime} Q D^{\prime \prime \prime}$, so $Q[x, D] E \geq_{B} Q\left[x, D^{\prime \prime}\right] E \geq_{K}^{\prime}$ $Q\left[x, D^{\prime \prime \prime}\right] E^{\prime}$ (where we require the lemma: $Q[x, D] E \geq_{K}^{\prime} Q[x, D] E^{\prime}$, then $\left.Q\left[x, D^{\prime \prime}\right] E \geq_{K}^{\prime} Q\left[x, D^{\prime \prime}\right] E^{\prime}\right)$.

(c) $\{F\}[y, G] H \subset E^{\prime}$. Then $C \equiv Q\left[x, D^{\prime}\right] E^{\prime \prime \prime}$.

Also: $Q[x, D] E^{\prime} \geq_{B}^{\prime} Q[x, D] E^{\prime \prime \prime}$, so by induction $Q[x, D] E \geq_{\beta} Q[x, D] E^{\prime \prime} \geq_{K}$ $Q[x, D] E^{\prime \prime \prime}$. Hence $Q[x, D] E \geq_{B} Q[x, D] E^{\prime \prime} \geq_{K} Q\left[x, D^{\prime}\right] E^{\prime \prime \prime} \equiv C$.

Theorem 7.26. Let $A \in \Delta$ and $A \geq_{K}^{\prime} B \geq_{B}^{P} C$. Then $A \geq_{B} B \geq_{K}^{\prime} C$. Proof. Induction on $\mathrm{p}$, using the previous theorem.

Theorem 7.27. Let $A \in \Delta$ and let $A \geq C$ by means of a number of single-step $\kappa-$ and $\beta$-reductions in arbitrary order. Then there is a reduction $A \geq_{B} B \geq_{K} C$.

Proof. Induction on the number of single-step $k$-reductions in $A \geq C$. If this number is zero, the proof is completed. Else, let $A \geq C$ be $A \geq A^{t} \geq_{K}^{\prime} B \geq_{B}^{P} C$. Apply the previous theorem, obtaining $A \geq A^{\prime} \geq_{B} B^{\prime} \geq_{K}^{\prime} C$, and apply the induction on $A \geq A^{\prime} \geq_{B} B^{\prime}$.

Theorem 7.28. Let $\mathrm{A} \in \Delta$ and let $\mathrm{A} \geq \mathrm{C}$ by means of a number of single-step $n$ - and $B$-reductions in arbitrary order. Then there is a reduction $A \geq_{B} B \geq_{\eta}$.

Proof. Since each n-reduction can be considered as a k-reduction (Th. 7.20), we can apply the previous theorem, obtaining $A \geq_{B} B \geq_{K} C$. But $B \geq_{K} C$ implies $B \geq_{\eta} C$ (Th. 7.21), so $A \geq_{B} B \geq_{\eta}$ C.

The remainder of this section will concern (general) reduction, defined as a sequence of single-step $\alpha-, \beta-$ and $n-r e d u c t i o n s$. 
Definition 7.29. Single-step reduction (denoted by $\geq^{\prime}$ ) is the relation obeying:

$A \geq^{\prime} B$ if and only if $A \geq_{\alpha}^{\prime} B, A \geq_{B}^{\prime} B$ or $A \geq_{\eta}^{\prime} B$.

Definition 7.30. Reduction (or general reduction, denoted by $\geq$ ) is the reflexive and transitive closure of single-step reduction.

Theorem 7.31. The monotony rules hold for reduction.

Proof. Use Th. 5.15 and Th. 7.8.

We shall prove a theorem (Th. 7.33) which expresses that the $Q$ is in a certain sense irrelevant in a reduction $Q C \geq Q E$ : it can be replaced by any $\mathrm{P}$ such that $\mathrm{PC}$ and $\mathrm{PE} \in \Delta$. This corresponds with general usage in lambda-calculus to define reduction for expressions which may contain free variables. Our choice to define reductions inside $\Delta$ is apparently not in disagreement with that general usage.

Theorem 7.32. If $Q C \in \Delta$ and $Q C \geq Q E$ by means of $B-$ and $n$-reductions, there is a reduction $Q C \geq_{B} Q D \geq_{\eta} Q E$.

Proof. There is a reduction $Q C \geq_{B} K \geq_{n} Q E$ by Th. 7.28. Now by $T h .5 .12: K \equiv Q^{\prime} D$ with $\|Q\|=\left\|Q^{\prime}\right\|$.

If $Q \equiv\left[x_{1}, A_{1}\right] \ldots\left[x_{p}, A_{p}\right]$, then $Q^{\prime} \equiv\left[x_{1}, A_{1}^{\prime}\right] \ldots\left[x_{p}, A_{p}^{\prime}\right]$, so by Th. 7.18: $Q^{\prime} D \geq_{n} Q^{\prime} E$. From Th. 5.21: $Q C \geq_{B} Q D$, and from Th. 7.9: $\mathrm{QD} \geq_{\eta} \mathrm{QE}$.

Theorem 7.33. If $\mathrm{QC}, \mathrm{PC}$ and $\mathrm{PE} \in \Delta$, and $\mathrm{QC} \geq \mathrm{QE}$, then $\mathrm{PC} \geq \mathrm{PE}$. Proof. See Th. 7.32, Th. 5.17 and Th. 7.9.

Reduction is a non-symetric relation between expression in $\Delta$, which is reflexive and transitive. We shall define lambda-equivalence. The definition of beta-equivalence was given in Def. 5.22. In Th. 7.35 we shall prove that beta-equivalence is the symetric closure of beta-reduction. 
Definition 7.34. Let $A$ and $B \in \Delta$. We call $A$ Zambda-equivalent to $B$ (denoted: $A \sim B$ ) if there is an expression $C$ such that $A \geq C$ and $\mathrm{B} \geq \mathrm{C}$.

Theorem 7.35. Beta-equivalence is reflexive, symmetric and transitive.

Proof. Reflexivity and symmetry are trivial. Transitivity follows from Th. 6.43 (CR for $B$-reduction): let $A \sim_{B} B$ and $B \sim_{B} C$, then there are $D$ and $E$ such that $A \geq_{B} D, B \geq_{B} D, B \geq_{B} E$ and $C \geq_{B} E$. Moreover, there is an $F$ such that $D \geq_{B} F$ and $E \geq_{B} F$ (Th. 6.43), so $A \geq_{B} F$ and $C \geq_{\beta} F$. Hence $A \sim_{\beta} C$.

Unfortunately, there is no similar theorem for lambda-equivalence. Of course lambda-equivalence is symmetric and reflexive, but not necessarily transitive. The reason for this is that $C R$ does not hold for (general) reduction: for example, let $K \geq L$ and $K \geq M$, let $K \equiv Q[x, A]\{x\}[y, B] C$ where $x \notin[y, B] C$, let $K \geq I$ be $Q[x, A]\{x\}[y, B] C \geq \geq_{n} Q[y, B] C$ and 1 et $K \geq M$ be $Q[x, A]\{x\}[y, B] C \geq_{B}$ $Q[x, A](y:=x) C\left(\geq_{\alpha} Q[y, A] C\right)$. Now we cannot in general find an $N$ such that $L \geq N$ and $M \geq N$, since we know nothing concerning a relation between $A$ and $B$.

We note the following. We can embed ordinary lambda-calculus into $\Delta$, since there is a one-to-one correspondence between expressions from lambda-calculus and those expressions in $\Delta$ in which only abstractors of the form $[x, \tau]$ occur. If we restrict ourselves in $\Delta$ to the latter expressions, the example above changes into $K \equiv Q[x, \tau]\{x\}[y, \tau] C, L \equiv Q[y, \tau] C$ and $M \geq_{\alpha} Q[y, \tau] C$. Now there is no problem as regards CR. Indeed, in lambda-calculus the ChurchRosser property holds (see Barendregt [1, Appendix II]).

The following theorem expresses that lambda-equivalence of $Q K$ and $Q L$ implies the existence of an $N$ such that $Q K \geq Q N$ and $Q L \geq Q N$ or, otherwise stated the abstractor chain $Q$ can remain unaffected. 
Theorem 7.36. Let $\mathrm{QK}$ and $\mathrm{QL} \in \Delta$. If $\mathrm{QK} \sim \mathrm{QL}$, there exists an $\mathrm{N}$ such that $\mathrm{QK} \geq \mathrm{QN}$ and $\mathrm{QL} \geq \mathrm{QN}$.

Proof. There must be an $M: Q K \geq M$ and $Q L \geq M$. By postponement of $n$-reductions we obtain reductions $Q K \geq_{\beta} M_{1} \geq_{\eta} M$ and $Q L \geq_{\beta} M_{2} \geq_{\eta} M$. Th. 5.12 implies that $M_{1} \equiv Q_{1} K^{\prime}, M_{2} \equiv Q_{2} L^{\prime},\|Q\|=\left\|Q_{1}\right\|=\left\|Q_{2}\right\|$. Then, according to Th. 5.21, we also have $Q K \geq_{B} Q K^{\prime} \geq_{B} Q_{1} K^{\prime} \geq_{\eta} M$ and $Q L \geq_{\beta} Q^{\prime} \geq_{\beta} Q_{2} L^{\prime} \geq_{\eta} M$. It is easy to show that $Q_{1}$ and $Q_{2}$ have the form as required in $T h .7 .16$, hence there is an $N$ such that $Q_{1} K^{\prime} \geq_{\eta} Q_{1} N$ and $Q_{2} L^{\prime} \geq_{\eta} Q_{2} N$. From Th. 7.9 it follows that $Q^{\prime} \geq_{\eta} Q N$ and $\mathrm{QL}^{\prime} \geq_{\eta} \mathrm{QN}$. So $\mathrm{QK} \geq \mathrm{QN}$ and $\mathrm{QL} \geq \mathrm{QN}$.

The monotony rules also hold for lambda-equivalence:

Theorem 7.37 .

(a) If $Q C, Q D, Q\{A\} C, Q\{A\} D \in \Delta$ and $Q C \sim Q D$, then $Q\{A\} C \sim Q\{A\} D$.

(b) If $Q C, Q D, Q[x, A] C, Q[x, A] \Phi \in \Delta$ and $Q C \sim Q D$, then $Q[x, A] C \sim Q[x, A] D$.

(c) If $Q A, Q B, Q\{A\} C, Q\{B\} C \in \Delta$ and $Q A \sim Q B$, then $Q\{A\} C \sim Q\{B\} C$.

(d) If $Q A, Q B, Q[x, A] C, Q[x, B] C \in \Delta$ and $Q A \sim Q B$, then $Q[x, A] C \sim Q[x, B] C$.

Proof. See Th. 7.36 and Th. 7.33.

Theorem 7.38. If $Q C, Q D, P C$ and $P D \in \Delta$ and $Q C \sim Q D$, then $P C \sim P D$. Proof. Th. 7.36 and Th. 7.33.

\section{8. TYPE AND DEGREE}

The notions introduced in the preceding sections are from lambda-calculus (as reduction, lambda-equivalence) or applicable to lambda-calculus (factors, bound expressions), since the types played no essential rôle.

We shall now look into the typing of an expression in $\Delta$. With every $A \in \Delta$ for which Tail $A \neq \tau$ we define a type, denoted as Typ A, as follows: 
Definition 8.1. Let $A \in \Delta$ and Tail $A \equiv x$, so $A \equiv P_{1}[x, B] P_{2} x$. Then Typ $A \equiv P_{1}[x, B] P_{2} F r B$.

Informally speaking, we may say that $B$ is the type of $x$ in the above expression. Note, however, that we allow Typ to operate only on expressions in $\Delta$.

Theorem 8.2. If $A \in \Delta$ and Tail $A \neq \tau$, then Typ $A \in \Delta$.

Proof. Let $A \equiv P_{1}[x, B] P_{2} x$ and let $A \mid x \equiv Q_{1}[x, B] Q_{2} x$.

We prove that Typ $A$ is a bound expression. All non-binding variables in $P_{1}[x, B] P_{2}$ are clearly also bound in Typ A. Consider a non-binding variable $z \subset \operatorname{FrB} \subset Q_{1}[x, B] Q_{2} F r B$. There is a corresponding $y \subset B$, and $A \mid y \equiv Q_{1} Q_{3} y$. So Typ $A \mid z \equiv Q_{1}[x, B] Q_{2} Q_{3}^{\prime} z$ where $Q_{3}^{\prime} z$ is a renovation of $Q_{3} \mathrm{y}$

Case 1: if $y$ was bound in $A / y$ by a binding variable in $Q_{3}, z$ is bound in Typ $A / z$ by the corresponding binding variable in $Q_{3}^{\prime}$.

Case 2: if $y$ was bound in $A \mid y$ by a binding variable in $Q_{1}, z \equiv y$ is still bound by the same binding variable in $Q_{1}$ since all binding variables of $[x, B] Q_{2} Q_{3}^{*}$ are different from $y$.

So Typ $A$ is bound. Clearly Typ $A$ is also distinctly bound by the renovation of $B$.

We define repeated application of Typ inductively as follows:

Definition 8.3. Let $A \in \Delta$. Then $\operatorname{Typ}^{0} A \equiv A$; if $\operatorname{Typ}^{n-1} A$ is defined for $n \geq 1$ and if $\operatorname{Tai} 1 \operatorname{Typ}^{n-1} A \neq \tau$, then $\operatorname{Typ}^{n} A \equiv \operatorname{Typ}\left(\operatorname{Typ}^{n-1} A\right)$.

If $A \in \Delta$ and $\operatorname{Typ}^{\mathrm{n}} \mathrm{A}$ is defined, we call n permissible for $\mathrm{A}$ ( $n=0$ is always permissible for $A \in \Delta$ ).

Theorem 8.4. If $A \in \Delta$ and $A \geq_{\alpha} B$, then $\operatorname{Typ}^{n} A \geq_{\alpha} \operatorname{Typ}^{n} B$ for al1 $n$ permissible for $A$ and $B$.

Proof. It is sufficient to prove: if $A \geq_{\alpha}^{\prime} B$ and Tail $A \not \tau$, then Typ $A \geq_{\alpha}$ Typ B. The latter proof is easy. 
With each expression $A$ in $\Delta$ we define a degree, denoted Deg A:

\section{Definition 8.5.}

(1) If $\mathrm{A} \in \Delta$ and $\operatorname{Tail} \mathrm{A} \equiv \tau$, then $\operatorname{Deg}(\mathrm{A})=1$.

(2) If $A \in \Delta$, Tail $A \equiv x$ and $A \equiv P_{1}[x, B] P_{2} x$, then $\operatorname{Deg}(A)=\operatorname{Deg}\left(P_{1} B\right)+1$.

Induction on the length of $A$ shows that $\operatorname{Deg}(A)$ is welldefined by Def. 8.5. Clearly Deg $A=1$ if and only if Tail $A \equiv \tau$.

We shall now prove a number of theorems, leading to the theorem: if Tail A $\neq \tau$, then Deg $A=\operatorname{Deg} \operatorname{Typ} A+1$ (Th. 8.12). We could have taken this property as a definition of Deg. In that case, however, the well-definedness of Deg would have been harder to prove.

Theorem 8.6. If $\mathrm{PC} \in \Delta$ and $\mathrm{P}\{\mathrm{K}\} \mathrm{C} \in \Delta$, then Deg $\mathrm{PC}=\operatorname{Deg} \mathrm{P}\{\mathrm{K}\} \mathrm{C}$. Proof. Induction on $|P C|$.

Corollary 8.7. If $A \in \Delta$ and $A \equiv P C$, then $\operatorname{Deg} A=\operatorname{Deg}(A \mid C)$.

Corollary 8.8. If $A \in \Delta$, Tail $A \equiv x$ and $A \mid x \equiv Q_{1}[x, B] Q_{2} x$, then $\operatorname{Deg} A=\operatorname{Deg} Q_{1} B+1$.

Theorem 8.9. If $\mathrm{PC} \epsilon \Delta$ and $\mathrm{P}[\mathrm{x}, \mathrm{K}] \mathrm{C} \in \Delta$, then Deg $\mathrm{PC}=\operatorname{Deg} \mathrm{P}[\mathrm{x}, \mathrm{K}] \mathrm{C}$. Proof. By Th. 3.8: $\mathrm{x} \notin \mathrm{C}$. The rest of the proof follows from induction on $|P C|$.

Theorem 8.10. If $\mathrm{PC} \in \Delta$ and $\mathrm{PP}^{\prime} \mathrm{C} \in \Delta$, then Deg PC $\equiv$ Deg $P P^{\prime} \mathrm{C}$. Proof. Induction on $\left\|P^{\prime}\right\|$, using Th. 8.6 and Th. 8.9.

Theorem 8.11. If $A \in \Delta$ and $A \geq{ }_{\alpha} B$, then Deg $A=\operatorname{Deg} B$. Proof. Take $A \geq \geq_{\alpha}^{\prime} B$; induction on $|A|$. 
Theorem 8.12. If $A \in \Delta$ and Tail $A \not \tau$, then Deg Typ $A=\operatorname{Deg} A-1$. Proof. Let Tail $A \equiv x$ and $A \equiv P_{1}[x, C] P_{2} x$, so Typ $A \equiv P_{1}[x, C] P_{2} F r C$. Then, $P_{1}$ FrC $\in \Delta$ and Deg $P_{1} F r C=\operatorname{Deg} P_{1} C$ by Th. 4.5 and Th. 8.11. By Th. 8.10: Deg $P_{1}$ FrC = Deg Typ A. So Deg $A=\operatorname{Deg} P_{1} C+1=\operatorname{Deg} \operatorname{Typ} A+1$.

Corollary 8.13. If $A \in \Delta$, then Tail(Typ $\left.{ }^{\text {Deg } A-1} A\right) \equiv \tau$.

This optimal exponent of Typ with a certain $A \epsilon \Delta$ is of special importance. We shall introduce an abbreviation:

Definition 8.14. If $A \in \Delta$, then Typ ${ }^{*} \equiv \operatorname{Typ}^{\text {Deg } A-1}$ A.

We stress that the asterisk replaces an exponent $\mathrm{n}$ dependent upon A. Moreover, note that Typ is a partial function on $\Delta$, but Typ $^{*}$ is a total function on $\Delta$.

We proceed with a number of theorems on Typ ${ }^{n}$, Typ $^{*}$ and Deg:

Theorem 8.15. If $A \in \Delta, \operatorname{Deg} A=1$ and $A \geq B$, then $\operatorname{Deg} B=1$.

Theorem 8.16. If $\mathrm{PC} \in \Delta$, then for permissible $\mathrm{n}$ Typ ${ }^{\mathrm{n}} \mathrm{PC} \equiv \mathrm{PC}^{\prime}$; in particular Typ ${ }^{\star} P C \equiv P C^{\prime \prime}$.

Theorem 8.17. If $\mathrm{PC} \in \Delta, \mathrm{PP}^{\prime} \mathrm{C} \in \Delta$, and for a permissible $\mathrm{n}$ Typ ${ }^{\mathrm{n}} \mathrm{PC} \equiv$ $P C^{\prime}$, then $n$ is permissible for $P P^{\prime} C$, and Typ ${ }^{n} P P^{\prime} C \geq_{a} P P^{\prime} C^{\prime}$. Proof. It is sufficient to assume Tail $P C \not \tau$ and $n=1$. Let Tai1 $P C \equiv x$ and $(P C) \mid x \equiv Q_{1}[x, B] Q_{2} x$, then $\left(P P^{\prime} C\right) \mid x \equiv Q_{1}^{\prime}[x, B] Q_{2}^{\prime} x$, and $[x, B]$ appears in either $P$ or $C$. The remainder follows.

Theorem 8.18. If $\mathrm{PC} \in \Delta, \mathrm{PP}^{\prime} \mathrm{C} \in \Delta$ and for a permissible $\mathrm{n}$ Typ $\mathrm{PP}^{\prime} \mathrm{C} \equiv$ $\mathrm{PP}^{\prime} \mathrm{C}^{\prime}$, then $\mathrm{n}$ is permissible for $\mathrm{PC}$ and $\operatorname{Typ}^{\mathrm{n}} \mathrm{PC} \geq_{\alpha} \mathrm{PC}^{\dagger}$.

Proof. Similar to the previous proof. 


\section{CHAPTER III, THE FORMAL SYSTEM $\Lambda$}

\section{1. LEGITIMATE EXPRESSIONS}

The "meaning" of $\{A\} B$ is the application of function $B$ to argument A. So far this application was unrestricted: any expression could serve as an argument. Besides, it was of no interest whether $B$ really was a function or not.

In the formal system $\Lambda$, which we shall introduce in this chapter, we only admit the expressions of $\Delta$ which obey the applicability condition. (For an informal introduction of the applicability condition: see section 1.4.) We call this kind of expressions legitimate expressions.

Since $\Lambda$ is a part of $\Delta$, we again provide expressions with abstractor chains $Q$, as we did with expressions in $\Delta$ (cf. the beginning of Section 1.6). We begin with the definitions of function, domain and applicability with respect to an abstractor chain Q:

Definition 1.1* Let $Q B \in \Delta$. We call $Q B$ a $Q$-function if there are $x$, $K$ and $L$ such that $T y{ }^{*} Q B \geq Q[x, K] L$. The expression $Q K$ is called a $\mathrm{Q}$-domain of $\mathrm{QB}$.

Definition 1.2. The expression $Q B$ is called Q-applicable to $Q A$ if $Q B$ is a $Q$-function with $Q$-domain $Q K, \operatorname{Deg} Q A>1$ and Typ $Q A \geq Q$. In that case $Q\{A\} B$ is a legitimate $Q-c p p$ lication of $Q B$ to $Q A$.

The formal system $\Lambda$ is inductively defined by:

\section{Definition 1.3.}

(1) $\tau \in \Lambda$.

(2) If $\mathrm{QA} \in \Lambda$ and if $\mathrm{x}$ does not occur in $\mathrm{QA}$, then $\mathrm{Q}[\mathrm{x}, \mathrm{A}] \mathrm{x} \in \Lambda$ and $Q[x, A] \tau \in \Lambda$.

(3) If $Q A$ and $Q y \in A$, if $x$ does not occur in $Q A$ and if $x \neq y$, then $\mathrm{Q}[\mathrm{x}, \mathrm{A}] \mathrm{y} \in \mathrm{A}$.

(4) If $Q A$ and $Q B \in A$, if the binding variables in $A$ and $B$ are distinct and if $Q B$ is $Q$-applicable to $Q A$, then $Q\{A\} B \in \Lambda$. 
The only difference to the (second) definition of $\Delta$ as given by Th. II.3.10 lies in the applicability condition in (4): QB must be Q-applicable to $Q A$, i.e. Typ ${ }^{*} \mathrm{QB} \geq \mathrm{Q}[\mathrm{y}, \mathrm{K}] \mathrm{L}$ and Typ $\mathrm{QA} \geq \mathrm{QR}$. These reductions are defined for expressions in $\Delta$ (cf. the following Th. 1.4 and Th. II.8.2). Note that the applicability condition does not state that the reductions mentioned concern expressions in $A$ only.

The applicability condition has the powerful consequence that all expressions in $\Lambda$ normalize (cf. Section I.2), which we shall prove later in this chapter, whereas in the wider system $\Delta$ normalization is not guaranteed.

Theorem 1.4. If $A \in \Lambda$, then $A \in \Delta$.

Proof. Induction on the length of proof of $A \in \Lambda$.

Restricting ourselves to $\alpha$ - and $\beta$-reductions, we can weaken the applicability condition in the sense that we replace $\geq$ by $\sim$ :

Theorem 1.5. If $\mathrm{QA}$ and $\mathrm{QB} \in \Lambda, Q[y, K] \mathrm{L} \in \Delta$, Typ ${ }^{*} \mathrm{QB} \sim_{B} \mathrm{Q}[\mathrm{y}, \mathrm{K}] \mathrm{L}$, Typ $Q A \sim_{B} Q K$ and if the binding variables in $A$ and $B$ are distinct, then $Q\{A\} B \in \Lambda$.

Proof. Let Typ ${ }^{*} Q B \equiv Q B^{\prime}$ (Th. II.8.16). Since $Q B^{*} \sim_{B} Q[y, K] L$, there is an $M$ such that $Q B^{\prime} \geq_{B} Q M$ and $Q[y, K] L \geq_{B} Q M$ (Th. II.5.12 and Th. II.5.16). From Th. II.5.16 and Th. II.5.20: $Q M \equiv Q\left[y, K^{\prime}\right] L^{\prime}$ such that $\mathrm{QK} \geq_{B} \mathrm{QK}^{\prime}$.

Let Typ $Q A \equiv Q^{\prime}$. Since $Q A^{\prime} \sim_{B} Q K$, there is a $K^{\prime \prime}$ such that $\mathrm{QA}^{\prime} \geq_{\beta} \mathrm{QK}^{\prime \prime}$ and $\mathrm{QK} \geq_{B} \mathrm{QK}^{\prime \prime}$. Hence (Church-Rosser theorem for $\beta-$ reduction, Th. II.6.43) $\mathrm{QK}^{\prime} \sim \mathrm{QK}^{\prime \prime}$, so there is a $\mathrm{K}^{\prime \prime \prime}: \mathrm{QK}^{\prime} \geq_{B} \mathrm{QK}^{\prime \prime \prime}$ and $\mathrm{QK}^{\prime \prime} \geq_{B} \mathrm{QK}^{\prime \prime \prime}$. Also $\mathrm{Q}\left[\mathrm{y}, \mathrm{K}^{\prime}\right] \mathrm{L}^{\prime} \geq_{B} \mathrm{Q}\left[\mathrm{y}, \mathrm{K}^{\prime \prime \prime}\right] \mathrm{L}^{\prime}$.

Resuming: Typ ${ }^{*} Q B \geq_{B} \cdot Q\left[y, K^{\prime \prime \prime}\right] L^{\prime}$ and Typ $Q A \geq Q K^{\prime \prime}$. So $Q\{A\} B \in \Lambda$.

Note that the above theorem does not hold if we use lambdaequivalence $(\sim)$ instead of B-equivalence $\left(\sim_{\beta}\right)$. Let $Q A \in \Lambda$ and 
Typ $Q A \equiv Q A^{\prime}$. Let $Q B \in \Lambda$ for some $B$. Then Typ ${ }^{\star} Q B \equiv Q B^{\prime} \sim Q\left[y, A^{\prime}\right]\{y\} B^{\prime}$ for some fresh $y$, since $Q\left[y, A^{\prime}\right]\{y\} B^{\prime} \geq_{\eta} Q B^{\prime}$. If the above theorem were to hold with $\sim$ instead of $\sim_{\beta}$, it would follow that $Q\{A\} B \in \Lambda$. Note that $A$ and $B$ are arbitrary. This can clearly not generally be the case. As a counterexample, take $Q \equiv[x, \tau], A \equiv B \equiv x$. Then $Q\{A\} B \equiv$ $[x, \tau]\{x\} x$, which does not belong to $\Lambda$.

We shall prove a number of theorems concerning $\Lambda$.

Theorem 1.6. If $A \in \Lambda$ and $A \geq_{\alpha} B$, then $B \in \Lambda$.

As with $\Delta$, it holds for $\Lambda$ that, given $K \epsilon \Lambda$, only one of the derivation steps in Def. 1.3 can yield $K \in \Lambda$ as a conclusion (unique $\Lambda$-constructibility).

Theorem 1.7. If $Q\{A\} B \in \Lambda$, then $Q A$ and $Q B \in \Lambda$.

Proof. Follows from the unique $\Lambda$-constructibility.

Theorem 1.8. If $\mathrm{Q}[\mathrm{x}, \mathrm{A}] \mathrm{B} \in \Lambda$, then $\mathrm{QA} \in \Lambda$.

Proof. Induction on $|B|$, using the unique $\Lambda$-constructibility. Let $B \equiv\left[y_{1}, B_{1}\right] \ldots\left[y_{k}, B_{k}\right] P s$, where $\left.P \not z, E\right] P^{\prime}$, and $s \equiv \tau$, $\mathrm{s} \equiv \mathrm{y} \equiv \mathrm{x}$ or $\mathrm{s} \equiv \mathrm{x}$.

case $1 . P \equiv \emptyset, k=0$. Then $Q A \in \Lambda$ from rule (2) of Def. 1.3 for all possible s.

case 2. $P \equiv \emptyset, k \geq 1$. Then $Q[x, A]\left[y_{1}, B_{1}\right] \ldots\left[y_{k-1}, B_{k-1}\right] B_{k} \in \Lambda$ from rule (2) or (3), so QA $\in \Lambda$ by induction. case 3. $P \equiv\{E\} P^{\prime}$. Then $Q[x, A]\left[y_{1}, B_{1}\right] \ldots\left[y_{k}, B_{k}\right] E \in \Lambda$ by Th. 1.7, hence $Q A \in \Lambda$ by induction.

Theorem 1.9. If $Q A \in \Lambda$, then $Q \tau \in \Lambda$.

Proof. Induction on $|A|$. If $A \equiv \tau$, there is nothing to prove. If $A \equiv x$, then $Q \equiv Q_{1}[y, B]$ or $Q \equiv Q_{1}[x, B]$. In both cases $Q_{1} B \in \Lambda$, so also $Q \tau \in \Lambda$. If $A \equiv\{B\} C$ or $A \equiv[z, B] C$, then $Q B \in \Lambda$ by Th. 1.7 or by Th. 1.8 , so by induction $Q \tau \in \Lambda$. 
Theorem 1.10. If $A \in \Lambda$ and $B \in A$, then $A \mid B \in \Lambda$.

Proof. Induction on $|A|$. If $A \equiv \tau$ then the proof is trivial. Let $A \equiv\left[x_{1}, A_{1}\right] \ldots\left[x_{k}, A_{k}\right] P s$, where $P \notin[z, E] P^{\prime}$.

(1) If $B \equiv\left[x_{j}, A_{j}\right] \ldots\left[x_{k}, A_{k}\right] P s$ or $B \equiv P s$, then $A \mid B \equiv A \in \Lambda$.

(2) If $B \subset A_{i}$, then $A \mid B \equiv\left[x_{1}, A_{1}\right] \ldots\left[x_{i-1}, A_{i-1}\right]\left(A_{i} \mid B\right) \equiv$ $\left(\left[x_{1}, A_{1}\right] \ldots\left[x_{i-1}, A_{i-1}\right] A_{i}\right) \mid B$ and $\left[x_{1}, A_{1}\right] \ldots\left[x_{i-1}, A_{i-1}\right] A_{i} \in \Lambda$ by $T h, 1.8$, so by induction $A \mid B \in A$.

(3) Let $B \subset P s, B \neq P s$. If $P \equiv \emptyset$ then $B \equiv s$ and $A \mid B \equiv A \in A$. So assume $P \equiv\{K\} P^{\prime}$. Distinguish the cases $B \subset K$ and $B \subset \mathrm{P}^{\prime} \mathrm{s}$. In both cases we may conclude $A \mid B \in \Lambda$ by a similar reasoning as in (2).

Corollary 1.11. If $A \in \Lambda$ and $x \in A$, then $A \mid x \in \Lambda$.

Theorem 1.12. If $\mathrm{QA}$ and $\mathrm{QB} \in \Lambda, \mathrm{Q}[\mathrm{x}, \mathrm{A}] \mathrm{B} \in \Delta$, then $\mathrm{Q}[\mathrm{x}, \mathrm{A}] \mathrm{B} \in \Lambda$. Proof. Induction on $|B|$. Let $B \equiv\left[y_{1}, B_{1}\right] \ldots\left[y_{k}, B_{k}\right]$ Ps, where $P \neq[z, E] P^{\prime}$.

case 1. $\mathrm{P} \equiv \emptyset, \mathrm{k}=0$. Then $\mathrm{Q}[\mathrm{x}, \mathrm{A}] \mathrm{B} \in \Lambda$ by Def. 1.3 (2) or (3). case 2. $P \equiv \emptyset, k \geq 1 . \operatorname{Ca11}\left[y_{1}, B_{1}\right] \ldots\left[y_{k-1}, B_{k-1}\right] \equiv Q^{\prime}$.

(1) Assume $s \equiv y_{k}$. Then $Q^{\dagger} B_{k} \in \Lambda$ by Th. 1.8 , and $Q[x, A] Q^{\prime} B_{k} \in \Delta$ (Th. II.3.8 and Th. II.3.9), so by induction $Q[x, A] Q^{\prime} B_{k} \in \Lambda$, hence $Q[x, A] B \in \Lambda$.

(2) Assume $s \not \equiv y_{k}$. Then $Q Q^{\prime} B_{k}$ and $Q Q^{\prime} s \in \Lambda$ (by the unique $\Lambda$-constructibility), $Q[x, A] Q^{\prime} B_{k}$ and $Q[x, A] Q^{\prime} s \in . \Delta$ (Th. II.3.8 and $T h$. IT.3.9), so by induction $Q[x, A] Q^{\prime} B_{k}$ and $Q[x, A] Q^{\prime} s \in A$. It follows that $\mathrm{Q}[\mathrm{x}, \mathrm{A}] \mathrm{B} \in \Lambda$.

cae 3. $\mathrm{P} \equiv\{E\} \mathrm{P}^{\prime} \cdot \operatorname{Cal1}\left[\mathrm{y}_{1}, \mathrm{~B}_{1}\right] \ldots\left[y_{\mathrm{k}}, \mathrm{B}_{\mathrm{k}}\right] \equiv \mathrm{Q}^{\prime \prime}$. Then $Q Q^{\prime \prime} \mathrm{E}$ and $Q Q^{\prime \prime} P^{\prime} s \in \Lambda$ by Th. 1.7, Typ ${ }^{\star} Q^{\prime \prime} P^{\prime} s \equiv Q Q^{\prime \prime} F^{\prime} \geq Q Q^{\prime \prime}[z, K] L$ and Typ $Q Q^{\prime \prime E} \equiv Q Q^{\prime \prime} E^{\prime} \geq Q Q^{\prime \prime K}$. It follows from Th. II.7.33,Th. II.8.9 and Th. II.8.17 that Typ ${ }^{\star} Q[x, A] Q^{\prime \prime} \mathrm{P}^{\prime} \mathrm{s} \equiv \mathrm{Q}[\mathrm{x}, \mathrm{A}] \mathrm{Q}^{\prime \prime} \mathrm{F}^{\prime} \geq \mathrm{Q}[\mathrm{x}, \mathrm{A}] \mathrm{Q}^{\prime \prime}[\mathrm{z}, \mathrm{K}] \mathrm{L}$ and Typ $Q[x, A] Q^{\prime \prime} E \equiv Q[x, A] Q^{\prime \prime} E^{\prime} \geq Q[x, A] Q^{\prime \prime} K$. By Th. II.3.8 and Th. II. 3.9 $Q[x, A] Q^{\prime \prime} P$ 's and $Q[x, A] Q^{\prime \prime} E \epsilon \Delta$, so by induction they also belong to 
$\Lambda$, hence $Q[x, A] B \in \Lambda$.

Theorem 1.13. If $\mathrm{Q}[\mathrm{x}, \mathrm{A}] \mathrm{B} \in \Lambda$ and $\mathrm{QB} \in \Delta$, then $\mathrm{QB} \in \Lambda$.

Proof. Induction on $|B|$. The proof is similar to the proof of Th. 1.12, with the use of Th. II.3.10 instead of Th. II.3.11.

We shall use the following theorem as a lenma for the important Th. 1.15.

Theorem 1.14. Let $\mathrm{PP}^{\prime} \mathrm{K}, \mathrm{PL} \in \Lambda, \mathrm{PP}^{\prime} \mathrm{L} \in \Delta$ and $\mathrm{TyP}^{*} \mathrm{PP}^{\prime} \mathrm{K} \geq{ }_{\alpha} \mathrm{TyP}^{*} \mathrm{PP} \mathrm{P}^{\prime} \mathrm{L}$. Then PP'L $\in \Lambda$.

Proof. Induction on $\left\|P^{\prime}\right\|$. If $\mathrm{PP}^{\prime} \equiv \emptyset$, the proof is trivial.

case 1. Assume $P \equiv Q\{E\} P^{\prime \prime}$. Then $Q P^{\prime} P^{\prime} K \in \Lambda, Q E \in \Lambda$, TyP* $Q P^{\prime \prime} P^{\prime} K \geq$ $\mathrm{Q}[y, M] N$ and Typ $Q E \geq Q M$. Also: QP"L $\epsilon \Lambda$ and $Q P^{\prime \prime} P ' L \epsilon \Delta$. We now prove that TyP* $Q P^{\prime \prime} P^{\prime} K \geq_{\alpha}$ TyP ${ }^{*} Q P^{\prime \prime} P^{\prime} L$. Let TyP* $P P^{\prime} K \equiv P P^{\prime} K^{\prime}$ and TyP* $P P^{\prime} L \equiv P P^{\prime} L^{\prime}$, then by hypothesis $Q\{E\} P^{\prime \prime} P^{\prime} K^{\prime} \equiv P P^{\prime} K^{\prime} \geq_{\alpha} P P^{\prime} L^{\prime} \equiv$ $Q\{E\} P^{\prime \prime} P^{\prime} L^{\prime}$, so also $Q P^{\prime \prime} P^{\prime} K^{\prime} \geq{ }_{\alpha} Q P^{\prime \prime} P^{\prime} L^{\prime}$ (Th. II.4.6). But TyP ${ }^{*} Q P^{\prime \prime} P^{\prime} K \equiv Q P^{\prime \prime} P^{\prime} K^{\prime}$ and TyP ${ }^{*} Q P^{\prime \prime} P^{\prime} L \equiv Q P^{\prime \prime} P^{\prime} L^{\prime}$ by Th. II.8.6 and Th. II.8.18. It follows by induction that $Q P^{\prime} P ' L \in \Lambda$. Also TyP $^{*} Q P^{\prime \prime} P^{\prime} L \geq Q[y, M] N$, so $Q\{E\} P^{\prime \prime} P^{\prime} L \equiv P P^{\prime} L \in \Lambda$.

case 2. Assume $P \equiv Q$ and $P^{\prime} \equiv Q^{\prime}\{E\} P^{\prime \prime}$. Then $Q Q^{\prime} P^{\prime \prime} K \in \Lambda, Q Q^{\prime} E \in \Lambda$, Typ ${ }^{*} Q Q^{\prime} P^{\prime \prime} K \geq Q Q^{\prime}[y, M] N$ and Typ $Q Q^{\prime} E \geq Q Q^{\prime} M$. Also: $Q Q^{\prime} P^{\prime \prime} L \epsilon \Delta$ and Typ * $Q Q^{\prime} P^{\prime \prime} K \geq{ }_{\alpha}$ Typ* $Q Q^{\prime} P^{\prime \prime} L$ (which can be proved as in case 1 ), so by induction $Q Q^{\prime} P^{\prime \prime} L \in \Lambda$. Since Typ ${ }^{*} Q Q^{\prime} P^{\prime \prime} L \geq Q Q^{\prime}[y, M] N$ it follows that $Q Q^{\prime}\{E\} P^{\prime \prime} L \equiv P P^{\prime} L \in \Lambda$.

case 3. Assume $P \equiv Q$ and $P^{\prime} \equiv Q^{\prime}$. If $Q^{\prime} \equiv \emptyset$ there is nothing to prove. Let $Q^{\prime} \equiv\left[x_{1}, M_{1}\right] \ldots\left[x_{n}, M_{n}\right]$ for $n \geq 1$. Since $Q L$ and $Q Q^{\prime} L \in \Delta, x_{i}$ cannot occur in $Q L$ (Th. II.3.8) or in $Q\left[x_{1}, M_{1}\right] \ldots$ $\left[x_{i-1}, M_{i-1}\right] M_{i}$. It follows from $Q Q^{\prime} K \in \Lambda$ (Th. 1.8) that $\mathrm{QM}, Q\left[\mathrm{x}_{1}, \mathrm{M}_{1}\right] \mathrm{M}_{2}, \ldots, \mathrm{Q}\left[\mathrm{x}_{1}, \mathrm{M}_{1}\right] \ldots\left[\mathrm{x}_{\mathrm{n}-1}, \mathrm{M}_{\mathrm{n}-1}\right] \mathrm{M}_{\mathrm{n}} \in \Lambda$. So also $Q\left[x_{1}, M_{1}\right] L, Q\left[x_{1}, M_{1}\right]\left[x_{2}, M_{2}\right] L, \ldots, Q Q ' L \in \Lambda$ by Th. II.3.11 and Th. 1,12 . 
Theorem 1.15. If $A \in \Lambda$, then $\operatorname{Typ}^{n} A \in \Lambda$ for all permissible $n$.

Proof. Let $A \equiv P_{1}[x, B] P_{2} x$, then Typ $A \equiv P_{1}[x, B] P_{2} F r B$. Since $A \in \Lambda$ : $\mathrm{P}_{1} \mathrm{~B} \in \Lambda$ (Th. 1.8), so $\mathrm{P}_{1} \mathrm{FrB} \in \Lambda$ (Th. 1.6). Also Typ $A \in \Delta$ (Th. II.8.2) and Typ ${ }^{*} A \geq_{\alpha} \operatorname{Typ}^{*}$ (Typ A). Now, applying Th. 1.14, we obtain Typ $A \in \Lambda$. The theorem follows directly.

\section{§ 2. THE NORMALIZATION THEOREM}

In this section we shall prove the normalization theorem: if $A \in A$, there is a $B$ in normal form such that $A \geq B$ (B is said to be in normal form if there are no reductions $B_{B}^{\prime} B^{\prime}$ or $B_{\eta}^{\prime} B^{\prime}$ ). We do this by the aid of a norm $\rho$, which is a partial function from expressions in $\Delta$ to expressions in $\Delta$, and which has the following powerful properties with relation to $\Lambda$ :

(1) If $A \in A$, then $\rho(A)$ is defined.

(2) If $A \in \Lambda$ and $A \geq B$, then $\rho(A) \geq_{\alpha} \rho(B)$.

(3) If $A \in \Lambda$ and $\operatorname{Deg} A>1$, then $\rho(A) \geq_{\alpha} \rho(\operatorname{Typ}(A))$.

Hence this norm is invariant (apart from $\alpha$-reduction) with respect to reduction and typing.

We first define $\rho_{A}$ for every $A \in \Delta$. This $\rho_{A}$ is a partial function from subexpressions of $A$ to expressions. It is rather in contradiction to our philosophy to define the norm with respect to subexpressions, which need not belong to $\Delta$. We could have avoided this by giving a definition of the norm in the line of our second definition of $\Delta$, only considering norms of expressions in $\Delta$. This, however, would have impaired understanding of the following and would have led to laborious descriptions. On the other hand, in this section the context of a subexpression will always be clear, so that no confusion can arise.

In the following inductive definition of $\rho_{A}$ we do not explicitly indicate which occurrence of a subexpression in an expression is meant, since this will be clear from the context. 
Definition 2.1. Let $A \in \Delta$.

(1) If $\tau \subset A$ then $\rho_{A}(\tau) \equiv \tau$.

(2) If $x \in A, A \mid x \equiv Q_{1}[x, B] Q_{2} x$ and if $\rho_{A}(B)$ is defined, then $\rho_{A}(x) \equiv \rho_{A}(B)$.

(3) If $[x, B] C \subset A$, and if both $\rho_{A}(B)$ and $\rho_{A}(C)$ are defined, then $\rho_{A}([x, B] C) \equiv\left[x, \rho_{A}(B)\right] \rho_{A}(C)$.

(4) If $\{B\} C \subset A$, if both $\rho_{A}(B)$ and $\rho_{A}(C)$ are defined and $\rho_{A}(C) \equiv$ $[y, D] E$ where $D \geq_{\alpha} \rho_{A} B$, then $\rho_{A}(\{B\} C) \equiv E$.

From this definition it can easily be seen that, if $\rho_{A} A$ is defined for $A \in \Delta, \rho_{A} A$ contains no bound variables.

The following theorem is obvious:

Theorem 2.2. If $A \in \Delta$ and if $\rho_{A}(A)$ is defined, then $\rho_{A}(A) \in \Delta$; if, moreover, $A \geq_{\alpha} B$, then $\rho_{A}(A) \geq_{\alpha} \rho_{B}(B)$ and $\left|\rho_{A}(A)\right|=\left|\rho_{B}(B)\right|$.

The binding variables in $\rho_{A} A$ will be irrelevant to our purposes. We might as well do without them. Our reason for retaining them is personal taste: we find the property $\rho_{A}(A) \in \Delta$ agreeable.

In trying to calculate $\rho_{A}(A)$ for a certain $A \in \Delta$, we apply the four rules of Def. 2.1; the only event in which this calculation can break down prematurely (before $\rho_{A}(A)$ is obtained), is when we encounter a subexpression $\{B\} C \subset A$ for which the conditions stated in Def. 2.1 (4) are not fulfilled.

These conditions may be considered as a weaker form of the applicability condition (cf. Section I.6, where this is explained in an informal manner): (1) $C$ must have a norm with a functional character: $\rho_{A} C \equiv[y, D] E$, and (2) $B$ has a norm which behaves as an appropriate argument for the "function" $\rho_{A} C: \rho_{A} B \geq_{G} D$. If these conditions are fulfilled, the norm of $\{B\} C$ is defined as the result of the application of the "function" $\rho_{A} C$ to the "argument" $\rho_{A} B$ : $\rho_{A}(\{B\} C) \equiv E$; if these conditions are not fulfilled, the norm of $\{B\} C$ is not defined, and neither is the norm of $A$. 
Note that the norm of a bound variable is defined as the norm of its "type": if $[\mathrm{x}, \mathrm{B}]$ is the binding abstractor of $x$, then $\rho_{A}(x) \equiv \rho_{A}(B)$.

The existence of $\rho_{A} A$ for a certain $A \in \Delta$ indicates that some weak functional condition is fulfilled. Surprisingly enough, the existence of $\rho_{A} A$ already guarantees that there is a normal form for A. We shall prove this in Th. 2.17. We are especially interested in. normalization properties of expressions in $\Lambda$. We note that expressions in $\Lambda$ have, so to say, a much stronger functional character than is required for the existence of the norm of expressions. Th. 2.7, stating that $\rho_{A} A$ exists for $A \in \Lambda$, is not hard to prove.

If in the following we speak of the norm of a subexpression $B$ of a certain expression $A$, it will be clear which $A$ we mean, even if we do not state this explicitly. In such cases we shall write $\rho(B)$ instead of $\rho_{A}(B)$.

If $A \in \Delta, B \subset A$ and $\rho_{A}(B)$ is defined, we cal1 $B \rho_{A}$-normable. Here, too, we speak of "p-normable $B$ " if it is clear which $A$ (with $B \subset A \in \Delta)$ we mean. If $Q \tau \in \Delta$, if $Q \tau$ is $p$-normable and $\rho(Q \tau) \equiv Q^{\prime} \tau$, we cal1 $Q \rho$-normable, and we abbreviate $\rho(Q) \equiv Q^{\prime}$.

Theorem 2.3. If $A \epsilon \Delta, A$ is $p$-normable and $B \subset A$, then $B$ is $p$-normable.

Proof. Induction on $|A|$, with the use of the definition of $B \subset A$ (Def. II.2.5).

Theorem 2.4. If $Q A \in \Delta$ and $Q A$ is $p$-normable, then $Q$ and $A$ are $p-$ normable and $\rho(Q A) \equiv(\rho Q) \rho A$; if $Q A \in \Delta$, and if $Q$ and $A$ are $\rho$-normable, then $Q A$ is $p$-normable and $\rho(Q A) \equiv(\rho Q) \rho A$.

Proof. Induction on $\|Q\|$.

Theorem 2.5. If $A \in \Delta$, if $A$ is $p$-normable and $A \geq B$, then $B$ is $p^{-}$ normable and $\rho A \geq_{\alpha} \rho B$. 
Proof. First assume that $A \geq^{\prime} B$. We proceed by induction on the length of proof that $A \geq^{\prime} B$. If $A \geq_{\alpha}^{\prime} B$ then the proof is trivial; this case is expressed in Th. 2.2.

Ia. $A \geq^{\prime} B$ is $Q\{C\}[x, D] E \geq_{B}^{\prime} Q(x:=C) E$. Since $A$ is $\rho$-normable: $\rho([x, D] E) \equiv[x, \rho D] \rho E$ and $\rho C \geq_{\alpha} \rho D \equiv \rho x$ (see Th. 2.3 and Th. 2.4). Moreover, $\rho \mathrm{A} \equiv \rho(Q\{C\}[\mathrm{x}, D] E) \equiv(\rho Q) \rho E$. We now prove for this $\mathrm{x}, \mathrm{C}$ and $\mathrm{E}$ :

Lemma. If $K \subset E$, then $(x:=C) K$ is $\rho$-normable and $\rho(x:=C) K{ }_{\alpha} \rho K$. Proof of the lemma. Induction on $|\mathrm{K}|$.

(1a) If $K \equiv x$, then $(x:=C) K \equiv F r C$ and $\rho(x:=C) K \equiv \rho F r C \geq_{\alpha} \rho C \geq_{\alpha}$ $\rho x \equiv \rho K$.

(1b) If $\mathrm{K} \equiv \mathrm{y} \equiv \mathrm{x}$ or $\mathrm{K} \equiv \tau$, then $(\mathrm{x}:=\mathrm{C}) \mathrm{K} \equiv \mathrm{K}$ and $\rho(\mathrm{x}:=\mathrm{C}) \mathrm{K} \equiv \rho \mathrm{K}$. (2) If $\mathrm{K} \equiv[\mathrm{y}, \mathrm{F}] \mathrm{G}$, then $\rho \mathrm{K} \equiv[\mathrm{y}, \rho \mathrm{F}] \rho \mathrm{G}$. Note that $\mathrm{y} \equiv \mathrm{x}$. By induction: $(x:=C) F$ and $(x:=C) G$ are $\rho$-normable, $\rho(x:=C) F \geq_{\alpha} \rho F$ and $\rho(x:=C) G \geq_{\alpha} \rho G$. So $(x:=C) K$ is $\rho$-normable and $\rho(x:=C) K \equiv$ $[y, \rho(x:=C) F] \rho(x:=C) G \geq{ }_{\alpha}[y, \rho F] \rho G \equiv \rho K$.

(3) If $K \equiv\{F\} G$, then $\rho G \equiv[y, L] H$ and $L \geq_{\alpha} \rho F$, and $\rho K \equiv H$. By induction: $(x:=C) F$ and $(x:=C) G$ are $\rho$-normable, $\rho(x:=C) F \geq_{\alpha} \rho F$ and $\rho(x:=C) G \geq_{\alpha} \rho G$. It follows that $\rho(x:=C) G \equiv\left[z, L^{\prime}\right] H^{\prime}$ and $L^{\prime} \geq_{\alpha} L \geq_{\alpha} \rho F \geq_{\alpha} \rho(x:=C) F$, so $(x:=C) K$ is $\rho$-normable and $\rho(x:=C) K \equiv H^{\prime} \geq_{\alpha} H \equiv \rho K$.

It follows that $B$ is $\rho$-normable (since $E \subset E$ ), and $\rho B \equiv(\rho Q) \rho(x:=C) E \geq_{\alpha}(\rho Q) \rho E \equiv \rho A$.

Ib. $A \geq B$ is $Q[x, C]\{x\} D \geq \geq_{n}^{\prime} Q D$. Since $A$ is $\rho$-normable: $Q C$ is $\rho$-normable, $\rho x \equiv \rho C, \rho D \equiv[y, L] H$ and $L \geq_{\alpha} \rho x \equiv \rho C$, so $\rho A \equiv(\rho Q)[x, \rho C] H \geq_{\alpha}$ $(\rho Q)[y, \rho C] H \geq_{\alpha}(\rho Q) \rho D \equiv \rho Q D \equiv \rho B$.

II. $A \geq^{\prime} B$ is a direct consequence of a monotony rule. It depends on the monotony rule which of the following three cases applies:

IIa. $A \geq^{\prime} B$ is $Q\{C\} E \geq^{\prime} Q\{C\} F$ as a direct consequence of $Q E \geq^{\prime} Q F$. Since $A$ is $p$-normable: $Q E$ is $\rho$-normable. By induction: $Q F$ is $\rho-$ normable, and $\rho Q E \equiv(\rho Q) \rho E \geq_{\alpha}(\rho Q) \rho F \equiv \rho Q F$, so $\rho E \geq_{\alpha} \rho F$. Moreover, 
$\rho E \equiv[y, L] H$ and $\rho C \geq_{\alpha} L$, so also $\rho F \equiv\left[z, L^{\prime}\right] H^{\prime}$, where $L^{\prime} \geq_{\alpha} L$ and $\mathrm{H}^{\prime} \geq_{\alpha} \mathrm{H}$. It follows that $\{\mathrm{C}\} \mathrm{F}$ is $\rho$-normable, and $\rho\{C\} F \equiv \mathrm{H}^{\prime} \geq_{\alpha} H$. So $\rho B \geq_{\alpha}(\rho Q) \rho H \equiv \rho A$.

IIb. $A z^{\prime} B$ is $Q[x, C] E \geq^{\prime} Q[x, D] E$ as a direct consequence of $Q C \geq \geq^{\prime} Q D$.

Then $\rho A \equiv(\rho Q)[x, \rho C] \rho E$. By induction: $Q D$ is $\rho$-normable and $\rho Q C \equiv$ $(\rho Q) \rho C \geq_{\alpha}(\rho Q) \rho D \equiv \rho Q D$, so $\rho C \geq_{\alpha} \rho D$. Hence $B$ is $\rho$-normable and $\rho A \geq_{\alpha}(\rho Q)[x, \rho D] \rho E \equiv \rho B$.

IIc. $A \geq^{\prime} B$ is $Q\{C\} E \geq^{\prime} Q\{D\} E$ as a direct consequence of $Q C \geq^{\prime} Q D$. Then $\rho E \equiv[x, L] H$ and $L \geq_{\alpha} \rho C$. By induction: $Q D$ is $\rho$-normable and $\rho Q C \equiv(\rho Q) \rho C \geq_{\alpha}(\rho Q) \rho D \equiv \rho Q D$, so $\rho C \geq_{\alpha} \rho D$. Hence $B$ is $\rho$-normable and $\rho B \equiv(\rho Q) H \equiv \rho A$.

Finally, if $A \geq B$ is a multiple-step reduction, decompose the reduction and apply the above.

Theorem 2.6. If $A \in \Delta$, Deg $A>1$ and $A$ is $p$-normable, then Typ $A$ is $\rho$-normable and $\rho A \geq{ }_{\alpha} \rho$ Typ $A$.

Proof. Let $A \equiv P_{1}[x, B] P_{2} x$, then Typ $A \equiv P_{1}[x, B] P_{2} F r B$. It is not hard to show that $\rho \mathrm{Fr} B \geq_{\alpha} \rho B \equiv \rho x$. Let $\mathrm{P}_{1}[\mathrm{X}, \mathrm{B}] \mathrm{P}_{2} \equiv \mathrm{P}^{\prime} \mathrm{P}^{\prime \prime}$. Next prove by induction on $\left\|P^{\prime \prime}\right\|$ that $P^{\prime \prime} F r B$ is $\rho$-normable, and $\rho\left(P^{\prime \prime} F r B\right) \geq_{\alpha} \rho\left(P^{\prime \prime} x\right)$.

Theorem 2.7. If $A \in \Lambda$, then $A$ is $p$-normable (i.e. $p$ is a total function on $\Lambda$ ).

Proof. Induction on the length of proof of $A \in \Lambda$.

(1) $A \equiv \tau$ : trivial.

(2) $\mathrm{A} \equiv \mathrm{Q}[\mathrm{X}, \mathrm{B}] \mathrm{x}$ or $\mathrm{Q}[\mathrm{x}, \mathrm{B}] \tau \in \Lambda$ as a direct consequence of $\mathrm{QB} \in \Lambda$. Then by induction $Q B$ is $\rho$-normable, hence $Q$ is $\rho$-normable and $\rho B \equiv \rho x$. Hence $A$ is $\rho$-normable.

(3) $A \equiv Q[x, B] y \in \Lambda$ as a direct consequence of $Q B \in \Lambda$ and $Q y \in \Lambda$. By induction: $Q B$ and $Q y$ are $\rho$-normable, hence $Q, B$ and $y$ are $\rho-$ normable, so $\mathrm{A}$ is $\rho$-normable. 
(4) $A \equiv Q\{B\} C \in \Lambda$ as a direct consequence of $Q B \in \Lambda, Q C \in \Lambda$, and the Q-applicability of $Q C$ to $Q B$. Then $Q B$ and $Q C$ are $p$-normable (induction), and so are $Q, B$ and $C$. The Q-applicability implies that Typ ${ }^{*} \mathrm{QC} \geq \mathrm{Q}[\mathrm{x}, \mathrm{K}] \mathrm{L}$ and Typ $\mathrm{QB} \geq \mathrm{QK}$. From Th. 2.5 and $\mathrm{Th}$. 2.6: Typ ${ }^{*} \mathrm{QC}$ and $\mathrm{Q}[\mathrm{x}, \mathrm{K}] \mathrm{L}$ are $\rho$-normable, $\rho \mathrm{QC} \geq_{\alpha} \rho$ Typ ${ }^{*} \mathrm{QC} \geq_{\alpha}$ $(\rho Q)[x, \rho K] \rho L$ (so $\rho C \geq_{\alpha}[x, \rho K] \rho L$ ) and $\rho Q B \geq_{\alpha} \rho$ Typ $Q B \geq_{\alpha} \rho Q K$ (so $\left.\rho B \geq_{Q} \rho K\right)$. Hence $\{B\} C$ is $\rho$-normable and so is $A$.

Instead of: $\rho$ is total on $\Lambda$, we also say: $\Lambda$ is $\rho$-normable.

From Th. 2.5 we derive:

Theorem 2.8. If $A, B \in \Lambda$ and $A \sim B$, then $\rho A \geq{ }_{\alpha} \rho B$.

We sha11 now prove the normalization theorem for $p$-normable expressions.

Definition 2.9. A $\in \Delta$ is normal (or in normal form) if there are no reductions $A z_{B}^{\prime} B$ or $A z_{\eta}^{\prime} B$; $A$ is normalizable (or $A$ has a normal form) if there exists a normal $C$ such that $A \geq C$ ( $C$ is called a normat form of $\mathrm{A}$ ).

Definition 2.10. $A \in \Delta$ is $B$-normal if there is no reduction $A Z_{B}^{\prime} B$; $A$ is $\beta$-normalizable (or $A$ has a $B$-normal form) if there exists a $B$ normal $C$ such that $A \geq_{B} C$ ( $C$ is called a $B$-normal form of $A$ ).

Hence $A$ is normal if $A$ admits of neither $B-$, nor $n$-reductions; $A$ is $\beta$-normal is $A$ admits of no $\beta$-reductions (except trivial ones).

Theorem 2.11. If $A \in \Delta$ is normal and $A \geq_{\alpha} B$, then $B$ is normal. If $A \in \Delta$ is $B$-normal and $A \geq_{\alpha} B$ or $A \geq_{\eta} B$, then $B$ is $B$-normal.

Proof. The only non-trivial statement is that $B$ is $B$-normal if $A$ is $B$-normal and $A \geq_{\eta} B$. It can, however, easily be seen that a singlestep n-reduction of a $\beta$-normal expression cannot introduce the possibility of a single-step $\beta$-reduction. 
We restate the following well-known theorem:

Theorem 2.12. If $A \in \Delta$ is $\beta$-normalizable, then $A$ is normalizable. Proof. As a result of Th. 2.11, n-reductions of $A$ do not cancel the $B$-normal character. But the possible number of single-step $n$-reductions applicable to $A$ is finite, since the expression becomes shorter with each step.

Theorem 2.13. If $A \in \Delta$ is $\beta$-normalizable, the $\beta$-normal form of $A$ is unique but for $\alpha$-reductions.

Proof. Let $C$ and $D$ be $\beta$-normal, $A \geq_{B} C$ and $A \geq_{B} D$. Then, by the Church-Rosser theorem for $\beta$-reduction (Th. II.6.43): there is an $E$ such that $C \geq_{\beta} E$ and $D \geq_{\alpha, \beta} E$. Hence $C \geq_{\alpha} D$.

Theorem 2.14. Assume that $Q\left\{A_{k}\right\} \ldots\left\{A_{1}\right\} B$ is in $\Delta$ and $\rho$-normable. Then $|\rho B|>\sum_{i=1}^{k}\left|\rho A_{i}\right|$.

Proof. Induction on $k$. If $k=0$ the proof is trivial. Let $k>0$. Then $\left\{A_{1}\right\} B$ is $\rho$-normable, hence $\rho B \equiv[x, M] N$ and $\rho A_{1} \geq_{\alpha} M$, so $\left|\rho A_{1}\right|=|M|$. Moreover, $\rho\left\{A_{1}\right\} B \equiv N$, hence $|N|>\sum_{i=2}^{k}\left|\rho A_{i}\right|$ by induction. It follows that

$$
|\rho B|=|[x, M] N|>|M|+\sum_{i=2}^{k}\left|\rho A_{i}\right|=\sum_{i=1}^{k}\left|\rho A_{i}\right| .
$$

Definition 2.15. Assume that $A$ is in $\Delta$ and $\rho$-normable, $A \equiv Q\left\{C_{n}\right\} \ldots$ .. $\left\{C_{1}\right\} F$ for some $n \geq 1$ and $F \not\{M\} N$. Then $\sigma(A)=\sum_{i=1}^{n}\left|\rho C_{i}\right|$. If $A \equiv Q s$ (with $s \equiv x$ or $s \equiv \tau)$, then $\sigma(A)=0$.

Theorem 2.16. Assume that $A$ is in $\Delta$ and $\rho$-normable, $A \equiv Q\left\{C_{n}\right\} \ldots$ $\left\{C_{1}\right\} F, F \neq\{M\} N$, and let $Q C_{i}$ (for $1 \leq i \leq n$ ) and $Q F$ be $\beta$-normal. Then $A$ is $\beta$-normalizable. 
Proof. Induction on $\sigma(A)$.

(1) If $\sigma(A)=0$, then $n=0$ and $A=Q F$ in $B$-normal form.

(2) Let $\sigma(A)>0$. A is $B$-normal if $n=0$. Let $n \geq 1$. We proceed by induction on $|F|$. If $F \equiv y$, then $A$ is in $\beta$-normal form ( $F \equiv \tau$ cannot occur since $A$ is $p$-normable). So let $|F|>1$. Then $F \equiv[X, D] E$.

(i) Assume that $E \equiv\left\{\mathrm{H}_{\mathrm{m}}\right\} \ldots\left\{\mathrm{H}_{1}\right\} y$ where $\mathrm{y} \equiv \mathrm{x}$ and $\mathrm{m} \geq 0$. Then $A \geq Q\left\{C_{n}\right\} \ldots\left\{C_{2}\right\}\left\{\left(x:=C_{1}\right) H_{m}\right\} \ldots\left\{\left(x:=C_{1}\right) H_{1}\right\} y$. Now note that $Q\left\{C_{1}\right\}[x, D] H_{i} \in \Delta, \rho-n o r m a b l e$, and $\sigma\left(Q\left\{C_{1}\right\}[x, D] H_{i}\right)=\left|\rho C_{1}\right| \leq \sigma(A)$. Moreover, $\left|H_{i}\right|<|E|$, so by induction $Q\left\{C_{1}\right\}[X, D] H_{i}$ is $\beta$-normalizable. Since $Q C_{i}, Q D$ and $Q H_{i}$ are $\beta$-normal, the $\beta$-normalization of $Q\left\{C_{1}\right\}[x, D] H_{i}$ must commence with $Q\left\{C_{1}\right\}[x, D] H_{i} \geq_{B}^{\prime} Q\left(x:=C_{1}\right) H_{i}$, so $Q\left(x:=C_{1}\right) H_{i}$ is $\beta$-normalizable; say $Q\left(x:=C_{1}\right) H_{i} \geq Q K_{i}$ in $\beta$-normal form. It follows that $A \geq Q\left\{C_{n}\right\} \ldots\left\{C_{2}\right\}\left\{K_{m}\right\} \ldots\left\{K_{1}\right\} y$ in $B$-normal form.

(ii) Assume that $\mathrm{E} \equiv\left\{\mathrm{H}_{\mathrm{m}}\right\} \ldots\left\{\mathrm{H}_{1}\right\}_{\mathrm{T}}$ where $\mathrm{m} \geq 0$. Again, analogous$1 y$ to (i), $A$ is $B$-normalizable. (Moreover, $m$ must be 0 since $A$ is p-normable.)

(iii) Assume that $E \equiv\left\{\mathrm{H}_{\mathrm{m}}\right\} \ldots\left\{\mathrm{H}_{1}\right\} \mathrm{x}$ where $\mathrm{m} \geq 0$. Then $\mathrm{A} \geq \mathrm{A}^{\prime} \equiv$ $Q\left\{C_{n}\right\} \ldots\left\{C_{2}\right\}\left\{K_{m}\right\} \ldots\left\{K_{1}\right\} F r C C_{1}$, where we obtain the $\beta$-normal $Q K_{i}$ as in (i). If now $\mathrm{FrC}_{1}$ is a variable or if $\mathrm{FrC}_{1}$ begins with an applicator, we have obtained a $B$-normal form. If $\operatorname{FrC}_{1} \equiv[y, M] N$, then $\sigma\left(A^{\prime}\right)=\sum_{i=2}^{n}\left|\rho c_{i}\right|+\sum_{j=1}^{m}\left|\rho K_{i}\right|<\left|\rho F r C_{1}\right|$ (by Th. 2.14 and Th. 2.5) $=$ $\left|\rho c_{1}\right| \leq \sigma(A)$, so by the induction hypothesis: $A^{\prime}$ is $B$-normalizable, so $A$ is $\beta$-normalizable too, or $n=1$ and $m=0$, whence $A^{\prime}$ is $\beta$-normal.

(iv) Assume that $\mathrm{E} \equiv\left[\mathrm{y}, \mathrm{H}_{1}\right] \mathrm{H}_{2}$. Then

$A \geq Q\left\{C_{n}\right\} \ldots\left\{C_{2}\right\}\left[y,\left(x:=C_{1}\right) H_{1}\right]\left(x:=C_{1}\right) H_{2} \geq Q\left\{C_{n}\right\} \ldots\left\{C_{2}\right\}\left[y, K_{1}\right] K_{2}$, where we again obtain the $\beta-n o r m a l ~ Q\left[y, K_{1}\right] \mathrm{K}_{2}$ as in (i). Since $\sigma\left(Q\left\{C_{n}\right\} \ldots\left\{C_{2}\right\}\left[y, K_{1}\right] K_{2}\right)=\sum_{i=2}^{n}\left|\rho C_{i}\right|<\sigma(A)$, it follows by induction that $A$ is $\beta$-normalizable, or $n=1$ and $Q\left[y, K_{1}\right] K_{2}$ is $\beta$-normal. 
Theorem 2.17 ( $\beta$-normalization theorem). If $A \in \Delta$ is $p$-normable, then $A$ is $\beta$-normalizable.

Proof. Induction on the length of proof of $A \in \Delta$.

(1) $\mathrm{A} \equiv \tau$; trivial.

(2) $A \equiv Q[x, B] x$ or $A \equiv Q[x, B] \tau \in \Delta$ as a direct consequence of $Q B \in \Delta$. Then by induction $Q B$ is $\beta$-normalizable, so $Q B \geq_{B} Q^{\prime} B^{\prime}$ in $B$-normal form, and $A \geq_{B} Q^{\prime}\left[x, B^{\prime}\right] x$ or $A \geq_{\beta} Q^{\prime}\left[x, B^{\prime}\right] \tau$ in $B$-normal form.

(3) $\mathrm{A} \equiv \mathrm{Q}[\mathrm{x}, \mathrm{B}] \mathrm{y} \in \Delta$ as a direct consequence of $\mathrm{QB} \in \Delta$ and $\mathrm{Qy} \in \Delta$. Then by induction $Q B \geq{ }_{B} Q^{\prime} B^{\prime}$ in $B$-normal form, so $A \geq Q^{\prime}\left[x, B^{\prime}\right] y$ in $\beta$-normal form.

(4) $A \equiv Q\{B\} C \in \Delta$ as a direct consequence of $Q B \in \Delta$ and $Q C \in \Delta$. Then by the induction hypothesis: $Q B \geq_{\beta} Q^{\prime} B^{\prime}$ in $\beta$-normal form (with $\|Q\|=\|Q '\|$ ) and $Q C \geq_{B} Q^{\prime \prime} C^{\prime}$ in $B$-normal form (with $\|Q\|=$ $\|Q "\|)$. From $T h .2 .13$ and induction on $\|Q\|$ it follows that $Q^{\prime \prime} C^{\prime} \geq_{\alpha} Q^{\prime} C^{\prime}$. Also $Q\{B\} C \geq_{\beta} Q^{\prime}\left\{B^{\prime}\right\} C^{\prime}$, which is in $\beta$-normal form if $C^{\prime} \equiv[X, D] E$. So all that is left to prove is that $Q^{\prime}\left\{B^{\prime}\right\}[x, D] E$ is $\beta$-normalizable. But this follows from Th. 2.16 and Th. 2.5.

Theorem 2.18 (normalization theorem for $\Lambda$ ). If $A \in \Lambda$ then $A$ is $\beta-$ normalizable and normalizable.

Proof. Follows from Th. 2.17, Th. 2.12 and Th. 2.7.

In fact we proved that $A \in \Lambda$ is effectively normalizable, since all our proofs are constructive, which implies that the normal form of $A \in \Lambda$ is effectively computable. 


\section{3. STRONG NORMALIZATION}

In the previous section we have proved normalization for $\Lambda$. This guarantees that for every $A \in \Lambda$ there is a reduction which leads to a normal form. However, we do not yet know whether an arbitrary sequence of single-step reductions, beginning with $A$, terminates (in a normal form). We shall prove this in this section. The property that an arbitrary sequence of single-step reductions, beginning with some $A$, terminates, will be called the property of strong nomalization.

In the proof we shall use $\beta_{1}$-reduction and $\beta_{2}$-reduction, introduced in Section II.6. A feature of $B_{1}$-reduction is that "scars" of old $B_{1}$-reductions are retained. We shall first prove strong normalization for $\Lambda$ as to $\beta_{1}$-reduction, and derive strong normalization for $\Lambda$ as to $\beta$-reduction; finally, we shall incorporate $n$-reductions.

Definition 3.1. A $\in \Delta$ is $\beta_{1}$-normal (or in $\beta_{1}$-normal form) if there is no $B$ such that $A \geq_{B}^{\prime} B$; $A$ is $B_{1}$-normalizable (or $A$ has a $B_{1}-$ normal form) if there exists a $B_{1}$-normal $C$ such that $A \geq_{B_{1}} C$ (C is then called a $B_{1}$-nomal form of $A$ ). The concepts $B_{2}$-normal, $B_{2}-$ normal form and $\mathrm{B}_{2}$-normalizable are defined analogously.

Theorem 3.2. If $A \in \Delta, A$ is $B_{1}$-normal and $A \geq_{\alpha} B$, then $B$ is $B_{1}-$ normal.

Theorem 3.3. If $A \in \Delta$ is $\beta_{1}$-normalizable, the $\beta_{1}$-normal form is unique but for $\alpha$-reduction.

Proof. This follows from $C R$ for $B_{1}$-reductions (Th. II,6.38).

Theorem 3.4. If $A \in \Delta, A$ is $p$-normable and $A \geq_{B} B$, then $B$ is $p-$ normable and $\rho A \geqslant \alpha \rho B$. 
Proof. First assume that $A \geq_{B}^{\prime} B$. We proceed with induction on the length of proof of $A \geq{ }_{B}^{\prime} B$.

I. Let $A \equiv Q\{C\} \hat{P}[x, D] E \geq_{\beta_{1}} Q\{C\} \hat{P}[\stackrel{\circ}{x}, D](x:=C) E \equiv B$. Then $\hat{P}[x, D] E$ is $p$-normable. It is easy to see (induction on $\|P\|$ ) that $\rho(\hat{P}[x, D] E) \equiv \rho([x, D] E) \equiv[X, \rho D] \rho E$. So $\rho C \geq_{\alpha} \rho D$. As in the lemma occurring in the proof of Th. 2.5 we can prove that $(x:=C) E$ is $p-$ normable and that $\rho E \geq_{\alpha} \rho((x:=C) E)$. It follows that $B$ is $p$-normable and $\rho A \geq_{\alpha} \rho B$.

II. $A \geq_{B}^{\prime} B$ is a direct consequence of a monotony.

In all three cases the proof is identical to that given in part II of the proof of Th. 2.5 .

Finally, if $A \geq_{\beta_{1}} B$ is a multiple-step reduction, decompose the reduction into single-step $\beta_{1}$-reductions and apply the above.

We shall now prove the $\beta_{1}$-normalization theorem. We do this in quite the same manner in which we proved the $\beta$-normalization theorem. In fact, if we had begun by proving the $\beta_{1}$-normalization theorem, the B-normalization theorem would have been a corollary. We have not chosen this order because in the following proof the main lines are obscured by the presence of a number of $\beta$-chains $\hat{p}_{i}$. In contrast to this, the line of thought in the proof of the B-normalization theorem, given in the previous section, is much more lucid.

Definition 3.5. Let $A \in \Delta, A \equiv P_{1} \hat{P B}$ and let $\hat{P}$ be such that, for each $[x, c]$ for which $\hat{P} \equiv P_{2}[x, C] P_{3}$, it holds that $x \notin P_{3} B$. Then we call $\hat{P}$ an ineffective B-chain, and write $A \equiv P_{1} P B$.

Theorem 3.6. If $A \in \Delta$ is $\beta_{1}$-normal and $B \subset A$, then $B$ has the form $B \equiv \stackrel{\circ}{P}_{0}\left[x_{1}, A_{1}\right] \stackrel{\circ}{P}_{1} \ldots\left[x_{n}, A_{n}\right] P_{n}\left\{B_{1}\right\} \mathcal{P}_{1}^{\circ} \ldots\left\{B_{\ell}\right\} P_{\ell}^{\circ} s$, with $s \equiv x_{i}$ or $s \equiv \tau$.

Proof. Induction on $|B|$. 
Theorem 3.7. Let $Q \hat{\mathrm{P}}_{\mathrm{k}+1}\left\{\mathrm{~A}_{k}\right\} \hat{\mathrm{P}}_{\mathrm{k}}\left\{\mathrm{A}_{\mathrm{k}-1}\right\} \hat{\mathrm{P}}_{\mathrm{k}-1} \ldots\left\{\mathrm{A}_{1}\right\} \hat{\mathrm{P}}_{1} \mathrm{~B}$ belong to $\Delta$ and be $\rho$-normable. Then $|\rho B|>\sum_{i=1}^{k}\left|\rho A_{i}\right|$.

Proof. Analogous to the proof of Th. 2.14. Note again that for $p-$ normable $\hat{\mathrm{PC}}$ it holds: $\rho(\hat{\mathrm{P} C}) \equiv \rho \mathrm{C}$.

Definition 3.8. Let $A \in \Delta$ and assume that $A$ is $\rho$-normable. Let $A \equiv Q P_{n+1}\left\{C_{n}\right\} P_{n}^{\circ} \cdots\left\{C_{1}\right\} P_{1} F$, where $F \equiv \tau, F \equiv x$ or $F \equiv[y, M] N$ with $y \in N$. Then $\sigma_{1}(A)=\sum_{i=1}^{n} \rho\left|c_{i}\right|$ if $n \geq 1$, and $\sigma_{1}(A)=0$ in case $n=0$ and $\stackrel{\circ}{\mathrm{P}}_{\mathrm{n}+1} \not \emptyset$. Moreover, $\sigma_{1}(\mathrm{~A})=0$ if $\mathrm{A} \equiv \mathrm{Q} \tau$ or $Q \mathrm{x}$.

Theorem 3.9. Let $A$ belong to $\Delta$ and be $p$-normable, let $A \equiv Q \stackrel{\circ}{P}_{n+1}\left\{C_{n}\right\} \stackrel{\circ}{P}_{n} \ldots\left\{C_{1}\right\} \stackrel{\circ}{P}_{i} F$, where $F \equiv \tau, F \equiv x$ or $F \equiv[x, D] E$ with $x \in E$. Let $Q C_{i}, Q F$ and $Q P_{i} \tau$ be $B_{1}$-normal. Then $A$ is $B_{1}$-normalizable.

Proof. Induction on $\sigma_{1}(A)$. The proof is analogous to that of Th. 2.16. However, some modifications are required due to the $\stackrel{\circ}{P}_{i}$. We

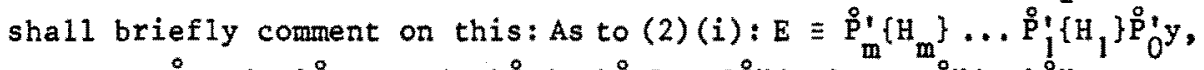

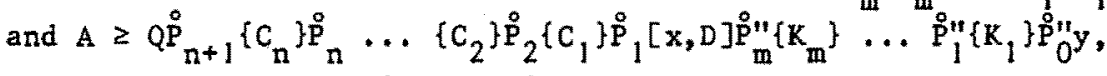
where the $K_{i}$ are obtained as in the proof of Th. 2.16 and where $Q P_{1}^{\prime \prime} \tau$ are the $\beta_{1}$-normal forms of $Q\left(x:=C_{1}\right) P_{i}^{0} \tau$. These can be obtained since either (1) if $x \notin \stackrel{P}{P}_{i}^{\prime}: \stackrel{P}{P}_{i}^{\prime \prime} \equiv \stackrel{P}{P}_{i}^{t}$, or (2) if $x \in \stackrel{P}{P}_{i}^{\prime}$ : $\sigma_{1}\left(Q\left\{C_{1}\right\}[x, D] P_{i}^{0} \tau\right) \equiv\left|\rho C_{1}\right| \leq \sigma(A)$ and $\left|P_{i}^{i} \tau\right|<|F|$ (apply the induc-

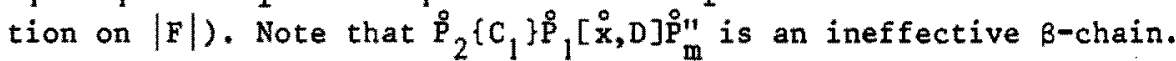
As to (2) (iii): $\operatorname{FrC}_{1}$ can be $\stackrel{\circ}{P}_{0}[x, M] N$, If $x \in N$, then the proof is similar to that of Th. 2.16. If $\mathrm{x} \notin \mathrm{N}$, we can take $[\mathrm{X}, \mathrm{M}]$ as part of an ineffective $\beta$-chain $\left\{K_{1}\right\} P_{0}^{\prime \prime} P_{0}^{\circ}[\stackrel{\circ}{x}, M]$, and look at the structure of $\mathrm{N}$ instead of that of $\mathrm{FrC}_{1}$.

This amounts to looking for the first "effective" abstractor in $N$. If there is such an abstractor and the obtained expression is not yet in $\beta_{1}$-normal form, induction is applicable as in Th. 2.16. If not, we have already obtained $\beta_{1}$-normal form. 
As to (2) (iv): $\mathrm{E} \equiv \stackrel{\circ}{\mathrm{P}}_{0}\left[\mathrm{y}, \mathrm{H}_{1}\right] \mathrm{H}_{2}$. If $\mathrm{y} \subset \mathrm{H}_{2}$ (so $\mathrm{y} \subset \mathrm{K}_{2}$ ), the proof is obvious. If not, look at $K_{2}$ instead of $E$, as in the previous case.

Theorem $3.10\left(B_{1}\right.$-normalization theorem $)$. If $A \in \Delta$ is p-normable, then $A$ is $B_{1}$-normalizable.

Proof. Induction on the length of proof of $A \in \Delta$, analogously to the proof of Th. 2.17. As to case (4) of this proof: the only case worth mentioning is $C^{\prime} \equiv \stackrel{8}{\mathrm{P}}[\mathrm{x}, \mathrm{D}] \mathrm{E}$. If $\mathrm{x} \subset \mathrm{E}$, then Th. 3.9 yields the desired result, and if $x \notin E$ we have already obtained $\beta_{1}$-normal form.

Theorem 3.11 ( $\beta_{1}$-nomalization theorem for $\left.\Lambda\right)$. If $A \in \Lambda$, then $A$ is $\beta_{1}$-normalizable.

Proof. Follows from Th. 2.7 and Th. 3.10 .

In fact we have proved that $A \in \Lambda$ is effectively $B_{1}$-normalizab1e.

Theorem 3.12. Let $A \in \Delta$ and $A \geq \geq_{B}^{\prime} B$. Then $|A| \leq|B|$.

Proof. Induction on the length of proof of $A \geq Z_{B}^{\prime} B$.

Definition 3.13. Let $A \in A$. We write $B_{1}$-nf $A$ for the $B_{1}$-normal form of A which we obtain from the effective computation as suggested by Th. 3,10 and used in Th. 3.11.

Note: this $\beta_{1}$-normal form is unique (Th. 3.3 ).

Definition 3.14. We call $\mathrm{K} \in \Delta$ strongly B-normalizable if there is an upper bound for the length $\ell$ of reduction sequences $k \equiv K_{1} \geq_{B}^{\prime}$ $K_{2} \geq_{B}^{\prime} \ldots \geq_{B}^{\prime} K_{\ell}$. Analogous $1 y$ we define the concepts strong $B_{1}-, B_{2}-$ or n-normalizability of $k$. 
Theorem 3.15 (strong $B_{2}$-normalization theorem for $\Delta$ ). If $A \in \Delta$, then $A$ is strongly $B_{2}$-normalizable.

Proof. Induction on $|A|$.

Definition 3.16. Let $A \in \Delta$, and let $A \equiv A_{1} \geq_{B_{2}}^{\prime} \quad A_{2} \geq_{B_{2}}^{\prime} \ldots \geq_{B_{2}}^{\prime} A_{p}$ be the longest possible sequence of single-step $\beta_{2}$-reductions beginning with $A$, Then $\theta_{2}(A)=p$.

Note that $\mathrm{p} \leq|\mathrm{A}|$.

Theorem 3.17. If $A \in \Lambda$ and $A z_{B_{1}}^{\prime} B$, then $\theta_{2}\left(B_{1}-n f A\right)=\theta_{2}\left(B_{1}-n f B\right)$. Proof. Follows from Th. 3.3.

Theorem 3.18. If $A \geq_{B_{1}}^{\prime} B$, then $\theta_{2}(A)<\theta_{2}(B)$.

Proof. Induction on the length of proof of $A \geq_{B_{1}}^{\prime} B$. The only interesting case is $A \equiv Q\{C\} \hat{P}[x, D] E \geq_{B}^{\prime}, Q\{C\} \hat{P}[\hat{x}, D](x:=C) E \equiv B$, where, indeed, we have at least one single-step $\beta_{2}$-reduction more on the right hand side. The rest of the proof is easy.

Corollary 3.19. If $A \in \Lambda$, then $\theta_{2}(A) \leq \theta_{2}\left(B_{1}-\right.$ nf $\left.A\right)$.

Theorem 3.20 (strong $B_{1}$-normalization theorem for $A$ ). If $A \in \Lambda$, then $A$ is strongly $B_{1}$-normalizable.

Proof. Follows from th. 3.17, Th. 3.18 and Cor. 3.19.

Definition 3.21. Let $A \in \Lambda$, and let $A \equiv A_{1} \geq_{B_{1}}^{\prime} A_{2} \geq_{B_{1}}^{\prime} \ldots \geq_{B_{1}}^{\prime} A_{p}$ be the longest possible sequence of single-step $\beta_{1}$-reductions beginning with $A$. Then $\theta_{1}(A)=p$. 
Theorem 3.22. Let $A \in A$, let $A$ be in $B_{1}$-normal form and let $A \geq_{B_{2}} B$. Then $B$ is also in $B_{1}$-normal form.

Proof. If $B$ were not in $B_{1}$-normal form, then $B \geq B_{1}^{\prime} C$ for some $C$. In that case there would be a reduction $A \geq_{B_{1}}^{\prime} B^{\prime} \geq_{B_{2}} C$ according to Th. II,6.17. Contradiction.

Theorem 3.23. If $A \in A$, then there is an upper bound for the length $\ell$ of reduction sequences $A \equiv A_{1} \geq^{\prime} A_{2} \geq ' \ldots \geq^{\prime} A_{\ell}$, where each $A_{i} \geq A_{i+1}$ is a single-step $B_{1}$ - or $\beta_{2}$-reduction.

Proof. Induction on $\theta_{1}(A)$. If $\theta_{1}(A)=0$, then $A$ is in $B_{1}$-normal form. If we can apply $B_{2}$-reductions on $A$ such that $A \geq \sum_{2}^{n} B(n \geq 1)$, then $B$ is also in $\beta_{1}$-normal form (Th. 3.22). The number of possible single-step $B_{2}$-reductions applicable is finite $\left(\leq \theta_{2}(A) ; c f\right.$. Th. 3.15).

So let $\theta_{1}(A)=p>0$, and assume that the theorem holds for all $\mathrm{K}$ with $\theta_{1}(K)<\mathrm{p}$. Let $A \geq D$ be a reduction sequence consisting of single-step $B_{1}$ - and $B_{2}$-reductions. If no $\beta_{1}$-reductions occur in the reduction sequence, the length of the reduction sequence can be at most $\theta_{2}(A)$. Else, let $A \geq D$ be $A \geq_{B_{2}}^{n} B \geq_{B_{1}}^{\prime} C \geq D$. Then by Th. II.6.17 there is also a reduction sequence $A \geq_{B_{1}}^{\prime} B^{\prime} \geq_{B_{2}} C \geq D$. Each $B^{\prime \prime}$ such that $A \geq_{B_{1}}^{\prime} B^{\prime \prime}$ has by induction (since $\theta_{1}\left(B^{\prime \prime}\right)<\theta_{1}(A)$ ) an upper bound for the length of reduction sequences $B^{\prime \prime} \geq^{\prime} \ldots \geq^{\prime} E$ in which each single-step reduction is either a $\beta_{1}{ }^{-}$or a $\beta_{2}$-reduction. Let $m$ be the maximum of these upper bounds. Then the length of the reduction sequence $B^{\prime} \geq{ }_{B_{2}} C \geq D$, hence of $C \geq D$, cannot be more than $m$. It follows that the length of any reduction sequence $A \geq D$ can be at most $\theta_{2}(A)+m+1$.

Theorem 3.24 (strong B-normalization theorem for $\Lambda$ ). If $A \in \Lambda$, then $A$ is strongly $\beta$-normalizable. 
Proof. Each B-reduction sequence of $A$ can be decomposed into singlestep $B_{1}{ }^{-}$and $B_{2}$-reductions by Th. II.6.15. So Th. 3.23 yields the desired result.

Definition 3.25. Let $A \in A$, and let $A \equiv A_{1} \geq_{\beta}^{\prime} A_{2} \geq_{B}^{\prime} \ldots \geq_{\beta}^{\prime} A_{p}$ be the longest possible sequence of single-step $\beta$-reductions beginning with $A$. Then $\theta(A)=p$.

Theorem 3.26 (strong n-normalization theorem for $\Delta$ ). If $A \in \Delta$, then $A$ is strongly n-normalizable.

Proof. Induction on $|A|$.

Definition 3.27. We call $\mathrm{K} \in \Delta$ strongly normalizable if there is an upper bound for the length $\ell$ of reduction sequences $K \equiv K_{1} \geq K_{2} \geq '$ $\ldots \geq^{\prime} k_{2}$ where each reduction $k_{i} \geq^{\prime} k_{i+1}$ is a single-step $B-$ or $n^{-}$ reduction.

Theorem 3.28 (strong normatization theorem for $\Lambda$ ). If $A \in \Lambda$, then $A$ is strongly normalizable.

Proof. Induction on $\Theta(A)$. The proof is similar to that of Th, 3.23. Use Th. 3.26 instead of Th. 3.22, and instead of Th. II.6.17, use the theorem: If $K \in \Delta, K \geq_{\eta} L \geq_{B}^{\prime} M$, then $K \geq_{B}^{\prime} L^{\prime} \geq M$. The latter theorem is easy to prove, since each reduction $A \geq_{\eta}^{\prime} B \geq_{B}^{\prime} C$ can be replaced either by a reduction $A \geq_{B}^{\prime} B^{\prime} \geq_{\eta}^{x} C$ (where $r \geq 0$ ) or by a reduction $A z_{B}^{\prime} B^{\prime} z_{B}^{\prime} C$ (see p. 65; see also Th. 7.25). 


\section{REFERENCES}

[1] BARENDREGT, H.P., Some extensional term models for combinatory logics and $\lambda$-calculi, Thesis, Utrecht, 1971.

[2] CHURCH, A., A set of postulates for the foundation of logic, Ann. of Math. (2) 33, p.346-366 (1932) and 34, p. 839-864 (1933).

[3] CURRY, H.B. and FEYS, R., Combinatory Logic, Vol. I, North Holland Publishing Company, Amsterdam, 1958.

[4] DE BRUIJN, N.G., The mathematical language AUTOMATH, its usage, and some of its extensions, Symposium on Automatic Demonstration (Versailles December 1968), Lecture Notes in Mathematics, Vo1. 125, p. 29-61, Springer-Verlag, Berlin, 1970.

[5] DE BRUIJN, N.G., Automath, a language for mathematics, THEreport 68-WSK-05, Technological University, Eindhoven, 1968.

[6] DE BRUIJN, N.G., Automath, a language for mathematics; notes (prepared by B. Fawcett) of a series of lectures in the Sếminaire de Mathématiques Supérieures, Université de Montreal, 1971 .

[7] DE BRUIJN, N.G., AUT-SL, a single line version of AUTOMATH, Technological University Eindhoven, Internal Report, Notitie 22, 1971 .

[8] DE BRUIJN, N.G., Lambda calculus notation with nameless dummies, a tool for automatic formula manipulation, with application to the Church-Rosser theorem, Indag. Math., 34, No. 5, 1972.

[9] GIRARD, J.Y., Une extension de l'interpretation de Gödel à l'analyse, et son application à l'élimination des coupures dans l'analyse et la théorie des types, in: Proc. 2nd 
Scandinavian Logic Symp. (editor Fenstad), North-Holland Publishing Company, Ansterdam, 1970.

[10] HOWARD, W.A., The formulae-as-types notion of construction, mimeographed, 1969.

[11] LAUCHLI, H., An abstract notion of realizability for which intuitionistic predicate calculus is complete, in: Intuitionism and proof theory, Proc. Summer. Conference at Buffalo 1968 (editors Kino et.al), North-Holland Pubishing Company, Amsterdam, 1970.

[12] MARTIN-LOF, P., An intuitionistic theory of types, unpublished, 1972 .

[13] NEDERPELT, R.P., Lambda-Automath, Technological University Eindhoven, Internal Report, Notitie 17, 1971.

[14] NEDERPELT, R.P., Lambda-Automath II, Technologica1 University Eindhoven, Internal Report, Notitie 25, 1971.

[15] NEDERPELT, R.P., The closure theorem in $\lambda$-typed $\lambda$-calculus, Technological University Eindhoven, Internal Report, Notitie $22,1972$.

[16] NEDERPELT, R.P., Strong normalisation in a $\lambda$-calculus with $\lambda$ expressions as types, Technological University Eindhoven, Internal Report, Notitie 18, 1972.

[17] PRAWITZ, D., Natural deduction, a proof-theoretical study, Almquist and Wikse11, Stockholm, 1965.

[18] PRAWITZ, D., Ideas and results in proof theory, in: Proc. 2nd Scandinavian Logic Symp., North-Holland Publishing Company, Amsterdam, 1971.

[19] SANCHIS, L.E., Functionals defined by recursion, Notre Dame journal of formal logic 8, p. 161-174, 1967.

[20] SCHOENEIELD, J.R., Mathematical logic, Addison-Wesley Publishing Company, Reading, Massachusetts, 1967. 
[21] TAIT, W.W., Intentional interpretations of functionals of finite type I, The journal of symbolic logic 32 , p. $198-212,1967$.

[22] VAN BENTHEM JUTTING, L.S., On normal forms in Automath, Technological University Eindhoven, Internal Report, Notitie 24, 1971 .

[23] KREISEL, G., Five notes on the application of proof theory to computer science, Techn. report no. 182, Institute for mathematical studies in the social sciences, Stanford University, Stanford, Califurnia, 1972.

[24] SCHULTE MÖNTIN, H., Yet another proof of the Church-Rosser theorem, unpublished, 1973. 


\section{SAMENVATTING}

Aan de lambda-calculus ligt een formele generalisatie van de wiskundige begrippen functie en functietoepassing ten grondslag. In een getypeerde lambda-calculus worden de expressies uit de calculus van typen voorzien om de interpreteerbaarheid in gangbare wiskundige termen te vergemakkelijken. De toepasbaarheid van een functie op een argument wordt in een getypeerde lambda-calculus vaak op een natuur1ijke manier beperkt in overeenstemming met de type toekenning (de toepasbaarheidsvoomwarade).

In dit proefschrift worden twee systemen van getypeerde lambdacalculus onderzocht, die $\Delta$ en $\Lambda$ worden genoemd. Het systeem $\Lambda$ is een deelsysteem van $\Delta$. In $\Delta$ wordt de toepasbaarheidsvoorwaarde nog niet gesteld, in $\Lambda$ gebeurt dit wel. De typen in de systemen $\Delta$ en $\Lambda$ zijn algemeen in die zin, dat ze dezelfde structuur hebben als de expressies. Het systeem $\Lambda$ is voortgekomen uit de wiskundige taal Automath.

Hoofdstuk I van dit proefschrift geeft een inleiding in de lambda-calculus en beschrijft de typetoekenning. Voorts wordt het verband met Automath besproken. Een uitgebreide samenvatting $v$ an het proefschrift besluit dit hoofdstuk.

In Hoofdstuk II wordt het systeem $\Delta$ geintroduceerd en besproken De uit de lambda-calculus bekende relaties worden ook in het systeem $\Delta$ ingevoerd. Daarnaast worden andere relaties gedefinieerd en onderzocht als voorbereiding op Hoofdstuk III.

In Hoofdstuk III wordt het systeem $\Lambda$ ingevoerd. Er wordt aangetoond dat de toepasbaarheidsvoorwaarde voldoende is om voor alle expressies in $\Lambda$ normaliseerbaarheid te bewerkstelligen: we noemen een expressie normaliseerbaar als men functietoepassingen binnen de expressie in eendusdanige volgorde kan uitvoeren, dat er na een eindig aantal stappen een expressie ontstat warin geen functietoepassing meer mogelijk is. In het laatste geval zegt men dat de normaalvorm is bereikt. 
In het systeem $\Lambda$ blijkt zelfs sterke normaliseerbaarheid te gelden: iedere volgorde van functietoepassingen moet in een eindig aantal stappen tot de normalvorm leiden. Dit wordt eveneens bewezen in Hoofdstuk III, met gebruikmaking van de hierboven genoemde (gewone) normaliseerbaarheid. 


\section{CURRICULUM VITAE}

De schrijver van dit proefschrift werd op 19 juli 1942 geboren in Den Haag. Van 1953 tot 1959 doorliep hij de gymnasiumafdeling van het Nederlands Lyceum in deze stad. Daarna begon hij een studie aan de Rijksuniversiteit te Leiden. Na een studie-onderbreking legde hij hier in 1965 het candidaatsexamen sterre- en wiskunde af en in juni 1969, cum laude, het doctoraalexamen wiskunde met bijvak Russisch. In het studiejaar 1966-1967 was hij als assistent werkzaam bij de rekenafdeling van het Mathematisch Centrum te Amsterdam. Sinds $z i j n$ afstuderen is hij als wetenschappelijk medewerker werkzaam bij de Onderafdeling der Wiskunde van de Technische Hogeschoo1 te Eindhoven, met. een wetenschappelijke taak in het onderzoek van de wiskundige taal Automath, onder leiding van Prof.dr. N.G. de Bruijn, en met een onderwijstaak in het basisonderwijs.

Address of the author:

Technological University, Dept. of Mathematics, Eindhoven, The Netherlands. 
1. Noem een rechthoek met afmetingen $1 \times p$ een strook van lengte $p$. Definieer een $k$-set als een verzameling stroken $\left\{\sigma_{1}, \sigma_{2}, \ldots, \sigma_{k}\right\}$, waarbij $\sigma_{i}$ een strook van lengte $i$ is. Men kan trachten om bij zekere $k$ met alle stroken uit $n$ k-sets een rechthoek op te vullen; hierbij mogen de stroken in de lengte- en in de breedterichting worden gelegd. Als er binnen een zo gevulde rechthoek geen rechte $1 \mathrm{ijn}$ is die twee overstaande zijden verbindt, zeggen we dat met. de $\mathrm{n}$ k-sets een hechte rechthoek is geconstrueerd.

Als bij gegeven $\mathrm{k}$ met $\mathrm{n}$ k-sets een hechte rechthoek kan worden geconstrueerd, terwijl dit met minder dan $n$ k-sets niet mogelijk is, definiëren we $m_{k}=n$. Er geldt:

(1) $\mathrm{m}_{\mathrm{k}}$ bestaat voor alle $\mathrm{k}$ en $\mathrm{m}_{\mathrm{k}} \leq 2$ als $\mathrm{k} \neq 2$.

(2) $\mathrm{m}_{2}=8$.

(3) $k=14$ is het kleinste getal (afgezien van 1) waarvoor $\mathrm{m}_{\mathrm{k}}=1$.

(4) Voor oneindig veel waarden van $k$ geldt $m_{k}=1$.

2. Zij een verzameling $\mathrm{V}$ van termen gegeven door de volgende inductieve definitie:

(1) ( ) is een term van $V$.

(2) Als $A$ en $B$ termen van $V z i j n$, dan is ( $A B$ ) een term van $V$.

Definieer de lengte $|A|$ van een term A inductief door:

(1) $|()|=1$.

(2) $|(A B)|=|A|+|B|$.

$\mathrm{Zij} \sim$ de equivalentierelatie op $\mathrm{V}$ gegenereerd door:

(1) $((\mathrm{AB}) \mathrm{C}) \sim((\mathrm{AC}) \mathrm{B})$.

(2) Als $A \sim B$ en $C \sim D$, dan $(A C) \sim$ (BD).

(A, B, C en D zijn termen van V.)

Termen uit een equivalentieklasse $K$ hebben gelijke lengte; noteer deze lengte als $\ell(K)$. Er is nu een natuurlijke één-éénduidige afbeelding $T$ van de verzameling van equivalentieklassen $k$ op de verzameling van eindige "rooted trees", met de eigenschap dat voor iedere $K \ell(K)$ gelijk:is aan het aantal knooppunten van $\mathrm{T}(\mathrm{K})$.

(Opmerking: Theorem 2.2 uit [1] is hiervan een direct gevolg.)

[1] F. Göbel en R.P. Nederpelt, The number of numerical outcomes of iterated powers, Amer. Math. Monthly, Vol. 78, No. 10, december 1971. 
3. We nemen aan dat op elk kruispunt van de stad $X$. precies $v$ wegen samenkomen en dat elk huizenblok wordt begrensd door tenminste drie straten; verbindingswegen van $x$, met de rest van de wereld bestaan niet. Voorts heeft de stad een circuit van wegen met een bijzondere eigenschap: als men dit circuit eenmaal rondrijdt, blijkt het dat men bij opvolgende kruispunten afwisselend de meest linkse en de meest rechtse afslag neemt, terwiji geen kruispunt tweemal wordt gepasseerd. Het circuit telt een even aantal (2k) kruispunten.

Over het aantal kruispunten $f_{v}(2 k)$ dat $X$. tenminste moet hebben kunnen we het volgende zeggen:

(1) $f_{3}(4 k)=4 k, f_{3}(4 k+2)=4 k+4 \quad(k=1,2, \ldots)$.

(2) $\mathrm{f}_{4}(4)=9, \mathrm{f}_{4}(2 \mathrm{k})=2 \mathrm{k} \quad(\mathrm{k}=3,4, \ldots)$.

(3) Als $v=5$ kan een bovengrens $b(2 k)$ worden aangegeven voor $f_{v}(2 k)$, die voor voldoend grote $k$ voldoet aan $b(2 k) \leq 2 k+2\left[\frac{2 k}{10}\right]+4$.

4. De laatste paragraaf ( $\$ 3.5 .5$ ) van Wegners beschrijving van de lambda-calculus in [2] bevat een aantal onnauwkeurigheden.

.[2] P. Wegner, Programming languages, information structures, and machine organization, McGraw-Hi11 Book Company, New York, 1968.

5. Het zou goed zijn als men bij de vernieuwing van het wiskunde-onderwijs op middelbare school niveau ook aandacht zou schenken aan de combinatoriek. Wiskundeleraren kunnen uit [3] een goed inzicht krijgen in de achtergronden van dit vak.

[3] G. Bergman en K.D. Fryer, Introduction to combinatorics, Academic Press, Inc., New York, 1972.

6. Een notatie van functies die aansluit bij de lambda-notatie van A. Church verdient uit didactisch en uit wetenschappelijk oogpunt de voorkeur boven de traditionele notatie. Ook in de theorie van de programmeertalen kan de lambda-notatie zijn nut bewijzen. 